\title{
Abstracts for the 44th Human Genetics Society of Australasia Annual Scientific Meeting, 14-17 August 2021
}

\section{Poster Presentations}

\section{CLINICAL GENETICS}

\section{ACT Genetic Service Approach to 'Rebooting' Cancer Genetic Testing Wait Times After COVID-19}

Kezia Bates, Linda Warwick, Amanda Engel, Belinda Dopita, Stephanie Badman, Grace Phillips and Jennifer Rigby

Canberra Health Services, Canberra, ACT, Australia

Background: The ACT Genetic Service acquired funding under Operation Reboot, a \$3.5-million initiative developed by Canberra Health Services to address increased wait times due to COVID-19. This funding was used to employ an Associate Genetic Counselor for 8 weeks with the objectives to: (1) see $100 \%$ of CAT1 patients in 30 days, (2) see $90 \%$ of CAT2 patients in 90 days, and (3) reduce CAT3 referrals by $40 \%$ of June 2020 numbers. Aims: To analyze the effectiveness of our approach to Operation Reboot. Methods: Waiting times for cancer genetic testing at the ACT Genetic Service were extracted from November 2019 to March 2021, and descriptive statistics were used to analyze the data. For the purpose of analysis, 'COVID' refers to April 2020-November 2020, 'OpReboot' refers to December 2020-January 2021 and 'Post-OpReboot' refers to February 2021-March 2021. Results: The average number of patients seen per month increased by 144\% during Operation Reboot (COVID: 10 patients; OpReboot: 26 patients). The intervention decreased average CAT1 and CAT2 wait times by 63\% (COVID: 2.4 months; PostOpReboot: 0.9 months) and 28\%, respectively (COVID: 2 months; Post OpReboot: 1.5 months). The CAT3 average wait times increased by $53 \%$ of June 2020 numbers from 8.0 to 12.2 months in the postOpReboot period. Conclusion: The objectives for both CAT1 and CAT2 referrals were met as a result of Operation Reboot. The CAT3 objective was not met although this intervention may allow for increased opportunity for this to be addressed in future.

\section{Heterogeneity in How Women Value Risk-Stratified Breast Screening}

Jack Wheeler ${ }^{1,2}$, Louise Keogh ${ }^{2}$, Maria Sierra ${ }^{3}$, Lisa Devereaux ${ }^{4}$, Katherine Jones ${ }^{1}$, Maarten IJzerman ${ }^{2,6,7}$ and Alison Trainer ${ }^{1,5}$

${ }^{1}$ Parkville Familial Cancer Centre, Peter MacCallum Cancer Centre, Melbourne, VIC, Australia, ${ }^{2}$ Centre for Health Equity, School of Population and Global Health, The University of Melbourne, VIC, Australia, ${ }^{3}$ Miller School of Medicine, University of Miami, Miami, FL, USA, ${ }^{4}$ Lifepool Study, Peter MacCallum Cancer Centre, Melbourne, VIC, Australia, ${ }^{5}$ Department of Medicine, The University of Melbourne, VIC, Australia, ${ }^{6}$ Centre for Cancer Research, University of Melbourne, VIC, Australia and ${ }^{7}$ Department of Cancer Research, Peter MacCallum Cancer Centre, Melbourne, VIC, Australia

Background: Risk-stratified screening has potential to improve benefit-to-harm ratios of population breast cancer (BC) screening programs. We hypothesize a person-centered approach to implementation will optimize participation rates. Aim: To explore what women would value when deciding to participate in a risk-stratified breast screening program by having a personal BC risk assessment, and the relative impact of each factor on their decision. Methods: Women across the $\mathrm{BC}$ risk spectrum were recruited through the LifePool study and Parkville Familial Cancer Centre. Multicriteria decision analysis was used to determine the relative weights and level preferences of eight criteria women reported would influence their decision to participate in a personal BC risk assessment. Latent preference heterogeneity was explored through cluster analysis. Results: Of 363 women surveyed, 95\% would accept a personal BC risk assessment. The two criteria most highly valued by participants related to program access, 'accessing information' and 'testing process', both of which significantly influenced participation $(p<.001) .73 \%$ preferred information by letter/ online. Storage of genomic data had less impact than expected $(p<.001)$. Four latent preference-based subgroups were identified. Women in the largest subgroup focussed on program access, with membership predicted by low educational attainment. Higher relative perceived breast cancer risk predicted membership of the smallest subgroup who valued test parameters: 'scope of test' and 'test specificity'. Discussion/Conclusion: These findings suggest that a population-based $\mathrm{BC}$ risk assessment framework may be socially acceptable. When implementing a breast screening program tailored to personal risk, these findings suggest population acceptance and participation can be maximized through tailoring implementation to population preference-based subgroups.

\section{Expanding the POT1 Tumor Predisposition Syndrome}

Vaishnavi Nathan ${ }^{1,2}$, Jane M. Palmer ${ }^{1}$, Peter A. Johansson ${ }^{1}$, Hayley R. Hamilton ${ }^{1}$, Madeleine Howlie ${ }^{1}$, Sunil K. Warrier ${ }^{3}$, William Glasson ${ }^{3}$, Lindsay A. McGrath ${ }^{3}$, Vivian F. S. Kahl ${ }^{4}$, Raja S. Vasireddy ${ }^{5}$, Hilda A. Pickett ${ }^{4}$, Kelly M. Brooks ${ }^{1}$, Antonia L. Pritchard ${ }^{1,6}$ and Nicholas K. Hayward ${ }^{1}$

${ }^{1}$ QIMR Berghofer Medical Research Institute, Brisbane, QLD, Australia, ${ }^{2}$ University of Queensland, Brisbane, QLD, Australia, ${ }^{3}$ Queensland Ocular Oncology Services, Brisbane, QLD, Australia, ${ }^{4}$ Children's Medical Research Institute, University of Sydney, Sydney, NSW, Australia, ${ }^{5}$ Children's Hospital at Westmead, Sydney Children's Hospital Network, Sydney, NSW, Australia and ' University of the Highlands and Islands, Inverness, Scotland, UK

Background: 'Protection of telomeres 1' (POT1) binds to telomeres to shield them from excessive degradation or lengthening. Pathogenic variants in POT1 result in a tumor predisposition syndrome (POT1TPDS) which includes susceptibility to angiosarcoma, chronic lymphocytic leukemia, colorectal cancer, cutaneous melanoma 
(CM) and glioma. Here, we expand the spectrum of cancers to include uveal melanoma (UM) and a range of hematological malignancies. Aim: The aim of this study was to identify the genetic cause of disease predisposition in high-risk CM and UM families, and to conduct functional evaluation of candidate susceptibility variants. Methods: Patients $(n=461)$ were recruited from Queensland, and DNA samples were collected to be sent for next generation sequencing. Telomere length assays were done for functional characterization of POT1 variants. Results: Three variants, c.281_282del, c.1458_1459del, and c.670G $>$ A in POT1 were identified. The two loss-of-function variant carriers had both CM and UM, and functional characterization showed carriers had longer telomeres, compared to age-matched healthy controls. Currently classified as a VUS in ClinVar, missense variant c.670G $>A$ was identified in a family with $\mathrm{CM}$ and hematological malignancies not yet associated with the POT1-TPDS, including follicular lymphoma and chronic myeloid leukemia. Functional assessment has previously shown c.670G $>$ A to disrupt POT1 binding, leading to longer, more fragile telomeres. Conclusion: The genetic basis of cancer susceptibility in three families from our study was found to be caused by pathogenic variants in POT1. Our findings indicate that pathogenic variants in POT1 predispose to a wide range of tumor types, having broader implications for genetic testing, screening, and counseling recommendations.

\section{Not So Fast: Why Somatic Testing in Cancer Patients Doesn't Tell the Whole Story}

Nandor Roczo, Sarah M. Nielsen, Kingshuk Das, Nhu Ngo, Edward D. Esplin and Robert L. Nussbaum

Invitae, San Francisco, CA, USA

Background: Both germline and somatic genetic testing are wellestablished tools for precision medicine in cancer patients. Sequencing of a tumor detects somatic variants which can be used to inform therapy and can also detect germline variants, but with important limitations. As such, these tests can fail to report clinically actionable germline events. Methods: We retrospectively analyzed a cohort of 2023 cancer patients with previous tumor sequencing results who were receiving germline testing for cancer predisposition genes. Indications for germline testing included: tumor findings of potential germline origin, treatment guidance or surgical planning, and personal/family history. High and moderate risk pathogenic and likely pathogenic germline variants (PGVs) were analyzed. Results: Overall, $30.5 \%(n=617)$ of patients harbored a PGV, most of which could have treatment implications. PGVs were prevalent across cancer types, genes, and patient age. Importantly, $8.1 \%$ of PGVs (50) were not reported by tumor sequencing as either somatic or germline findings. Among patients with PGVs, 11.2\% (69) had their PGVs identified only after presenting with a second, possibly preventable, primary malignancy. Variants were often observed in patients with cancers not strongly associated with their germline findings, such as lung cancer and CNS tumors. Conclusions: As a follow-up to tumor sequencing, germline tests can inform clinical management decisions. Current guidelines and tumor testing approaches capture many, but not all, of these germline findings, reinforcing the utility of both expanded germline reflex testing, as well as germline analysis independent of tumor sequencing in appropriate patients.

\section{Carriers' Experience and Attitudes Towards the Australian Jewish Brca2/2 Community Screening Program}

Natalie Nowak ${ }^{1,2}$, Nicole Cousens ${ }^{3}$, Martin Delatycki ${ }^{4,6}$, Jane Tiller ${ }^{6}$, Chris Jacobs $^{2}$ and Lesley Andrews ${ }^{3}$

${ }^{1}$ Agnes Ginges Centre for Molecular Cardiology, Centenary Institute, Sydney, NSW, Australia, ${ }^{2}$ Graduate School of Health, University of Technology Sydney, Sydney, NSW, Australia, ${ }^{3}$ Hereditary Cancer Clinic, Prince of Wales Hospital, Sydney, NSW, Australia, ${ }^{4}$ Bruce Lefroy Centre, Murdoch Children's Research Institute, Royal Children's Hospital, Melbourne, VIC, Australia, ${ }^{5}$ School of Public Health, Monash University, Melbourne, VIC, Australia and ${ }^{6}$ Victorian Clinical Genetic Services, Murdoch Children's Research Institute, Melbourne, VIC, Australia

Background: Individuals of Jewish Ancestry have approximately a 1 in 40 chance of testing positive for three Jewish Founder Mutations in $B R C a 2$ and $B R C A 2$. An Australian first Jewish BRCa2/2 screening program (JeneScreen) was offered in two Australian metropolitan cities. The cities used different pretest information delivery and consent methods; one via a website (online) and the other via community seminars (community). This substudy aimed to explore the experiences of participants who tested positive through JeneScreen. Methods: BRCa2/2 positive JeneScreen participants were invited to partake in the substudy via email. Semistructured telephone interviews were conducted to explore experiences of the pretest method, receiving results, and participating in the program. Interviews were analyzed using an inductive thematic analysis. High coding concordance was achieved across three coders. Results: Three online and nine community participants were interviewed, 11/12 were analyzed, identifying three key themes: (1) Appreciation of JeneScreen reflects participant's feelings about the screening program. (2) Feeling supported relates to the participants' experiences throughout the JeneScreen genetic testing experience. (3) 'JeneScreen, it is an easy process' encompasses the participants' perceived benefits of population screening and the use of streamlined methods. All participants were supportive of a $B R C a 2 / 2$ screening program and most preferred the pre-test method they received. Conclusion: Both online and community participants expressed high satisfaction with the screening program. This study suggests that a website and community seminars are suitable replacements for face-to-face pre-test counseling in a $B R C a 2 / 2$ Jewish founder mutation screening program.

\section{A Germline Novel Truncating MECOM Variant in a Patient with a Family History of Thrombocytopenia}

Lesley Rawlings ${ }^{1}$, Cassandra Vakulin ${ }^{1}$, Lucas Dejong ${ }^{1}$, Rema Fathi ${ }^{1}$, Anna L. Brown ${ }^{1}$, Simon McRae ${ }^{2}$, Nicholas Myles ${ }^{2}$, Devendra Hiwase ${ }^{2}$, Yvonne Brennan ${ }^{2}$ and Hamish S. Scott ${ }^{1}$

${ }^{1}$ Department of Genetics and Molecular Pathology, SA Pathology, Adelaide, SA, Australia and ${ }^{2}$ Department of Hematology, SA Pathology and Royal Adelaide Hospital, Adelaide, SA, Australia.

Background: Pathogenic variants in the MECOM gene are associated with amegakaryocytic thrombocytopenia with/without radioulnar synostosis (OMIM:616738). Aim: Most cases reported to date have required transplants at relatively young ages. We present a family with mild thrombocytopenia without radioulnar synostosis with a likely pathogenic variant detected in the MECOM gene, who have not required a transplant. Case Description: A 54-year-old male, diagnosed at birth with thrombocytopenia, with spontaneous 
improvement at 12 months of age. At age 11, he was again diagnosed with aplastic anemia that has persisted. His father had thrombocytopenia, a sister diagnosed at age 59 with mild intermittent thrombocytopenia and neutropenia, a brother who died aged 8 years with aplastic anemia and a brother who was stillborn. Method and Results: Next-generation sequencing was performed on the proband's germline (hair) DNA sample and his sister's blood-derived DNA sample with analysis restricted to genes within a comprehensive bone marrow failure panel. Analysis detected a likely pathogenic truncating mutation in the MECOM gene (NM_004991.3): c.816dupT resulting in p.(Pro273Serfs $\left.{ }^{\star} 2\right)$. Conclusion: This earlytruncating MECOM mutation, located in the first zinc finger, is likely a complete loss of functional allele. MECOM mutations that cause thrombocytopenia and bone marrow failure with radioulnar synostosis described to date are missense mutations in the second c-terminal zinc finger. Stillbirth seems to be a feature of MECOM with postmortem analysis of several fetuses in affected families showing hypocellular BM. As well as stillbirth, this family is among the mildest and oldest mutant MECOM patients described to date.

\section{Clonal Hematopoiesis as a Natural Functional Assay of TP53 Germline Variant Pathogenicity: Updated Results From Two Laboratories}

Cristina Fortuno ${ }^{1}$, Tina Pesaran², Jill Dolinsky², Kelly McGoldrick², Lily Hoang ${ }^{2}$, Paul A. James ${ }^{3}$ and Amanda B. Spurdle ${ }^{1}$

${ }^{1}$ Genetics and Computational Division, QIMR Berghofer Medical Research Institute, Brisbane, QLD, Australia, ${ }^{2}$ Ambry Genetics, Aliso Viejo, CA, USA and ${ }^{3}$ Parkville Familial Cancer Centre, Peter MacCallum Cancer Centre and Royal Melbourne Hospital, Melbourne, VIC, Australia

Approximately $30 \%$ of TP53 variants identified in the blood of patients undergoing genetic testing for hereditary cancer are suspected to have somatic origin, mostly due to clonal hematopoiesis $(\mathrm{CH})$, and this proportion increases with lower variant allele fraction (VAF). Positive selection has been proposed to be a mechanism driving $\mathrm{CH}$, with somatic variants providing fitness advantage to a subset of blood cells. For this reason, we hypothesized that TP53 variants with low VAF are pathogenic drivers of $\mathrm{CH}$. If true, low VAF status could be used a positive predictor of TP53 variant pathogenicity. We used VAF data from 1100 pathogenic variants and 65291 benign variants from Ambry Genetics, in addition to 355 pathogenic variants from Peter MacCallum Cancer Centre. Variants present in the homozygous state or in individuals affected with a hematological malignancy had been excluded. VAF distribution was strikingly different between the groups: benign variants showed an expected normal distribution around 50:50 ratio, while pathogenic variants showed a bimodal distribution. We compared the proportion of pathogenic and benign variants observed in different VAF bins, and determined that VAF $<30 \%$ provided strong evidence towards pathogenicity (likelihood ratio $>100$ ). We found that this evidence type could be applied to $25 \%$ of TP53 germline variants of uncertain significance. This data confirms that $\mathrm{CH}$ is a distinctive characteristic of pathogenic TP53 variants, and provides a new interpretation of this phenomenon. It can be used as a natural functional assay to assist with the interpretation of TP53 germline variants.

\section{The Impact of Variants at Branchpoint Splicing Elements in Cancer Genes}

Daffodil M. Canson ${ }^{1,2}$, Troy Dumenil ${ }^{1}$, Michael T. Parsons ${ }^{1}$, Tracy A. O'Mara ${ }^{1}$, Aimee L. Davidson ${ }^{1,2}$, Satomi Okano ${ }^{1}$, Bethany Signal ${ }^{3,4}$, Tim R. Mercer $^{3,4,5}$, Dylan M. Glubb and Amanda B. Spurdle ${ }^{1,2}$

${ }^{1}$ QIMR Berghofer Medical Research Institute, Brisbane, QLD, Australia, , ${ }^{2}$ Faculty of Medicine, The University of Queensland, Brisbane, QLD, Australia, ${ }^{3}$ Genomics and Epigenetics, Garvan Institute of Medical Research, Sydney, NSW, Australia, ${ }^{4}$ St Vincent's Clinical School, University of New South Wales, Sydney, NSW, Australia and ${ }^{5}$ Altius Institute for Biomedical Sciences, Seattle, WA, USA

Background: Branchpoint elements are required for intron removal, and variations in these elements can result in aberrant splicing. Aim: We aimed to assess the value of branchpoint annotations generated from recent large-scale studies to select branchpointabrogating variants, using hereditary cancer genes as model. Methods: We identified branchpoint elements in 119 genes associated with hereditary cancer from three genomewide experimentally inferred and two predicted branchpoint datasets. We then identified variants from public databases that occur within branchpoint elements. We compared conservation, unique variant observations, and population frequencies at different nucleotides within branchpoint motifs. Finally, selected minigene assays were performed to assess the functional impact of variants in branchpoint elements within mismatch repair genes. Results: We annotated 4,973 branchpoints (3,012 experimentally inferred) in hereditary cancer genes. There was poor overlap between predicted and experimentally inferred branchpoints. We found 6,494 single nucleotide variants were located in experimentally inferred or predicted branchpoint motifs. Our analysis of cancer genes suggested that variants at $2 \mathrm{nt},-1 \mathrm{nt}$, and branchpoint positions in experimentally inferred canonical motifs are more likely to be clinically relevant. Minigene assay data showed the -2 nt to be more important to branchpoint motif integrity, but also demonstrated fluidity in branchpoint usage. Conclusion: Data from cancer gene analysis suggests that there will be few high-risk alleles that severely impact function via branchpoint abrogation. Results inform a general scheme to prioritize branchpoint motif variants for further study.

\section{Exploring the Implementation of Comprehensive Genomic Breast Cancer Risk Assessments}

Maria Sierra', Jack Wheeler2'3, Alison Trainer2'4 and Louise Keogh3'5

${ }^{1}$ Miller School of Medicine, University of Miami, Miami, FL, USA, ${ }^{2}$ Parkville Familial Cancer Centre, Peter MacCallum Cancer Centre, Melbourne, VIC, Australia, ${ }^{3}$ Centre for Health Equity, School of Population and Global Health, The University of Melbourne, VIC, Australia, ${ }^{4}$ Department of Cancer Research, Peter MacCallum Cancer Centre, Melbourne, VIC Australia and ${ }^{5}$ Department of Medicine, The University of Melbourne, VIC, Australia

Background: Genomic risk assessments (GRAs) can stratify women's risk of developing breast cancer (BC), into clinically actionable risk categories. Women's attitudes on GRAs must be understood to inform implementation in a person-centered manner to promote population acceptance and participation. Aim: To explore women's attitudes of GRAs and determine what test-specific and test-delivery 
factors would influence their decision to participate. Methods: 31 women with no personal history of breast cancer, with and without a family history of $\mathrm{BC}$, participated in focus group discussions or personal interviews. Participants were explained the concept of BC-GRAs before discussing their perceptions of the test. Qualitative analysis was undertaken through inductive coding. Results: Participants responded positively. Identified themes include that acceptance hinges on the actionability of results, as women value the ability to inform decision-making and access risk-appropriate screening. Participants report they may be deterred by anxiety, stress, or the potential for genetic discrimination. Participants also highlighted the importance of the age GRAs are offered, how accessible they are, and how results are returned. Discussion: Women acknowledged effective implementation of GRAs relies on their ability to inform BC-risk mitigation. While women with a strong family history value the opportunity for GRAs to inform increased breast surveillance, women with low-risk results may feel discomfort accepting reduced breast surveillance as they find reassurance in regular screening. Meanwhile, women with no breast cancer family history may lack the stimulus to undertake genomic testing, which highlights the need for population education on the importance of engaging in GRAs and BC-risk mitigation.

\section{miRNA Regulation of Moesin in Lymphoma: A Deadly Relationship?}

E. K. Elliott, L. Hopkins, L. M. Haupt and L. R. Griffiths

Genomics Research Centre, Institute of Health and Biomedical Innovation, School of Biomedical Sciences, Queensland University of Technology, Brisbane, QLD, Australia

Non-Hodgkin's lymphoma (NHL) subtypes can be difficult to treat, even with an array of therapeutic agents on hand. Studies have highlighted Moesin (MSN) to be a potential marker of aggressive lymphoid malignancies such as Burkitt's lymphoma and DLBCL, and therefore a potential therapeutic target. To pave the way for improved targeted interventions, further understanding of the exact drivers behind $M S N$-initiated lymphomagenesis is required. To date, there is no clear literature on the relationship between miRNA:MSN regulation and NHL development and progression, therefore this project aims to undertake focused epigenetic and functional studies on MSN across various tissues to uncover its role in lymphomagenesis. Results to date have shown that specific miRNAs of interest regulate pathways, such as Janus kinases - signal transducer and activator of transcription proteins and signal transducer and activator of transcription proteins (STAT4), as well the regulation of critical cellular functions such as proliferation, formation of cytoskeleton, and cytokine signaling. MSN expression in NHL was upregulated in Burkitt's lymphoma and diffuse large Bcell lymphoma cell lines, suggesting MSN may prove to be a novel biomarker for risk and disease progression in specific NHL subtypes. Further investigations into miRNA:MSN targeting across NHL patient peripheral blood and tumor samples will enable a clearer understanding of $M S N$ 's involvement in critical pathways which are associated with NHL.

\section{'My New Normal': Experiences of Prophylactic Gastrectomy for Young Adults with a CDH1 Pathogenic Variant}

Rebecca Purvis ${ }^{1}$, Erin Tutty ${ }^{1}$, Cass Hoskins ${ }^{1}$, Mary Shanahan ${ }^{1}$, Alex Boussioutas ${ }^{1,2,3,4}$ and Laura Forrest ${ }^{1}$

${ }^{1}$ Parkville Familial Cancer Centre, Peter MacCallum Cancer Center and The Royal Melbourne Hospital, Melbourne, VIC, Australia, ${ }^{2}$ Department of Medicine, Royal Melbourne Hospital, University of Melbourne, Melbourne, VIC, Australia, ${ }^{3}$ Department of Gastroenterology, Royal Melbourne Hospital, Melbourne, VIC, Australia and ${ }^{4}$ Department of Surgical Oncology, Peter MacCallum Cancer Centre, University of Melbourne, Melbourne, VIC, Australia

Background: Unaffected individuals with a $\mathrm{CDH} 1$ pathogenic variant are recommended to have prophylactic total gastrectomy (PTG) from age 20 to 30 years to manage their significantly increased lifetime risk for diffuse gastric cancer. Despite the recommended timing of PTG, there is limited evidence of the impact of this major surgery on young adults; a distinct cohort with unique developmental considerations. Aim: This study aimed to explore the impact and experiences of PTG for young adults. Methods: Young adults aged 18-39 years with a $C D H 1$ pathogenic variant were invited from the Parkville Familial Cancer Centre to participate in a semistructured telephone interview. Interviews were audio-recorded, transcribed, and deidentified. Teambased thematic analysis was undertaken using an iterative and inductive process over several coding rounds. Results: Thirteen participants participated in interviews, nine of whom had proceeded with PTG. Participants reported a range of short- and long-term physical and psychosocial sequelae, experiencing more negative than positive impacts on their body image and recurrent hospitalizations. Most participants discussed extensive changes to their relationship with food and explored different adaptive strategies, including step-by-step and trial-and-error eating and altered social practices. Positive impacts of PTG included an increased sense of empowerment and control over cancer risk and stronger connections with social supports. Conclusion: This is the first study of its kind in Australia. These findings inform genetic counseling and gastroenterology practice by highlighting the need for holistic discussions of the possible impacts of PTG pre-surgery and the necessity of longer-term support as young adults navigate their new normal.

\section{Modeling Pathogenic Germline Variants to Understand Inherited Bone Marrow Failure and Predisposition to Myeloid Malignancy}

Parvathy Venugopal $^{1,2}$, Claire Homan ${ }^{1,2}$, Nur Hezrin Shahrin ${ }^{1}$, Peter Brautigan ${ }^{1,2}$, Rebecca Sawyer ${ }^{3}$, Paul Anderson ${ }^{3}$, Anna L. Brown ${ }^{1,2,3}$, Christopher N. Hahn ${ }^{1,2,3,6}$ and Hamish S. Scott ${ }^{1,2,3,5,6}$

${ }^{1}$ Department of Genetics and Molecular Pathology, SA Pathology, Adelaide, SA, Australia, ${ }^{2}$ Centre for Cancer Biology, SA Pathology \& University of South Australia, Adelaide, SA, Australia, ${ }^{3}$ UniSA Clinical \& Health Sciences, University of South Australia, Adelaide, SA, Australia, ${ }^{4}$ ACRF Cancer Genomics Facility, Centre for Cancer Biology, SA Pathology, Adelaide, SA, Australia, ${ }^{5}$ School of Biological Sciences, University of Adelaide, Adelaide, SA, Australia and ${ }^{6}$ Adelaide Medical School, University of Adelaide, Adelaide, SA, Australia

Background: Through the Australian Familial Hematological Cancer Study (AFHCS), we have identified causal germline variants in 
several inherited bone marrow failure disorders. We identified that heterozygous germline mutations in GATA2 underlie an array of complex hematopoietic and lymphatic diseases with a predisposition to leukemia. Aim and Methods: To define mechanisms by which pathogenic GATA2 variants cause disease, we have generated a mouse model representing the most common germline GATA2 (T354M) variant and characterized associated phenotypes. Results: Homozygous loss of GATA2 results in embryonic lethality between E10-10.5 in mice. Interestingly, in contrast to this, we have successfully generated Gata2 ${ }^{\mathrm{T} 354 \mathrm{M}}$ homozygous mice though they are born at a less-than-expected ratio. Consistent with previous in vitro findings, this indicates that the T354M variant is not a complete loss-offunction mutation. Peripheral blood and bone marrow analysis shows a marked reduction in lymphocytes and monocytes in Gata2 ${ }^{\mathrm{T} 354 \mathrm{M}}$ homozygous reflecting the immunodeficiency observed in patients. Furthermore, colony assays show a marked bias towards the granulocytic lineage and reduced macrophage differentiation. This model has also uncovered other potential underlying nonhematopoietic features such as osteopenia and urogenital abnormalities which are likely missed or underreported in patients. We are further investigating these mice, with particular emphasis on response to hematopoietic stressors and modeling autologous gene-edited stem cell transplantation, to better understand mechanisms of disease onset and prevent progression. Conclusion: Approaches to evaluate novel germline variants and uncover biological pathways affected in known germline pathogenic variants will provide insight facilitating advancements in diagnosis, personalized treatment, and possibly even prevention strategies.

\section{A Pilot Study Investigating the Health Economics of Achondroplasia in Australian Children}

Tessa Dentry, Supriya Raj and Ravi Savarirayan

Skeletal Therapies, Murdoch Children's Research Institute, Melbourne, VIC, Australia

Background: There are no approved therapies for achondroplasia that address the underlying pathophysiology. Vosoritide, a C-type natriuretic peptide analogue, has been developed by BioMarin Pharmaceutical and has demonstrated increased growth velocity in children with achondroplasia. If Therapeutic Goods Administration approval is granted for this potentially expensive medication, economic justification might assist in a Pharmaceutical Benefits Scheme listing/orphan drug designation, enabling all eligible Australian children to access this precision medication. Aim: To conduct a pilot study to better understand the economic impact of achondroplasia on the Australian healthcare system in children aged 0-5 years. Methods: Using standard of care information from The Royal Children's Hospital Melbourne and incidence/prevalence of complications, a cost analysis was performed to determine the approximate medical costs in this cohort. Results: A child with achondroplasia receiving standard care may cost the healthcare system between $\$ 27,787$ and $\$ 160,273$ by age 5 years, depending on the presence of associated medical complications such as hearing loss, otitis media, cervicomedullary compression, hydrocephalus and sleep apnea. Conclusions: These results demonstrate that the medical costs of children with achondroplasia are significant. Further investigation into other costs across the lifespan of this condition is required. The impacts this treatment may have on each individual, regardless of economic cost, must also be considered, as improvement in medical, functional, and psychosocial wellbeing have significant impacts on quality of life.

\section{Voretigene Neparvovec in Patients With RPE65 Mutation Associated Inherited Retinal Dystrophy: Baseline Characteristics From the Real-World, Prospective, Observational Perceive Study}

M. Dominik Fischer ${ }^{1,2}$, R Maier $^{3}$, Claudio Spera ${ }^{3}$, Bart P. Leroy ${ }^{4,5}$ and Christina Fasser ${ }^{6,7}$

${ }^{1}$ Centre for Ophthalmology, University of Tübingen, Tübingen, Germany, ${ }^{2}$ Oxford Eye Hospital, Oxford University Hospitals NHS Foundation Trust, Oxford, Oxfordshire, United Kingdom, ${ }^{3}$ Novartis Pharma AG, Basel, Switzerland, ${ }^{4}$ Department of Ophthalmology \& Center for Medical Genetics, Ghent University \& Ghent University Hospital, Ghent, Belgium, ${ }^{5}$ Children's Hospital of Philadelphia, Philadelphia, PA, United States, ${ }^{6}$ Retina International, Dublin, Ireland and ${ }^{7}$ Retina Suisse, Zurich, Switzerland

Background: Voretigene neparvovec (VN) is the first approved ocular gene therapy for treating patients with confirmed biallelic RPE65 mutation-associated inherited retinal dystrophy. PERCEIVE, a registry-based post-authorization safety study designed to assess longterm safety of VN in a real-world (RW) setting. Here, baseline characteristics of participating patients treated with $\mathrm{VN}$ are reported. Aim: The objective of the study is to characterize the long-term safety profile of $\mathrm{VN}$, to follow pregnancy outcomes, and to assess visual function over time. Methods: PERCEIVE is an ongoing, prospective, longitudinal, multicenter observational, safety study. All VN-treated patients are encouraged to participate in the study. After receiving $\mathrm{VN}$, patients are followed-up as per the routine medical care for 5 years. Results: At data cut-off, of the 15 patients enrolled, 10 patients (16 eyes) have received VN. At the time of enrollment, the mean (standard deviation [SD]) age was 27.6 (9.81) years with a range of 15-51 years (1 adolescent; 9 adults). For the treated eyes $(n=16)$, baseline mean $(\mathrm{SD})$ visual acuity was $0.96(0.37)$ logMAR ( $n=14$; range: $0.5-1.6 \operatorname{logMAR}$ ), and full-field stimulus threshold (white light) was $-4.32(9.40) \mathrm{dB}(n=15$; range: -34.9 to $3.2 \mathrm{~dB}$ ). Optical coherence tomography (OCT) revealed a mean foveal thickness of $150.5(41.07) \mu \mathrm{m}(n=15$; range: $76-216 \mu \mathrm{m})$. Central OCT showed that 11 eyes had an outer nuclear layer with a thickness range of $36-74 \mu \mathrm{m}(n=9)$, while it was absent in 4 eyes. The ellipsoid zone line was disrupted in nine eyes and was undetected in six. Conclusions: PERCEIVE provides valuable information on baseline characteristics of this vision-impaired population, and effect of VN therapy in a RW setting.

\section{Implementation of a Dedicated Ocular Genetics Clinic: Integrating Genetic Testing and Counseling into Ophthalmic Care}

Joshua Schultz ${ }^{1}$, Tiffany O'Brien ${ }^{1}$, Thomas L Edwards ${ }^{3,6}$, Jonathan B Ruddle ${ }^{3,4,5}$, Alex W Hewitt ${ }^{3,6}$, Thomas Campbell ${ }^{3}$, Lisa S Kearns ${ }^{3,6}$, Valentina Bartolo ${ }^{3}$, Lindsey Scotter ${ }^{3}$, Mark McCombe ${ }^{3}$, Paul James ${ }^{1}$, Ingrid Winship ${ }^{1,2}$ and Aamira Huq ${ }^{1,2}$

${ }^{1}$ Department of Genomic Medicine, Royal Melbourne Hospital, Melbourne, VIC, Australia, ${ }^{2}$ Department of Medicine, University of Melbourne, Melbourne, VIC, Australia, ${ }^{3}$ Royal Victorian Eye and Ear Hospital, Melbourne VIC, Australia, ${ }^{4}$ Department of Ophthalmology, University of Melbourne, Melbourne, VIC, Australia, ${ }^{5}$ Royal Children's Hospital, Melbourne, VIC, Australia and ${ }^{6}$ Centre for Eye Research Australia, Melbourne VIC, Australia

Historically, genetic testing for patients with inherited eye disease typically occurred through clinical genetics services based on nonspecialist hospitals or via research programs. With ophthalmology 
being at the forefront of gene therapy and treatment strategies, timely genetic testing is important in the delivery of expert care for these families. The Royal Melbourne Hospital (RMH) clinical genetics service and the Royal Victorian Eye and Ear Hospital (RVEEH) have initiated a combined multidisciplinary Ocular Genetics Clinic (OGC) based at the RVEEH to address this need. The service is comprised of ophthalmologists, orthoptists, clinical geneticist, and genetic counselors. A retrospective audit of the families seen in OGC covering the period between December 2018 and April 2021 was conducted by reviewing the medical records, with a focus on genetic testing and outcomes. In this period, 378 patients have attended OGC with a total of 259 genetic tests ordered, including diagnostic, predictive, confirmatory, and segregation testing. Of these, 116 diagnostic results have been received and disclosed to patients. The diagnostic rate during this time was $65 \%$, with pathogenic or likely pathogenic variants detected in 75 patients, confirming their clinical diagnosis. Eleven patients had variants of uncertain significance identified and are currently undergoing segregation. Of the remaining cases, a molecular diagnosis was not made in 30 patients. The implementation of the OGC is achieving its aims of providing expert care to families through increased access to genetic testing and identifying accurate diagnoses. This is critical in a field where treatment strategies are evolving rapidly.

\section{Active and Passive Implementation of Recommendations for Management of Rare Diseases: A Systematic Review}

Janet C Long $^{1}$, Stephanie Best ${ }^{1,2}$, Sarah Hatem ${ }^{1}$, Tahlia Theodorou ${ }^{1}$,

Jeffrey Braithwaite ${ }^{1}$ and John Christodoulou ${ }^{2,3}$

${ }^{1}$ Australian Institute of Health Innovation, Macquarie University, Sydney, NSW, Australia, ${ }^{2}$ Australian Genomics, Murdoch Children's Research Institute, Melbourne, VIC, Australia and ${ }^{3}$ Brain and Mitochondrial Research Group, Murdoch Children's Research Institute, and Department of Paediatrics, University of Melbourne, Melbourne, VIC, Australia

Background: Delivering evidence-based care to people with rare conditions is difficult. There is often scant high-quality evidence, health professionals may not have seen a presentation before, and conditions may be 'invisible' in the system. However, the recent rise of rare disease networks and international genomic alliances is contributing to a growing body of recommendations for rare diseases. Aim: To systematically review the peer-reviewed literature and to examine how recommendations were being implemented for the management of rare conditions. Methods: We searched Medline, EMBASE, Web of Science, and CINAHL using search strings for rare diseases AND practice guidelines, AND implementation/real-world use, with no date limits. Results: We found 495 articles with 103 undergoing full-text review. Only one article fully met our criteria of implementation of rare disease recommendations. This was a project across a region's emergency departments to optimize testing equipment, processes and staff knowledge for a time critical acute presentation of a rare disease. Most of the 103 papers described the development of consensus-based recommendations, reflecting the scarcity of evidence. The audience for the recommendations was often vaguely described and lacked specific information on who should do what. Many papers relied on passive dissemination by publication in a specialist journal, or flagged the inclusion of the guidelines in a rare disease repository. Discussion: Developers of consensus-based recommendations should be clear on the intended audience and have clear robust processes and an implementation plan/directions on how to operationalize the recommendations.

\section{Diagnosis of Low-Level Constitutional Mosaic Trisomy 12 by High-Resolution Microarray}

Krithika Murali ${ }^{1,2}$, David Francis ${ }^{1,2}$, Vida Petrovic ${ }^{1,2}$, Karen Dun ${ }^{3}$, Lyndon Gallacher ${ }^{1,2,6}$, Anand Vasudevan ${ }^{4}$, Colleen D'Arcy ${ }^{5}$, Rare Disease Flagship and Tiong Yang Tan ${ }^{1,2,6}$

${ }^{1}$ Victorian Clinical Genetics Services, Melbourne, VIC, Australia, ${ }^{2}$ Murdoch Children's Research Institute, Melbourne, VIC, Australia, ${ }^{3}$ Victorian Cancer Cytogenetics Service, Melbourne, VIC, Australia, ${ }^{4}$ Royal Women's Hospital, Melbourne, VIC, Australia, ${ }^{5}$ Royal Children's Hospital, Melbourne, Australia and ${ }^{6}$ Department of Paediatrics, University of Melbourne, Melbourne, VIC, Australia

Background/Aim: High-resolution microarray (HRM) provides more sensitive detection of constitutional chromosomal anomalies than single nucleotide polymorphism (SNP) array [Illumina Infinium GSA-24 v 2.0]. Methods: We first investigated an infant with atrial septal defect (ASD), right ventricular dysfunction, hypotonia, visual impairment, and dysmorphic features including blepharophimosis, posteriorly rotated low-set ears and anterior anus with SNP array and singleton exome sequencing (ES) performed on DNA extracted from saliva, which was uninformative. Subsequent HRM analysis [Illumina Infinium GDA-8 v1.0] was performed on DNA extracted from saliva. Fluorescence in-situ hybridization (FISH) on paraffin-embedded cardiac tissue was used as orthogonal confirmation of the HRM analysis. Results: HRM identified mosaic trisomy 12 in approximately $5 \%$ of cells in the saliva sample. The patient had undergone ASD repair with biopsy demonstrating interstitial fibrosis and endocardial fibroelastosis. FISH analysis of cardiac tissue confirmed mosaic trisomy 12 in $5 \%$ of cells (10 out of 200 cells analyzed). Discussion: HRM allowed for the detection of $5 \%$ trisomy 12 mosaicism in our patient, with the same finding validated using FISH on cardiac tissue. Mosaic trisomy 12 is a rare postnatal diagnosis. Affected individuals have a variable presentation most consistently characterized by facial dysmorphism, congenital heart disease, and hypotonia. The phenotype of our patient is consistent with that of other published cases of mosaic trisomy 12 in the literature. HRM should be considered for the detection of causative low-level mosaic chromosomal aneuploidies in those who remain undiagnosed after clinical exome and standard microarray.

\section{Delineation of the Clinical and Molecular Spectrum of QRICH1 Associated Neurodevelopmental Disorder}

Smitha Kumble ${ }^{1}$, Amanda M Levy $^{2}$, Jaya Punetha ${ }^{3,4}$, Hua Gao ${ }^{5}$, Nicholas Ah Mew ${ }^{6}$, Kwame Anyane-Yeboa ${ }^{7}$, Paul J Benke ${ }^{8}$, Sara M Berger ${ }^{7}$, Lise Bjerglund ${ }^{9}$, Belinda Campos-Xavier ${ }^{10,11}$, Michael Ciliberto ${ }^{12}$, Julie S Cohen ${ }^{13,14}$, Anne M Comi ${ }^{13,14}$, Cynthia Curry ${ }^{15}$, Lena Damaj ${ }^{16}$, Anne-Sophie Denommé-Pichon ${ }^{17,18}$, Lisa Emrick ${ }^{19,3}$, Laurence Faivre ${ }^{20,21}$, Alice Fiévet ${ }^{22,23}$, Richard S Finkel ${ }^{24,25}$, Sixto García-Miñaúr ${ }^{26,27}$, Amanda Gerard ${ }^{3,28}$, Paulino Gomez-Puertas ${ }^{29}$, Maria J Guillen Sacoto ${ }^{30}$, Trevor L Hoffman $^{31}$, Lillian Howard ${ }^{12}$, Alejandro D Iglesias ${ }^{32}$, Kosuke Izumi ${ }^{33}$, Austin Larson ${ }^{34}$, Anja Leiber ${ }^{35}$, Reymundo Lozano ${ }^{4}$, Iñigo Marcos-Alcalde ${ }^{29,36}$, Cassie S Mintz ${ }^{4}$, Sureni V Mullegama ${ }^{30}$, Rikke S Møller ${ }^{37,38}$, Sylvie Odent ${ }^{39}$, Henry Oppermann ${ }^{40}$, Elsebet Ostergaard ${ }^{41,42}$, Marta Pacio-Míguez ${ }^{26}$, Maria Palomares-Bralo ${ }^{26,27}$, Sumit Parikh ${ }^{43}$, Anna M Paulson ${ }^{12}$, Konrad Platzer ${ }^{40}$, Jennifer E Posey ${ }^{3}$, Lorraine Potocki ${ }^{3,28}$, Anya Revah-Politi ${ }^{44,45}$, Marlene Rio ${ }^{46}$, Alyssa L Ritter ${ }^{33}$, Scott Robinson $^{7}$, Jill A Rosenfeld ${ }^{3,47}$, Fernando Santos-Simarro ${ }^{26,27}$, Sérgio B Sousa ${ }^{10,48}$ Undiagnosed Diseases Network (UDN) ${ }^{49}$, Mathys Wéber ${ }^{20}$, Yili Xie ${ }^{30}$, Wendy $\mathrm{K}$ Chung $^{7}$, Natasha J Brown ${ }^{1,50, \#}$ and Zeynep Tümer ${ }^{2,42, \#}$

${ }^{1}$ Victorian Clinical Genetics Services, Murdoch Children's Research Institute, Melbourne, VIC, Australia, ${ }^{2}$ Kennedy Center, Department of Clinical Genetics, Copenhagen University Hospital, Rigshospitalet, Copenhagen, Denmark, ${ }^{3}$ Department of Molecular \& Human Genetics, Baylor College of Medicine, Houston, TX, USA, ${ }^{4}$ Department of Genetics and Genomic Sciences, Icahn School of Medicine at Mount Sinai, New York, NY, USA, ${ }^{5}$ Department of Review Analysis, GeneDx LLC, Maryland, USA, ${ }^{6}$ Rare Disease Institute, Children's National Hospital, Washington, DC, USA, ${ }^{7}$ Department of Pediatrics, 
Columbia University Irving Medical Center, New York, NY, USA, ${ }^{8}$ Division of Genetics, Joe DiMaggio Children's Hospital, Hollywood, FL, USA, ${ }^{9}$ Department of Pediatrics, University Hospital Hvidovre, Denmark, ${ }^{10}$ Medical Genetics Unit, Hospita Pediátrico, Centro Hospitalar e Universitário de Coimbra, ${ }^{11}$ Division of Genetic Medicine, Lausanne University Hospital and University of Lausanne (CHUV), Lausanne, Switzerland, ${ }^{12}$ Stead Family Department of Pediatrics, University of lowa, IA, USA, ${ }^{13}$ Department of Neurology and Developmental Medicine, Kennedy Krieger Institute, Baltimore, MD, USA, ${ }^{14}$ Department of Neurology, Johns Hopkins University School of Medicine, Baltimore, MD, USA, ${ }^{15}$ Genetic Medicine, Dept of Pediatrics, UCSF/Fresno, Fresno, CA, USA, ${ }^{16}$ Service de pédiatrie et de génétique clinique, CHU Rennes, France, ${ }^{17}$ INSERM UMR 1231 Equipe GAD, Université de Bourgogne, Dijon, France, ${ }^{18}$ Unité Fonctionnelle Innovation en Diagnostic génomique des maladies rares, FHUTRANSLAD, CHU Dijon Bourgogne, Dijon, France, ${ }^{19}$ Division of Neurology and Developmental Neuroscience, Department of Pediatrics, Baylor College of Medicine, Houston, TX, USA, ${ }^{20}$ Centre de Référence Anomalies du Développement et Syndromes Malformatifs, FHU TRANSLAD, Hôpital d'Enfants, CHU Dijon, Dijon, France, ${ }^{21}$ Inserm UMR1231 GAD, Génétique des Anomalies du Développement, Université de Bourgogne, Dijon, France, ${ }^{22}$ Laboratoire de biologie médicale multisites Seqoi-FMG2025, Paris, France, ${ }^{23}$ Service Génétique des Tumeurs, Gustave Roussy, Villejuif, France, ${ }^{24}$ Nemours Children's Hospital, Orlando, FL, USA, ${ }^{25}$ Center for Experimental

Neurotherapeutics, St. Jude Children's Research Hospital, Memphis, TN, USA, ${ }^{26}$ Institute of Medical and Molecular Genetics (INGEMM), La Paz University Hospital, Idipaz, Madrid, Spain, ${ }^{27}$ Centro de Investigación Biomédica en Red de Enfermedades Raras (CIBERER, U753), Instituto Carlos III, Madrid, Spain, ${ }^{28} \mathrm{Texas}$ Children's Hospital, Houston, TX, USA, ${ }^{29}$ Molecular Modeling Group, Severo Ochoa Molecular Biology Centre (CBMSO, CSIC-UAM), Madrid, Spain, ${ }^{30} \mathrm{Clinical}$ Genomics Program, GeneDx, Maryland, USA, ${ }^{31}$ Regional Department of Genetics, Southern California Kaiser Permanente Medical Group, Pasadena, CA, USA, ${ }^{32}$ Division of Clinical Genetics, Department of Pediatrics, Columbia University Irving Medical Center, New York, NY, USA, ${ }^{33}$ Divison of Human Genetics, Department of Pediatrics, Children's Hospital of Philadelphia, Philadelphia, PA, USA, ${ }^{34}$ Section of Genetics, Department of Pediatrics, University of Colorado School of Medicine, CO, USA, ${ }^{35}$ Department of neuropediatrics, Childrens Hospital of Eastern Switzerland, St. Gallen, Switzerland, ${ }^{36}$ Biosciences Research Institute, School of Experimental Sciences, Universidad Francisco de Vitoria, Pozuelo de Alarcón, Madrid, Spain, ${ }^{37}$ Department of Epilepsy Genetics and Personalized Treatment, The Danish Epilepsy Centre, Dianalund, Denmark, ${ }^{38}$ Department of Regional Health Research, University of Southern Denmark, Odense, Denmark, ${ }^{39} \mathrm{CHU}$ Rennes, Hôpital Sud, Service de Génétique Clinique, Univ Rennes, Centre de référence Anomalies du développement CLAD-Ouest, Rennes, France, ${ }^{40}$ Institute of Human Genetics, University of Leipzig Medical Center, Leipzig, Germany, ${ }^{41}$ Department of Clinical Genetics, Copenhagen University Hospital, Rigshospitalet, Copenhagen, Denmark, ${ }^{42}$ Department of Clinical Medicine, Faculty of Health and Medical Sciences, University of Copenhagen, Copenhagen, Denmark, ${ }^{43}$ Mitochondrial Medicine \& Neurogenetics, Cleveland Clinic, Cleveland, USA, ${ }^{44}$ Institute for Genomic Medicine, Columbia University Medical Center, New York, NY, USA, ${ }^{45}$ Precision Genomics Laboratory, Columbia University Irving Medical Center, New York, NY, USA, ${ }^{46}$ Service de Génétique, Hôpital Necker-nfants Malades, Assistance Publique-Hôpitaux de Paris (APHP), Paris, France, ${ }^{47}$ Baylor Genetics Laboratories, Houston, TX, USA, ${ }^{48}$ University Clinic of Genetics, Faculty of Medicine, University of Coimbra, Coimbra, Portugal, ${ }^{49}$ Undiagnosed Disease Network, UDN (member list in supplementary material) and ${ }^{50}$ Department of Paediatrics, University of Melbourne, Royal Children's Hospital, Melbourne, VIC, Australia

Background/Aim: De novo QRICH1 variants have been reported in 11 individuals with developmental delay, autism spectrum disorder, short stature, facial dysmorphism, and chondrodysplasia. We present 27 additional individuals to further delineate the phenotypic and genotypic spectrum of this emerging neurodevelopmental disorder. Methods: Phenotypic and molecular data were gathered through international collaboration and compared to those of the previously reported individuals. Results: Frequent phenotypic features included mild to moderate developmental delay/intellectual disability $(71 \%)$, nonspecific facial dysmorphism (92\%), and hypotonia (39\%). Additional findings were poor weight gain (29\%), short stature (29\%), autism spectrum disorder (29\%), seizures (24\%), and scoliosis (18\%). Minor structural brain abnormalities were reported in $52 \%$ of the individuals with brain imaging. Truncating or splice variants were found in 28 individuals and 10 had missense variants. Four variants were inherited from mildly affected parents. Conclusion: This study confirms that heterozygous QRICH1 variants cause a neurodevelopmental disorder and short stature and expands the phenotypic spectrum to include poor weight gain, scoliosis, hypotonia, minor structural brain anomalies, and seizures. Inherited variants from mildly affected parents are reported for the first time, suggesting variable expressivity. Conclusions regarding phenotypic differences between individuals with missense variants as compared to loss-of-function variants are limited by sample size.

\section{Brain Malformation and White Matter Abnormalities Associated With LSS-Related Neuroectodermal Syndrome}

Chloe A Stutterd ${ }^{1}$, Chirag Patel ${ }^{2}$, Ben Halliday ${ }^{3}$, Lisa Worgan ${ }^{4}$, Richard J Leventer ${ }^{1,5}$, Tung $\mathrm{Vu}^{6}$, Adriane Sinclair ${ }^{7}$, Adeline Vanderver ${ }^{8,9}$, Lisa Kratz ${ }^{10}$, Richard Kelley ${ }^{11}$, Mary Alice Abbott ${ }^{12}$, Stephen Robertson ${ }^{3}$ and Cas Simons ${ }^{1}$

${ }^{1}$ Murdoch Children's Research Institute and University of Melbourne, VIC, Australia, ${ }^{2}$ Genetic Health Queensland, Herston, Brisbane, QLD, Australia, ${ }^{3}$ Department of Women's and Children's Health, Dunedin School of Medicine, University of Otago, ${ }^{4}$ Department of Clinical Genetics, Liverpool Hospital, Sydney, Australia, ${ }^{5}$ Department of Neurology, Royal Children's Hospital, Melbourne, VIC, Australia, ${ }^{6}$ Paediatric Department, Gold Coast University Hospital, Southport, QLD, Australia, ${ }^{7}$ Department of Neurology, Queensland Children's Hospital, Brisbane, QLD, Australia, ${ }^{8}$ The Division of Neurology, Department of Pediatrics, The Children's Hospital of Philadelphia, Philadelphia, Pennsylvania, USA, ${ }^{9}$ The Department of Neurology, Perelman School of Medicine, University of Pennsylvania, Philadelphia, Pennsylvania, USA, ${ }^{10}$ Kennedy Krieger Institute, Baltimore, Maryland, USA, ${ }^{11}$ Department of Genetics and Genomics, Boston Children's Hospital, Boston, MA, USA and ${ }^{12}$ Medical Genetics, Department of Pediatrics, University of Massachusetts Medical School - Baystate, Springfield, MA, USA

Background: Disorders of cholesterol biosynthesis encompass a broad range of phenotypes with overlapping features, including intellectual disability, brain and limb malformations, and ectodermal defects. Bi-allelic mutations in LSS, encoding lanosterol synthase, recently were found to cause a spectrum of abnormalities beyond cataract, including hypotrichosis and a severe neuroectodermal syndrome (MIM\#618840) with some genotype-phenotype correlation. This phenotype has been recapitulated in a murine model with lanosterol synthase deficiency in affected tissue. Aim: To describe the neuroimaging abnormalities in patients with the LSS-associated alopecia and intellectual disability syndrome. Methods: Individuals with bi-allelic mutations in LSS and abnormal CNS neuroimaging were identified via international neurogenetic research collaborations. Previous reports of $L S S$-associated alopecia and intellectual disability syndrome were reviewed for neuroimaging abnormalities. Results: Four unrelated individuals were identified. CNS abnormalities in these individuals and previous reports include holoprosencephaly, cortical malformations, periventricular nodular heterotopia, cerebral atrophy, open opercula, cerebellar dysplasia, abnormal myelination, and corpus callosum dysgenesis. All four patients had congenital alopecia and epilepsy, and additional malformations included cleft palate, polydactyly, and genital hypoplasia. Sterol analysis identified an increased level of desmosterol in one individual, consistent with in vitro studies that show inhibition of desmosterol reductase by oxidosqualene as a feature of partial deficiency of LSS. Conclusion: 
LSS-associated neuroectodermal syndrome causes a spectrum of clinical features characterized by hypotrichosis and intellectual disability with or without epilepsy, brain malformation, and white matter abnormality. The cholesterol metabolism pathway is a potential target to modify the developmental and epileptic phenotype and warrants further investigation.

Incorporating the Patient Perspective: Systematic Review of Outcomes in Qualitative Studies Evaluating the Patient Experience of Reproductive Genetic Carrier Screening

Ebony Richardson, Alison McEwen, Toby Newton-John, Ashley Crook and Chris Jacobs Graduate School of Health, University of Technology Sydney, Sydney, NSW, Australia

Background: The Core Outcome Development for Carrier Screening (CODECS) study aims to develop a consensus-derived set of outcomes to measure in studies of reproductive genetic carrier screening (RGCS). This multistep process first involves reviewing published research literature to capture outcomes reported to date. In a previous systematic review of outcomes in quantitative studies offering RGCS, few studies included patient-reported outcomes. As a result, the patient perspective was lacking. Aim: To conduct a systematic review of qualitative studies reporting on the patient experience of RGCS, with the objective to identify outcomes of importance to patients. Methods: Medline, EMBASE, PsycINFO, and CINAHL were searched for published studies offering RGCS. Text excerpts capturing quotations and themes were extracted verbatim and a deductive analysis was conducted to code excerpts as research outcomes. Results: Sixteen studies from 2010 to 2020 were included. We identified 73 outcomes, belonging to 15 outcome domains. Most outcomes were consistent with those identified in the previous review of quantitative studies; however' reproductive confidence and empowerment were identified as an outcome unique to the qualitative literature. Conclusion: This is the first example of a qualitative approach for determining outcomes of importance to patients undergoing RGCS. The patient concept of personal utility, defined by reproductive confidence/empowerment, is absent from existing quantitative studies and suggests a novel dimension for future research to address. A core objective of the CODECS study is to involve patients in defining outcomes of importance for future research, which will enhance the relevance of research findings and maximize translation into patient-centred clinical practice.

\section{Intragenic de Novo Deletions In TAOK1 Resulting in Severe Neurodevelopmental Disorder}

Laura Raiti $^{1}$, David Francis ${ }^{1}$, Lyndon Gallacher ${ }^{1,3}$, Katrina M. Bell ${ }^{1}$, Simon Sadedin ${ }^{1}$, Lynn Pais ${ }^{2}$, David J. Amor ${ }^{1,3,4}$ and Zornitza Stark ${ }^{1,3}$

${ }^{1}$ Victorian Clinical Genetics Services, Murdoch Children's Research Institute, Melbourne, VIC, Australia, ${ }^{2}$ Center for Mendelian Genomics, Broad Institute of Harvard and MIT, Cambridge, MA, USA, ${ }^{3}$ The University of Melbourne,

Melbourne, VIC, Australia and ${ }^{4}$ The Royal Children's Hospital, Melbourne, VIC, Australia

Background: Single nucleotide variants in TAOK1 have recently been associated with a neurodevelopmental disorder characterized by intellectual disability, hypotonia, joint hypermobility, and early feeding difficulties. A range of molecular mechanisms has been proposed, including loss of function and dominant negative effects. Methods: Copy number variant analysis was performed on exome data from a child with profound neurodevelopmental disability. Additional cases were sought in an internal laboratory database. Findings were validated on a high-resolution SNP microarray platform. Results: A de novo intragenic deletion of exons 3-11 of TAOK1 was detected and confirmed on microarray. A second individual with a de novo deletion of exons 2-10 was identified. Both deletions result in the complete loss of the TAOK1 kinase domain. The affected individuals previously had uninformative clinical exome and microarray testing and have a strikingly similar phenotype comprising profound intellectual disability, microcephaly, and seizures, with a clinical diagnosis of cerebral palsy. Brain MRI demonstrated marked ventricular dilatation and decreased white matter in both children, with progressive cerebral and cerebellar atrophy on serial imaging in one. Discussion: Our findings suggest that intragenic deletions affecting the $\mathrm{N}$-terminal region kinase domain of TAOK1 result in a particularly severe phenotype. We highlight the importance of incorporating $\mathrm{CNV}$ analysis of exome data as part of the routine diagnostic practice. Small recurrent intragenic deletions in TAOK1 may be an under-recognized causative explanation for patients with similarly severe neurodevelopmental phenotypes.

\section{Childhood Prevalence of Achondroplasia in New South Wales (NSW) and the Australian Capital Territory (ACT), Australia}

Louise Tofts ${ }^{1,2}$, Jennifer Armstrong ${ }^{2,3}$ and Verity Pacey ${ }^{2,4}$

${ }^{1}$ Kids Rehab, The Children's Hospital at Westmead, NSW, Sydney, Australia, ${ }^{2}$ Department of Health Professions, Macquarie University, Sydney, NSW, Australia, ${ }^{3}$ Department of Orthopaedics, The Children's Hospital at Westmead, Sydney, NSW, Australia and 'Sydney Medical School, The Children's Hospital at Westmead Clinical School, University of Sydney, Sydney, NSW, Australia

The aim of this study was to estimate the childhood prevalence of achondroplasia, trends over time in birth prevalence, and age at diagnosis in Australia. Children born between 1990 and 2019 with a clinical and radiological and/or molecular diagnosis of achondroplasia were identified from a tertiary hospital servicing New South Wales (NSW) and the Australian Capital Territory (ACT) and compared with population data from the Australian Bureau of Statistics. Childhood prevalence of achondroplasia, based on children $\leq 19$ years of age and resident in NSW/ACT on June 30, $2019(n=109)$, was 5.2 per 100,000. A total of 127 individuals with achondroplasia were born in 1990-2019 in NSW/ACT. Birth prevalence rates increased across birth decades, from 3.3 per 100,000 live births in 1990-1999 to 5.3 per 100,000 in $2010-2019(p<.0001)$. Median age at diagnosis decreased to 17 days in 2010-2019 compared with 30 days in 1990-1999 ( $p=.035$ ), although the overall decreasing trend across consecutive decades did not reach statistical significance. This is the first study to show a rising birth prevalence rate for achondroplasia in Australia with a concurrent decreasing age at diagnosis, both of which were statistically significant after two decades.

\section{Mitochondrial Disease Diagnosis Via Next Generation Sequencing}

Justine Elliott ${ }^{1}$, Belinda Chong ${ }^{1}$, Daniel Flanagan ${ }^{1}$, Naomi Baker $^{1}$, Shannon Cowie ${ }^{1}$, David Thorburn ${ }^{1,4}$, John Christodoulou ${ }^{1,2,4}$ and Sebastian Lunke ${ }^{1,3}$

${ }^{1}$ Victorian Clinical Genetics Services, Murdoch Children's Research Institute, Melbourne, VIC, Australia, ${ }^{2}$ Department of Paediatrics, University of Melbourne, VIC, Australia, ${ }^{3}$ Department of Pathology, University of Melbourne, VIC, Australia and ${ }^{4}$ Murdoch Children's Research Institute, Melbourne, VIC, Australia

Background: Mitochondrial diseases represent a group of metabolic and neurological disorders characterized by defects in mitochondrial energy generation caused by mutations in mitochondrial DNA 
(single nucleotide variants (SNV) and larger deletions) and/or the nuclear genes encoding mitochondria. In 2019, VCGS introduced a new high coverage next generation sequencing (NGS) assay which simultaneously detects both (SNV) and large deletions in the mitochondrial genome, aiming to consolidate testing and increase diagnostic yield. Method: An audit of clinical data from the first 2 years of the NGS assay was undertaken to ascertain whether an increase in diagnostic yield could be achieved compared with a long-standing targeted assay containing a limited number of 22 common mitochondrial DNA mutations. Results: Data from 113 patients from across Australia was reviewed. Diagnostic results were issued for 34 patients (26 SNVs and 8 deletions). Potentially diagnostic variants of uncertain significance (Class $3 \mathrm{a}$ ) were identified in a further 10 patients and 69 negative results were reported. The overall diagnostic rate was $30 \%$. One patient was diagnosed with multiple deletions indicating a possible nuclear gene mutation and the need for subsequent exome or whole genome sequencing (WGS). Compared with the original targeted 22 variant panel, 18 additional diagnoses (8 deletions and 10 SNVs) were reported via the NGS assay, representing a doubling of diagnostic yield. Conclusion: Improved outcomes for patients can be achieved with increased diagnosis of mitochondrial disease by embracing rapidly advancing genomic technology, including whole mitochondrial genome analysis coupled with exome, or as part of WGS.

\section{Implementing a Patient-Centered Care Model to Diagnose Maturity Onset Diabetes of the Young (MODY)}

Rosalind Moxhamv ${ }^{1}$, Sophie Devery ${ }^{1}$, Renee Smythv, Alexander Viardot ${ }^{2,3}$, Jerry R Greenfield ${ }^{2,3}$ and Kathy $\mathrm{Wu}^{1,3,4,5}$

${ }^{1}$ St Vincent's Hospital Clinical Genomics, Sydney, NSW, Australia, ${ }^{2}$ Department of Endocrinology, St Vincent's Hospital, Sydney, NSW, Australia, ${ }^{3}$ St Vincent's Clinical School, UNSW, Sydney, NSW, Australia, ${ }^{4}$ School of Medicine, University of Notre Dame Australia, Sydney, NSW, Australia and ${ }^{5}$ Discipline of Genetic Medicine, University of Sydney, Sydney, NSW, Australia

Background: MODY is the most common monogenetic form of diabetes and often misdiagnosed as type 1 or type 2 diabetes. Currently, genetic testing is usually undertaken by genetics professionals following referrals to a genetics clinic. The implementation of a patientcentred model of care will allow nongenetics professionals to arrange MODY testing during routine clinical care. This care model is hypothesized to improve treatment experience and outcomes for patients. Aim: Implement a model of care for MODY genetic diagnosis; assess the comfort levels and support needs of Endocrinologists and psychosocial stress for patients; develop clinical guidelines and criteria for MODY testing. Methods: Eligible patients attending Diabetes Clinic are invited to participate by the treating endocrinologist. MODY testing is arranged following consent by endocrinologists at point-of-care. Results are returned by endocrinologists and used to guide patient management. Data are collected via patient demographic clinical data form at enrolment, modified Genetic Counseling Outcome Scale at pre- and post-testing, clinician experience questionnaire at the end of study. Analysis includes comparisons between pre-test and post-test scores and percentages used to summarize categorical data. Results: To date, 41 patients have been recruited by 15 endocrinologists. Of the patients who have received genetic testing results, a MODY diagnosis was achieved in $19 \%$ (4/21). Preliminary data showed all patients consider point-of-care MODY testing beneficial. There was an increase from $46 \%$ at pretest to $89 \%$ at posttest in patient's knowledge and confidence in sharing MODY information with their relatives. Conclusion: Mainstreaming MODY testing is feasible and beneficial for patients.

\section{A De Novo Missense Variant in MED13 in a Patient With Global Developmental Delay, Marked Facial Dysmorphism, Macroglossia, Short Stature, and Macrocephaly}

Alice P. Rogers ${ }^{1}$, Kathryn Friend ${ }^{1,2}$, Lesley Rawlings ${ }^{2}$ and Christopher P. Barnett ${ }^{1}$

${ }^{1}$ Paediatric and Reproductive Genetics Unit, Women's and Children's Hospital, Adelaide, SA, Australia and ${ }^{2}$ Genetic and Molecular Pathology, SA Pathology, Adelaide, SA, Australia.

Background: Variants in Mediator complex subunit 13 (MED13, OMIM 603808) have been reported in individuals with mild-moderate intellectual disability (ID) and/or developmental delay (DD). Aim: To expand the phenotypic spectrum of the MED13-associated syndrome by describing an additional individual found to have a de novo heterozygous missense variant in MED13 with more pronounced features than in the 20 individuals previously identified. Case Description: The proband was born at term following an unremarkable antenatal course. Early developmental milestones were significantly delayed. Sitting and walking did not occur until 3 and 4.5 years, respectively. The individual was nonverbal at 5 years of age, displayed autistic behaviors, and suffered from constipation. At 6 years of age, he was short (1st centile) and macrocephalic (99th centile). Marked dysmorphism was observed, including macroglossia, small midface, and down-slanting palpebral fissures. Results: A trio clinical exome analysis identified a likely pathogenic class IV de novo variant in the MED13 gene NM_005121.2:c.3449G>A (p.Gly1150Glu). MED13 comprises part of a four-subunit kinase module regulator (the 'Module') of Mediator. Mediator is a large multiprotein complex which promotes the transcription of protein-coding genes by RNA Polymerase II. The Module-Mediator complex, facilitated by MED13, is postulated to both promote and inhibit transcription. It follows that MED13 is a compelling candidate gene pertaining to neurodevelopmental disability. Conclusion: The neurodevelopmental profile of the proband is consistent with other described individuals, who were reported to have autistic features, speech delay, and constipation. However, this presentation was markedly more severe, expanding the phenotypic spectrum of MED13-associated syndrome.

\section{Qualitative Exploration of Patient and Caregiver Experiences of Visual Function Impairments and Impacts on Vision-Dependent Activities of Daily Living and Health- Related Quality of Life Associated With Retinitis Pigmentosa and Leber Congenital Amaurosis in Germany and France}

Isabelle Audo ${ }^{1}$, Nicola Williamson ${ }^{2}$, Helena Bradley ${ }^{2}$, Melissa Barclay ${ }^{2}$, Joel Sims ${ }^{2}$, Rob Arbuckle ${ }^{2}$, Francesco Patalano ${ }^{3}$, Claudio Spera ${ }^{3}$, Christel Naujoks ${ }^{3}$, Nuzhat Afroz ${ }^{4}$, M. Dominik Fischer ${ }^{5,7}$, Todd Durham ${ }^{8}$ and Judit Banhazi ${ }^{3}$

${ }^{1}$ Sorbonne Université, INSERM, CNRS, Institut de la Vision, CHNO des QuinzeVingts, DHU Sight Restore, INSERM-DGOS CIC1423, Paris, France, ${ }^{2}$ Adelphi Mill, Bollington, Cheshire, UK, ${ }^{3}$ Novartis Pharma AG, Basel, Switzerland, ${ }^{4}$ Novartis NBS, Hyderabad, India, ${ }^{5}$ Centre for Ophthalmology, University of Tübingen, Tübingen, Germany, ${ }^{6}$ Oxford Eye Hospital, Oxford University NHS Foundation Trust, Oxford, UK, ${ }^{7}$ Nuffield Laboratory of Ophthalmology, NDCN, University of Oxford, Oxford, UK, and ${ }^{8}$ Foundation Fighting Blindness, Columbia, MD, USA.

Background: Retinitis pigmentosa (RP) and Leber congenital amaurosis (LCA) are rare inherited retinal degenerative disorders (IRD). Visual impairments associated with these disorders have significant 
impacts on patients' vision-dependent activities of daily living (ADL) and broader health-related quality of life (HRQoL). There is a paucity of evidence exploring the patient experience of RP/LCA and how these impairments impact patients' lives across different genotypes. Aim: This study aimed to explore the experience of RP/LCA from patient and caregiver perspectives, across different genotypes and ages in Germany and France. Methods: Thirty semistructured qualitative concept elicitation interviews were conducted with 9 adults, 5 adolescents, 5 children, and 11 caregivers of children aged 3-11 years. The sample included different gene mutations linked to RP/ LCA: RPE65 $(n=11)$, RPGR $(n=5)$, CEP290 $(n=3), \operatorname{AIPL} 1(n=$ 2), LRAT, $P D E 6 B, R D H 12$, and $R P 2(n=1$ each). Thematic analysis of verbatim interview transcripts was performed. Results: Night blindness, peripheral vision, and contrast sensitivity limitations were the most frequently reported visual function symptoms. Severity of impairments depended on lighting conditions and familiarity of the environment. Proximal vision-dependent impacts included limitations to mobility and ADLs. Participants reported impacts on broader HRQoL domains like emotional wellbeing, social, and work/school functioning. Similar concepts were reported by caregivers. Conclusions: Visual function symptoms and impacts on vision-dependent ADLs and HRQoL were consistent across age groups and genotypes, suggesting the same measures may be appropriate for use in all RP/LCA types. Findings increased the understanding of the qualitative experience of RP/LCA and supported the development of fit-for-purpose patient- and observer-reported outcome measures for use in RP/LCA clinical trials.

\section{An Australian Monogenic Inflammatory Bowel Disease Cohort: Literature Review and Study Design}

Lavvina Thiyagarajan ${ }^{1}$, Manisha Chauhan ${ }^{1}$, Daniel Lemberg ${ }^{2}$, Nitin Gupta ${ }^{2}$, Paul Gray ${ }^{3}$ and David Mowat ${ }^{1}$

${ }^{1}$ Clinical Genetics, Sydney Children's Hospital Network, Sydney, NSW, Australia, ${ }^{2}$ Gastroenterology, Sydney Children's Hospital Network, Sydney, NSW, Australia and ${ }^{3}$ Immunology and Infectious Diseases, Sydney Children's Hospital Network, Sydney, NSW, Australia

Background: Inflammatory bowel disease (IBD) is a multifactorial disease resulting in chronic intestinal inflammation. The genetics of IBD is complex and mostly polygenic. Advances in NGS however, have revealed monogenic etiologies in a subset of patients. Monogenic IBD is rare, severe, and refractory to conventional therapies; however, descriptions of gene-specific treatment strategies are emerging. $\operatorname{Aim}(s)$ : (1) Understand the age distribution, clinical phenotype, and targeted therapies in monogenic IBD. (2) Curate a current IBD gene list. (3) Recruit an Australian cohort of potentially monogenic-IBD patients. (4) Apply genomic sequencing to study monogenic-IBD within this cohort. Methods: A literature review, addressing aim (1), was conducted and selection criteria identifying patients with likely monogenic-IBD were formulated. A clinically valid gene list was curated utilizing PanelApp and ClinGen guidelines. Patient recruitment has commenced through SCH. Results: The literature revealed more than 75 monogenic-IBD genes. Clinical features suggestive of monogenic-IBD include age of onset ( $<2$ years), family history, perianal disease, treatment failure, recurrent infections, and extraintestinal manifestations. Most monogenic-IBD is refractory to standard therapies. HSCT improved IBD in patients with some monogenic forms of IBD but had poor outcomes in others. The curated gene list has been implemented locally (SEALSPathology) and nationally (PanelAppAustralia - ongoing) and genomic testing has begun. Conclusion: The literature review identified criteria for pediatric
IBD patient prioritization for genomic sequencing and commenced the creation of a curated IBD gene list. We will continue recruitment through SCH and potentially other sites, to study the distribution of monogenic-IBD and the impact of genomics on treatment modalities.

\section{Qualitative Interviews With Patients and Caregivers Regarding Visual Function Impairments and Impacts on Vision-Dependent Activities of Daily Living and Health- Related Quality of Life in RPE65-Related Retinitis Pigmentosa and Leber Congenital Amaurosis}

Christine Kay ${ }^{1}$, Nicola Williamson ${ }^{2}$, Helena Bradley², Melissa Barclay ${ }^{2}$, Joel Sims², Rob Arbuckle ${ }^{2}$, Claudio Spera ${ }^{3}$, Christel Naujoks ${ }^{3}$, Nuzhat Afroz ${ }^{4}$, M. Dominik Fischer ${ }^{5,7}$, Isabelle Audo ${ }^{8}$, Todd Durham ${ }^{9}$, Francesco Patalano ${ }^{3}$ and Judit Banhazi ${ }^{3}$

${ }^{1}$ University of Florida, Gainesville, FL, USA, ${ }^{2}$ Adelphi Mill, Bollington, Cheshire, UK, ${ }^{3}$ Novartis Pharma AG, Basel, Switzerland, ${ }^{4}$ Novartis NBS, Hyderabad, India, ${ }^{5}$ Centre for Ophthalmology, University of Tübingen, Tübingen, Germany, ${ }^{6}$ Oxford Eye Hospital, Oxford University NHS Foundation Trust, Oxford, UK, ${ }^{7}$ Nuffield Laboratory of Ophthalmology, NDCN, University of Oxford, Oxford, UK, ${ }^{8}$ Sorbonne Université, INSERM, CNRS, Institut de la Vision, CHNO des Quinze-Vingts, DHU Sight Restore, Paris, France and ${ }^{9}$ Foundation Fighting Blindness, Columbia, MD, USA

Background: Inherited retinal degenerative disorders (IRD) such as Retinitis Pigmentosa (RP) and Leber Congenital Amaurosis (LCA) can be caused by mutations in the RPE65 gene. These mutations cause visual function impairments which have significant impacts on patients' vision-dependent activities of daily living (ADL) and broader health-related quality of life (HRQoL). There is limited evidence on the patient experience of RP and no known genotype-specific experiences. Aim: This study aimed to explore the experience of RPE65related RP/LCA from patient and caregiver perspectives. Methods: Semistructured qualitative concept elicitation interviews were conducted with 11 patients ( 7 adults, 1 adolescent, 3 children aged 611 years), and 5 caregivers of children aged 3-11 years. Patients had a clinical and genetic diagnosis of RPE65-related RP/LCA. Thematic analysis of verbatim interview transcripts was performed. Results: Night blindness, reduced peripheral vision, and problems with color vision were the most frequently reported visual function symptoms of RPE65-related RP/LCA. Proximal vision-dependent impacts included limitations to mobility and ADLs. Participants reported impacts on HRQoL domains including emotional wellbeing, social, financial, and work/school functioning. Severity of limitations varied based on lighting conditions and familiarity of the environment. Overall, similar concepts were reported across all participant groups. Conclusions: These findings provide in-depth insight into the visual function symptoms and impacts on vision-dependent ADLs and broader HRQoL associated with RPE65-related RP/LCA. Findings suggest that the visual function symptoms and impacts relevant to measure in clinical studies are consistent across RP/LCA genotypes. Interview findings contributed to the development of patient and observer-reported outcome measures specific to RP designed for use in clinical trials and to track disease severity in clinical practice.

\section{Cavitating Lung Lesions and Hemoptysis-A Rare Presenting Feature of Vascular Ehlers Danlos Syndrome}

Shannon LeBlanc ${ }^{1}$, Jeffrey Bowden ${ }^{2}$ and Christopher P. Barnett ${ }^{1}$

${ }^{1}$ Women's and Children's Hospital, Adelaide, SA, Australia and ${ }^{2}$ Southern Adelaide Local Health Network, Adelaide, SA, Australia

Background: The vascular type of Ehlers-Danlos syndrome (EDS IV), caused by pathogenic variants in the COL $3 a 2$ gene is the most 
severe type of Ehlers-Danlos syndrome, often presenting with catastrophic arterial or organ rupture. Pulmonary manifestations including recurrent pneumothorax are a recognized feature. Recurrent lifethreatening pulmonary hemorrhage is a rarely reported manifestation. Aim: To review the pulmonary manifestations of EDS IV by describing a family diagnosed with EDS IV following the death of a 17-year-old male who presented with recurrent hemoptysis. Case Description: The 17-year-old proband presented with an 18month history of intermittent hemoptysis, vomiting, abdominal pain, and weight loss. CTPA demonstrated bilateral cavitating lung lesions of unclear etiology. He died suddenly after collapsing at home. The family were referred for genetic investigation when the proband's brother presented with omental infarction and incidentally detected similar cavitating pulmonary lesions. Results: Clinical exome analysis identified a variant of unknown significance in the COL3a2 gene NM_000090.3:c.1754G $>$ T (p.585Val). Segregation testing demonstrated maternal inheritance. The proband's mother, who had a history of joint hypermobility, was subsequently found to have extensive arterial aneurysms consistent with EDS IV. The COL3a2variant segregated in a maternal uncle who also has features of a connective tissue disorder, and has been reclassified as likely pathogenic. Conclusion: Recurrent hemoptysis and cavitating lung lesions are a rarely reported manifestation of EDS IV, and should prompt consideration of an underlying connective tissue disorder. In this illustrative case, molecular diagnosis has enabled counseling and vascular surveillance for at-risk relatives.

\section{Outcomes of Genomic Sequencing for Families with Inherited Intellectual Disability - Where Next for Unresolved Families?}

Melanie Leffler ${ }^{1}$, Louise Christie ${ }^{1}$, Jackie Boyle ${ }^{1}$, Lucinda Murray ${ }^{1}$, Ellenore M. Marti ${ }^{1}$, E. Emma Palmer ${ }^{2,3}$, Tracy Dudding-Byth ${ }^{1,4}$, Anna Hackett ${ }^{1}$, Deborah Schofield ${ }^{5}$, Ying Zhu ${ }^{6}$, Michael Buckley ${ }^{6}$, Tony Roscioli ${ }^{3,6,7}$ and Michael Field ${ }^{1}$

${ }^{1}$ Genetics of Learning Disability (GOLD) Service, Department of Clinical Genetics, Sydney, NSW and Hunter Genetics, Sydney, NSW, Australia,, ${ }^{2}$ School of Women's and Children's Health, Faculty of Medicine, UNSW, Sydney, Australia, ${ }^{3}$ Sydney Childrens Hospital Network, Sydney, NSW, Australia, ${ }^{4}$ University of Newcastle, Newcastle NSW Australia, ${ }^{5}$ GenIMPACT: Centre for Economic Impacts of Genomic Medicine, Department of Economics, Faculty of Business and Economics, Macquarie University, Sydney, NSW, Australia, ${ }^{6}$ Randwick Genomics Laboratory - NSW Health Pathology, Prince of Wales Hospital, Sydney, NSW, Australia and ${ }^{7}$ Neuroscience Research Australia and Prince of Wales Clinical School, University of New South Wales, Sydney, NSW, Australia

Background: Inherited intellectual disability (ID) is highly heterogeneous, with over 700 monogenic causes. Genomic sequencing (exome/WES or genome/WGS) is the preferred platform for diagnostic approaches. Aim: To evaluate the diagnostic yield and clinical utility of genomic sequencing for families with two or more individuals with moderate-severe ID. Methods: Families known to the NSW GOLD service from 2008 to 2020 were assessed by the clinical team for suitability. Families were selected based on: (1) distinctive/severe phenotype (2) likelihood of resolving (3) clinical management (4) reproductive decision making. Whole genome sequencing was not available in Australia as an accredited diagnostic test at the time this project was conducted; therefore, sequencing was performed via Genome One or Australian Genome Research Facility with data analysis by the NSW Health Pathology Randwick Genomics laboratory. Results: Fifty-six families had genomic sequencing; 54 had WGS and two WES (one WES result pending). Twenty-one (38.2\%) had causative variants, two with novel genes identified. Three diagnoses impacted management. Several families required segregation and functional studies to validate variant pathogenicity. Families meeting all four criteria were more likely to have a diagnosis (44\% diagnostic yield) compared to three (33\%) or two (0\%) criteria. WGS identified at least one pathogenic variant that would not be identified via WES. Conclusion: Genomic sequencing has high utility for resolving families with complex inherited ID. Outcomes of this study have influenced clinical directions for unresolved families who will have their data reanalyzed in 2-3 years, and enrolment in research-based analyses as part of our GOLD Unresolved ID Program.

\section{Investigating Healthcare Professional and Parent Acceptability of a System Using Face-Matching Technology for Individuals With Developmental Disorders}

Stephanie Quattromani ${ }^{1}$, Jackie Boyle ${ }^{2}$, Tracy Dudding-Byth ${ }^{3}$ and Erin Turbitt ${ }^{1}$

${ }^{1}$ University of Technology Sydney, NSW, Australia, ${ }^{2}$ Hunter Genetics, Newcastle, NSW, Australia and ${ }^{3}$ The University of Newcastle, NSW, Australia

Background: The FaceMatch project aims to use face-matching technology to increase the diagnostic rate and novel gene discovery for children with syndromic intellectual disability. Aim: This study aimed to assess the acceptance of FaceMatch by parents and healthcare providers. We investigated attitudes and understanding of FaceMatch, as well as the barriers and enablers to participation. Methods: Semistructured interviews were conducted with healthcare professionals $(n=10)$ involved in diagnosing individuals with developmental disorders and parents $(n=7)$ of children with developmental disorders. Healthcare professionals were recruited through professional networks of the team and snowball sampling. Parents were recruited through support groups. Interview data were analyzed using thematic analysis. Results: Attitudes towards FaceMatch were mostly positive. Enablers to engagement for both cohorts included altruism, information-seeking behaviours, and the potential benefit of FaceMatch as a diagnostic aid. Healthcare professionals reported that uptake was dependent on the effectiveness of FaceMatch in providing diagnoses. Some uncertainty existed around the relevance of the technology and its effectiveness in providing a diagnosis. Time and resources were identified as the primary barrier. Parent-reported barriers included some concerns about privacy and the emotional toll of searching for a diagnosis. Conclusion: Understanding healthcare professional and parent acceptability informed improvements to the FaceMatch platform. This included the development of clear communication about the project objectives, process, and result delivery. Such improvements will improve engagement and recruitment of participants into FaceMatch and should facilitate improved diagnostic rates for individuals with developmental disorders.

\section{A Retrospective Cohort Study of Medical Complications in an Australian Population of Children with Achondroplasia}

Jennifer Armstrong ${ }^{1,2}$, Verity Pacey ${ }^{2,3}$ and Louise Tofts ${ }^{2,4}$

${ }^{1}$ Department of Orthopedics, The Children's Hospital at Westmead, Sydney, NSW, Australia, ${ }^{2}$ Department of Health Professions, Macquarie University, NSW, Australia, ${ }^{3}$ The Children's Hospital at Westmead Clinical School, University of Sydney, NSW, Australia, and and 'Kids Rehab, The Children's Hospital at Westmead, NSW, Australia, Sydney Medical School

Background and Objectives: Achondroplasia is the most common skeletal dysplasia resulting in short stature in humans. Medical 
complications of achondroplasia include craniofacial, ear, nose, and throat, orthopedic, respiratory complications, obesity, and death. This study aims to determine the rates of medical investigations, complications, and outcomes of interventions. Methods: Children and adolescents with Achondroplasia born between 2000 and 2019 with two or more episodes of care at our specialist clinic were included. Data were retrospectively collected from the clinical records on investigations, complications, interventions, and outcomes from 0 to 18 years of age. Standard descriptive statistics were used to analyze the frequency of these events and age of onset. Results: There were 108 participants, 58 males and 50 females. The mean length of follow-up was 9 years (SD 5.1 years) with median 29 (IQR32) outpatient and 2 (IQR5) inpatient attendances. Over 800 investigations were performed. There were $52(48 \%)$ participants with craniocervical stenosis, $15(13.9 \%)$ with hydrocephalus, 66 (61.1\%) with hearing impairment, $44(44.4 \%)$ with sleep disordered breathing, 46 (42.6\%) with lower limb malalignment, 24 (22.2\%) with thoracolumbar kyphosis, 10 (9.3\%) with symptomatic spinal stenosis, $12(11.1 \%)$ with obesity, and $16(14.8 \%)$ who had at least one admission for respiratory illness. 2 (1.9\%) children died during the study period. Conclusions: We report contemporary rates of medical complications in an Australian population of children with achondroplasia. This information will help guide clinicians with their expectant management of achondroplasia and provide context to families about the likelihood and timing of complications.

\section{CYTOGENETICS}

\section{A Spreadsheet to Determine Clonal Proportions in a Broad Range of Situations}

Ruth MacKinnon $1,2,3$

${ }^{1}$ St Vincent's Hospital, Melbourne, VIC, Australia, ${ }^{2}$ Melbourne Pathology, Melbourne, VIC, Australia and ${ }^{3}$ University of Melbourne, Melbourne, VIC, Australia

Background: Cancerous tissues usually include some normal cells. B allele frequencies (BAF) in SNP arrays can be used to estimate the proportions of different clones in mosaic cell populations. However, aside from simple ratios such as 2:1, estimating these proportions from the BAF is not straightforward. Aim: To produce a spreadsheet for calculating the proportions of two clones that are mosaic for copy number gain or loss. Methods: Our method is based on Illumina SNP arrays. B allele frequency is (number of B alleles)/ (total number of alleles). We reversed this formula to calculate the cell proportion when the copy number of the alleles in the two populations is known. We created a spreadsheet using this formula to determine clone proportions by inputting BAF and allele options. Results: The spreadsheet can be used to work out the proportions of two clones with known copy number differences. It also has wider applications: it can be used to work out the absolute proportion of A: $B$ alleles, which would, for example allow the degree of amplification to be determined $(n \mathrm{~A}: 1 \mathrm{~B})$. It can also be used where there is copy number neutral loss of heterozygosity $(\mathrm{CN}-\mathrm{LOH})$. It is therefore more versatile than tables that only cater for limited situations such as single copy loss or gain, or that are empirically derived. Conclusion: This spreadsheet is easy to use and can be used to determine the proportion of abnormal cells or the degree of amplification or $\mathrm{CN}-\mathrm{LOH}$.

\section{Two Unrelated Families with 5' Noncoding Duplications Upstream of MSX2 Refine the Critical Regulatory Region Likely Involved in Cranial Bone Development and a Cleidocranial Dysplasia-Like Phenotype}

Mark Cleghorn $^{1, \star}$, Prabhakara Krishnamurthy ${ }^{1, \star}$, Tegan French $^{1}$, David Francis ${ }^{2}$ and Tiong Yang $\operatorname{Tan}^{3}$

${ }^{1}$ Victorian Clinical Genetics Services, Melbourne, VIC, Australia, ${ }^{2}$ Murdoch Children's Research Institute, Melbourne, VIC, Australia and ${ }^{3}$ Department of Paediatrics, University of Melbourne, Melbourne, VIC, Australia

Background: Intragenic MSX2 variants result in distinct phenotypes including craniosynostosis and parietal foramina with or without cleidocranial dysplasia. We present two unrelated families with delayed closure of a wide anterior fontanelle and cranial sutures with inherited duplications upstream of MSX2. Methods: Genomic DNA was extracted from peripheral blood/saliva samples of the probands and their parents. Microarrays were performed using Illumina Infinium GSA-24 v2.0 (Family 1) and CoreExome-24 v1.1 (Family 2). Results: Patient 1 presented at 2 months with wide anterior fontanelle, clinodactyly, and normal clavicles. Microarray identified a 1.5 Mb duplication of chromosome 5q35.1q35.2 (hg19 chr5:172,412,553-173,888,281), 259kB upstream of MSX2. His mother had $40 \%$ mosaicism for the same duplication, but no skeletal or cranial abnormalities. Patient 2 presented at two months with wide anterior fontanelle and cranial sutures, but normal clavicles. Microarray identified a $600 \mathrm{~kb}$ duplication of chromosome $5 \mathrm{q} 35.2$ (chr5:173,416,324-174,025,065) $122 \mathrm{~kb}$ upstream of MSX2. Parental segregation identified maternal inheritance. Her mother in childhood had similar features with closure of her anterior fontanelle at 2.5 years. She is of normal adult height and cognition without dental anomalies. Conclusions: These duplications overlap with those previously reported in a $219 \mathrm{~kb}$ noncoding region upstream of $M S X 2$, which contains several evolutionarily conserved noncoding elements that may act as specific enhancers of MSX2. These duplications are likely to alter the topological landscape around MSX2. Our observations strengthen the evidence for pathogenicity of copy number variations in this region and refine the critical region that may result in MSX2 dysregulation and a cranial phenotype.

\section{Preliminary Study on the Cytogenomics of Uveal Melanoma}

Rachel Fraser, Mario Nicola, Jeff Suttle, Chadd Gabell, Tony Hillier, Simone Bawden, Mahmoud Khazab, Zac Boaden, Lisa Huynh, Chelsea Falco, Sarah Moore, Amardeep Gilhotra, David Sia and Chun Chun Young

SA Pathology, Adelaide, SA, Australia

Ocular melanoma, although rare, is the most common primary cancer of the eye in adults. $83 \%$ of tumors arise in the uvea (UM). First-line treatment options are resection, radiation therapy, and enucleation which provide good local disease control, but long-term survival is reduced by metastatic disease. UM exhibits recurring chromosomal abnormalities and gene mutations, which strongly correlate with tumor evolution and metastatic risk and because of this accurate cytogenomic profiling of UM tumor samples has become increasingly important. By identifying adverse genetic markers early in the disease, the risk of metastasis will be reduced and long-term survival rates will increase. Paraffin FISH has been used to identify the clinically relevant copy number variants $(\mathrm{CNV})$ such as the high risk markers: loss of BAP1 from chromosome 3, loss of $1 \mathrm{p}$ 
and gain of the long arm of chromosome 8 ; and the good risk marker: gain of $6 \mathrm{p}$. We have introduced SNP array, which offers a genomewide screen for detection of all UM CNV. SNP array has the added advantage of demonstrating copy neutral loss of heterozygosity, which may occur in patients who have BAP1 mutation. Although genetic studies are easily performed on advanced tumors and at enucleation, one of the problems of early diagnosis of ocular melanoma is the small tissue size at vitrector or fine needle sampling. We report our early laboratory experience with extremely small UM biopsies and the use of different procedures to provide sufficient DNA for SNP array.

\section{Genomewide NIPT Complements SNP Microarray to Confirm Trisomy Rescue Events}

Kasey Peachey ${ }^{1}$, Wendy Waters ${ }^{1}$, Tristan SE Hardy ${ }^{1,3}$, Sarah Higgins ${ }^{1}$

Shanna Suwalski ${ }^{1}$, Kathie Friend ${ }^{1}$, Luis Perez-Jurado ${ }^{2}$ and Sui $\mathrm{Yu}^{1}$

${ }^{1}$ Genetics and Molecular Pathology, Adelaide, SA, Australia, ${ }^{2}$ Paediatric and Reproductive Genetics Unit, Women's and Children's Hospital, Adelaide, SA, Australia and ${ }^{3}$ Repromed, Dulwich, SA, Australia

SNP microarray analysis is commonly requested at prenatal diagnosis for high-risk pregnancies. SNP microarray can detect genomic gain and loss, lower levels of mosaicism than conventional karyotyping, and copy neutral regions of homozygosity ( $\mathrm{ROH})$. $\mathrm{ROH}$ can indicate close common parental ancestry and uniparental disomy (UPD). Recently, noninvasive prenatal testing (NIPT) has been widely accepted as a screening tool for common aneuploidies. NIPT uses fetal (placental) DNA circulating in the maternal circulation. Although NIPT is sensitive and specific, false-positive results occur, with confined placental mosaicism (CPM) involving aneuploidy being a common cause. CPM is commonly associated with intrauterine growth restriction, pregnancy loss, or perinatal death in some cases. CPM can also be a marker for UPD. Four cases will be described where NIPT has indicated a trisomy and subsequent prenatal diagnosis with SNP microarray has detected $\mathrm{ROH}$ on the chromosome of interest; a case each of trisomy 16, trisomy 2, trisomy 21, and trisomy 13. Further investigation has confirmed UPD in two cases and has inferred CPM in the other cases. While the cases presented do not involve a chromosome with known imprinted genes, there is a risk of unmasking an autosomal recessive disease and an undetected residual trisomy cell line cannot be excluded.

\section{EDUCATION, ETHICS AND SOCIAL ISSUES}

\section{An Educational Framework for Continued Professional Development of Genetic Counselors Through Introduction of Advanced Counseling Workshops}

Lisa Gordon ${ }^{1}$, Linda Cicciarelli ${ }^{1,2}$ and Maira Kentwell ${ }^{1,3,4}$

${ }^{1}$ Parkville Familial Cancer Centre and Genomic Medicine, Melbourne, VIC, Australia, ${ }^{2}$ Department of Paediatrics, University of Melbourne, Parkville, VIC, Australia, ${ }^{3}$ Department of Oncology and Dysplasia, The Royal Women's Hospital, Melbourne, VIC, Australia and ${ }^{4}$ Department of Medicine, University of Melbourne, Melbourne, VIC, Australia

Continued professional development (CPD) is a routine component of genetic counseling practice to ensure practising genetic counselors maintain their knowledge and skills. To support our genetic counselors in continuing to build their counseling skills, we have developed an educational framework centered around Kolb's theory of experiential learning, which focuses on advanced counseling skills. Through a series of facilitated workshops, counselors engaged in a cycle of activity, reflection, learning, and application aimed to provide further skills training, an opportunity to increase awareness of counseling theory and applying it to genetic counseling practice. This learning opportunity enabled a large genetic counseling team with a breadth of experience to participate. We describe the process of using an external facilitator (a specialist in counseling) to teach advanced counseling skills and deepen how we integrate these skills into genetic counseling practice. A genetic counselor was assigned to this brief working closely with the facilitator to obtain feedback from participants after each workshop. This was taken on in planning future workshops. At the end of the year, a semistructured questionnaire was administered to participants evaluating whether the workshops had met their CPD learning needs. Participants were asked to consider theoretical content, method of delivery, and application to clinical practice. Suggestions were invited to inform future workshop planning. The outcome of the first year in implementing advanced counseling workshops was valued by the team, as it increased awareness of how we can integrate counseling skills and theory more effectively into our practice. We conclude this experiential learning education framework is beneficial to meet CPD learning needs.

\section{Person-Centered Genomics: Experiences of Queensland Genomics Community Advisory Group}

Miranda E. Vidgen ${ }^{1}$, Katrina Cutler ${ }^{2,3}$, Jessica Bean ${ }^{4}$, David Bunker ${ }^{2,3,4}$, Lindsay F. Fowles ${ }^{4,5}$, Louise Healy ${ }^{4}$, Gary Hondow ${ }^{4}$, Satrio Nindyo Istiko ${ }^{4}$, Aideen M. McInerneyLeo $^{4,6}$, Gregory Pratt ${ }^{1,4}$, Deborah Robins ${ }^{4}$, Nicola Waddell ${ }^{1,4}$ and Erin Evans ${ }^{4,7}$

${ }^{1}$ QIMR Berghofer Medical Research Institute, Brisbane, QLD, Australia, ${ }^{2}$ Queensland Genomics, Brisbane, Qld, Australia, ${ }^{3}$ Brisbane Diamantina Health Partners, Brisbane, QLD, Australia, ${ }^{4}$ Queensland Genomics Community Advisory Group, Brisbane, QLD, Australia, ${ }^{5}$ Genetic Health Queensland, Royal Brisbane and Women's Hospital, Brisbane, QLD, Australia, ${ }^{6}$ Dermatology Research Centre, The University of Queensland, Brisbane, QLD, Australia and ${ }^{7}$ Health Consumers Queensland, Brisbane, Qld, Australia

Background: Consumer and community engagement has become a priority in health and medical research. To address this priority, Queensland Genomics established a Community Advisory Group (CAG) with dual role of advisory committee and leaders in community-centred activities. Aim: To summarize the learnings from a CAG in addressing the challenges regarding community engagement in genomic research and service delivery. Methods: The CAG's structure and function were captured by analyzing documentation from Queensland Genomics and the CAG. The experiences of CAG members $(n=11)$ were provided through interviews, group discussions, and written submissions. Results: In its advisory capacity, CAG provided advice to some Queensland Genomics projects, contributing to governance review, participating in the project selection process, and designed project reporting on consumer and community engagement. The CAG was provided resources to develop and lead community-focused projects. The CAG Projects address four areas; informing the community, workforce development, supporting genomic consumers, and research from the consumer perspective. The main barrier experienced by the CAG centred on ability or willingness to incorporate CCE in health service planning and projects. This barrier predominately affected the advisory capacity of the CAG. Key enablers for the dual roles of the CAG included; diversity of CAG members' skills and experience, relationships (within CAG, between CAG and Program and CAG member networks), resourcing (financial and human), and the CAG's ability to self-determine their direction. Conclusion: When appropriately structured, resourced, and supported, CAGs can effectively identify and lead consumer-focused projects with health implementation programs for genomics. 


\section{Health Interpreter Training in Genetic Terminology: An Update on Delivery and Evaluation}

Miranda E. Vidgen ${ }^{1}$, Lindsay F. Fowles ${ }^{2,3}$, Satrio Nindyo Istiko ${ }^{3}$, Erin Evans ${ }^{3,4}$, Katrina Cutler ${ }^{5,6}$, Kate Sullivan ${ }^{5}$, Jessica Bean ${ }^{3}$, Louise Healy ${ }^{3}$, Gary Hondow ${ }^{3}$, Aideen M. Mclnerney-Leo ${ }^{3,7}$, Gregory Pratt ${ }^{1,3}$, Deborah Robins ${ }^{3}$, Stephanie Best ${ }^{8,9}$, Keri Finlay ${ }^{8,10}$, Priya Ramarao-Milne ${ }^{1}$ and Nic Waddell ${ }^{1,3}$

${ }^{1}$ QIMR Berghofer Medical Research Institute, Brisbane, QLD, Australia, ${ }^{2}$ Genetic Health Queensland, Royal Brisbane and Women's Hospital, Brisbane, QLD, Australia, ${ }^{3}$ Queensland Genomics Community Advisory Group, The University of Queensland, Brisbane, QLD, Australia, ${ }^{4}$ Health Consumers Queensland, Brisbane, QLD, Australia, ${ }^{5}$ Queensland Genomics, The University of Queensland, Brisbane, QLD, Australia, ${ }^{6}$ Brisbane Diamantina Health Partners, Brisbane, QLD, Australia, ${ }^{7}$ Dermatology Research Centre, The University of Queensland, Brisbane, QLD, Australia, ${ }^{8}$ Australian Genomics, Murdoch Children's Research Institute, Melbourne, VIC, Australia, ${ }^{9}$ Macquarie University, Sydney, NSW, Australia and ${ }^{10}$ Genetic Support Network of Victoria, VIC, Australia

Background: Health Interpreters provide a vital service within the health system for patients with limited English. Language skills in specialist areas tend to come from work-based practise or post-certification professional development. Aim: To delivery and evaluation of a training session for Health Interpreters on genetic concepts. Methods: In July/August 2020, three 2-hour online training sessions on genetic concepts were held in English with a cohort of Health Interpreters. The training program included presentations on basic and applied genetics, two interactive activities. A recording of training has been edited to a 1 -h seminar that is available online. Evaluation of the 2 -h training session was undertaken with pre- and post-event surveys and a 6month follow-up survey. These surveys explored changes in participant knowledge, self-efficacy, attitudes and behaviour, and session experience. Results: 118 interpreters participated in the training sessions, and there have been a further 116 views of the recorded seminar. Respondent knowledge improved and gains were maintained at 6 months $(p<.01)$. There were no changes in self-efficacy, attitudes, and self-reported practice. Self-reported practice behaviour varied considerably between participants, but was not changed by the training. Most respondents agreed that training was useful (93\%) and relevant $(79 \%)$ to their work. Conclusion: Health Interpreters found the training acceptable and demonstrated sustained improvement in knowledge of genetic concepts. Increased delivery of this training and associated research is needed to assess the impact of this educational intervention on patients.

\section{Collaborative Development of Educational Resources to Support Medicare-Rebated Genomic Testing for Childhood Syndromes/Intellectual Disability (ID)}

Natalie Grainger ${ }^{1}$, Mike Field ${ }^{2}$, Ellenore Martin ${ }^{2}$, Lucinda Murray ${ }^{2}$, Hossai Gul ${ }^{3,4}$, Anna Hackett ${ }^{2}$, Marina Maxhuni ${ }^{1}$ and Gayathri Parasivam ${ }^{1}$

${ }^{1}$ Centre for Genetics Education NSW Health, Sydney, NSW, Australia, ${ }^{2}$ Genetics of Learning Disability (GoLD) Service, Sydney, Australia, ${ }^{3}$ Australian Institute of Health Innovation, Macquarie University, Sydney, NSW, Australia and ${ }^{4} \mathrm{NSW}$ Agency for Clinical Innovation, Sydney, NSW, Australia

Background: Medicare-rebated whole exome or whole genome sequencing can now be ordered for eligible patients by pediatricians in consultation with clinical geneticists. Surveys $(n=102)$ and interviews $(n=19)$ of pediatricians identified barriers and facilitators to ordering testing. Resources to assist with delivering results to families were reported as a priority need for pediatricians. Aim: Develop resources to enhance pediatricians' knowledge and confidence. Methods: Over a 6-month period, we used codesign and stakeholder feedback to determine the best approach to developing resources for rare genetic conditions. Results: A priority list of causative genes for ID was created based on disease frequency per the Decipher database and an audit of online resources. Feedback from pediatricians indicated a preference for resources that would help communicate genomic test results to families and assist with clinical management. Resources were designed to incorporate point-of-care guidance for pediatricians and disease-specific information for families. Iterative review with pediatricians (primary target audience), patient advocates (representing the secondary audience), and rare disease experts including genetic counselors and clinical geneticists yielded a fact sheet template written to a reading age of $\sim 14-15$ years. Clinical content experts are using this template to develop $\sim 20$ fact sheets with the assistance of a multidisciplinary Review Committee and team members with experience in medical education. Early feedback indicates that this novel approach meets the needs of the target audience. Conclusion: Utilising a multidisciplinary team incorporating medical education expertise can generate purpose-built resources quickly and effectively. This approach can be applied to future resource development for genetic disorders.

\section{Referral Pathways: Integrated Genetic Healthcare for Aboriginal and Torres Strait Islander Queenslanders}

Victoria Donoghue ${ }^{1}$, Greg Pratt ${ }^{2}$, Kelly Dingli ${ }^{3}$, Kate Houghton ${ }^{4}$, Angela Young ${ }^{5}$ and Julie McGaughran ${ }^{6}$

${ }^{1}$ QIMR Berghofer/QAIHC, Brisbane, QLD, Australia, ${ }^{2}$ QIMR Berghofer, Brisbane, QLD, Australia, ${ }^{3} \mathrm{QAIHC}$, Brisbane, QLD, Australia, ${ }^{4}$ Genetic Health Queensland, Brisbane, QLD, Australia, ${ }^{5} \mathrm{QAIHC}$, Brisbane, QLD, Australia and ${ }^{6} \mathrm{Genetic}$ Health Queensland/School of Medicine, University of Queensland, Brisbane, QLD, Australia

Background: Advances in genomic technologies promise to revolutionize healthcare. It is essential we identify barriers to Aboriginal and Torres Strait Islander peoples' engaging with genetic health services. Aim: To support Aboriginal and Torres Strait Islander Community Controlled Health Organizations (ATSICCHOs) of Queensland identify preferences and recommendations for a pathway to sensitive and integrated genetic healthcare. Methods: Workshops facilitated across Queensland sought advice from service stakeholders and the community about barriers and enablers to access, quality experience of genetic testing and counseling for Aboriginal and Torres Strait Islander Queenslanders. Thematic analysis identified key themes and informed the development of documentation outlining recommendations to realize a goal of integrated healthcare for ATSICCHO patients accessing Genetic Health Queensland (GHQ). Results: We describe topics and recommendations identified through workshops for core themes: (1) workforce, (2) coordinated care, and (3) public awareness. The penultimate version of the integrated healthcare model will be presented, emphasising the role of co-design and partnership between the public and community-controlled health service sector; describing recommendations for action that support Aboriginal and Torres Strait Islander access to quality genetic healthcare. Participants reviewed and confirmed the Integrated Genetic HealthCare (IGHC) model as inclusive and representative of their advice. Conclusion: ATSICCHOs champion patient access to quality healthcare for Aboriginal and Torres Strait Islander peoples. Partnership between ATSICCHOs, the public hospital, and health services that is GHQ has enabled development of the pathway and should lead to improved access to sensitive, appropriate, and quality health genetic services for Aboriginal and Torres Strait Islander Queenslanders. 


\section{Developing Capacity in Genomic Medicine Through Implementation Focused Training}

Rehan Villani ${ }^{1}$, Lindsay Fowles ${ }^{1,2}$, Elizabeth Peach ${ }^{1}$, Jess Schluter ${ }^{1}$, Sarah Steinke ${ }^{1}$, Kaye Hewson ${ }^{1}$ and Chirag Patel ${ }^{1,2}$

${ }^{1}$ The Genomic Institute, Metro North Health, Brisbane, QLD, Australia and ${ }^{2}$ Genetic Health Queensland, Metro North Health, Brisbane, QLD, Australia

Background: Rapid advances in genomic technologies are providing opportunities for significant improvement in clinical care. One major challenge to implementing genomics into widespread clinical care is insufficient capacity in our trained healthcare workforce to facilitate the rapidly increasing availability of genomic technologies. It is imperative that we put frameworks in place to enable genomics for best practice and efficient patient care. Aim: The Genomic Institute is a Metro North Health-led initiative that aims to facilitate the mainstreaming of genomic care into the Queensland healthcare system by developing strong and implementable training in genomics practice. Methods: There have been considerable efforts, Australia and worldwide, to assess priority areas and methods for genomics training. The Genomic Institute is using this evidence base to implement a training program in genomics, to enable mainstream use of genomics. Results: The Genomic Institute educational framework includes genomics foundations training complemented by speciality specific training, initially in renal, maternal fetal medicine, primary care, nursing, and midwifery but also incorporating local needs assessment for future development. The program has successfully integrated partner initiatives and has early indicators supporting high impact. Evaluation is integrated into the programs, to inform future scope and assess the impact and outcomes of the genomics education. Conclusion: To fully capitalize on developing genomics technologies, it is essential to mainstream genomics implementation. The Genomic Institute educational program is being developed to facilitate efficient and evidence-based training to ultimately improve the delivery of care and patient outcomes.

\section{An Ethical Decision-Making Framework for Genomic Testing in Pediatric Kidney Disease}

Inez Beadell ${ }^{1}$ and Catherine Quinlan ${ }^{1,2,3}$

${ }^{1}$ Department of Paediatrics, University of Melbourne, Melbourne, VIC, Australia, ${ }^{2}$ Department of Paediatric Nephrology, Royal Children's Hospital, Melbourne, VIC, Australia and ${ }^{3}$ Department of Kidney Regeneration, Murdoch Children's Research Institute, Melbourne, VIC, Australia

Background: Technological advances and increased access have led to genomics expanding beyond the genetics speciality. As a result, nongenetics specialists, including nephrologists, can now order genomic testing for their patients. Consistent decision-making around patient selection is required to ensure equitable access while maximising the utility of genomic testing, from a patient and resource perspective. However, there are currently no frameworks to guide decision-making for genomic testing in pediatric nephrology. Aim: To develop an ethical decision-making framework to guide patient selection for genomic testing in pediatric kidney disease. Methods: A three-staged approach was used: (1) review of the literature on decision-making for genomic testing in nephrology and other disciplines; (2) observations of approaches to genomic testing in the general nephrology clinic and the renal genetics clinic at the Royal Children's Hospital; (3) review and revision of the framework with key stakeholders, including clinical geneticists, genetic counselors, pediatric nephrologists, clinical ethicists, and families from the renal genetics service. The initial framework was modified until consensus from all key stakeholders was reached. Results: A decision-making framework was created. This framework outlined the key decision-making categories and sub-categories for patient selection, with corresponding questions to aid usage. A number of case studies were also developed to demonstrate the framework's application. Conclusion: This framework will guide decisions around patient selection for genomic testing in nephrology at the Royal Children's Hospital as well as other institutions and disciplines. In doing so, it will facilitate consistent approaches to genomic testing, to maximize equity and utility.

\section{Resources for Navigating Genetic Health Services in Queensland: The Patient Communication Toolkit}

Miranda E. Vidgen ${ }^{1}$, Katrina Cutler ${ }^{2,3}$, Kate Sullivan ${ }^{2}$, Gary Hondow ${ }^{4}$, Jessica Bean ${ }^{4}$, David Bunker ${ }^{2,3,4}$, Lindsay F. Fowles ${ }^{4,5}$, Louise Healy ${ }^{4}$, Satrio Nindyo Istiko ${ }^{4}$, Aideen M. McInerney-Leo ${ }^{4,6}$, Gregory Pratt ${ }^{1,4}$, Deborah Robins ${ }^{4}$, Nicola Waddell ${ }^{1,4}$ and Erin Evans ${ }^{4,7}$

${ }^{1}$ QIMR Berghofer Medical Research Institute, Brisbane, QLD, Australia, ${ }^{2}$ Queensland Genomics, Brisbane, QLD, Australia, ${ }^{3}$ Brisbane Diamantina Health Partners, Brisbane, QLD, Australia, ${ }^{4}$ Queensland Genomics Community Advisory Group, Brisbane, QLD, Australia, ${ }^{5}$ Genetic Health Queensland, Royal Brisbane and Women's Hospital, Brisbane, QLD, Australia, ${ }^{6}$ Dermatology Research Centre, The University of Queensland, Brisbane, QLD, Australia and ${ }^{7}$ Health Consumers Queensland, Brisbane, QLD, Australia

Background: Through Queensland Genomics' engagement with consumers and the community, health consumers have provided anecdotal evidence which identified that a lack of understanding of precisely 'how' to access genetic testing, contributed to the consumer's diagnostic odyssey. Aim: To develop resources that will assist patients and carers in engaging with and accessing genetic and genomic health services and testing in Queensland. Methods: Community Advisory Group developed and led the creation of the resources. Drafts of the toolkit were developed by professionals with experience in genetics, health services, and communications. These drafts went through two calls for feedback, which targeted consumer and community and health stakeholders. Results: A series of five facts sheets aimed at patients and carers was developed; Introduction to genetics and genomics, Searching for a diagnosis? (accessing genetics testing in Queensland), Your guide to genetics and genomic testing, Clinical Geneticists in Queensland (public and private), and Genetics and Genomics for GPs. The toolkit has been translated into five languages other than English; Arabic, Persian (Farsi), Japanese, Vietnamese, and Chinese (simplified). A promotional campaign has been used to promote the toolkit, both online and through stakeholders' preferred communication methods (i.e. social media, newsletters). Conclusion: Engaging community and health service stakeholders in developing patient-focused resources, effectively created informative resources. Research assessing the impact of the resources on health service access and diagnosis, is needed to demonstrate the implications for patients and the value of replicating the toolkit initiative in other health jurisdictions.

\section{Supporting Families in Clinical Trials}

Jessie Head-Gray, Supriya Raj, Chloe Stutterd and Ravi Savarirayan

Skeletal Therapies Group, Murdoch Children's Research Institute, Melbourne, VIC, Australia

Background: Participation in an pediatric clinical drug trial with an invasive mode of delivery requires significant commitment from participants' families. There is limited literature to inform practice and 
ensure families are adequately supported during these trials. Aim: To understand parental experiences and the challenges of participating in a long-term pediatric clinical drug trial. Methods: A qualitative study was conducted in the form of an anonymized survey sent via a third party vendor. The survey was sent to 16 participants who had been enrolled in the drug trial for $>12$ months. Results: The most important factor influencing parental experience was trust in the trial team. The largest obstacle raised by parents was the mode of drug delivery, a daily subcutaneous injection. The children and their parents found the mode of delivery traumatic at trial commencement. By the end of the first year, families reported the injections had become part of daily routine. Parents reported participation in the trial required significant commitment from the whole family. The frequency of health-related appointments contributed to a level of fatigue affecting their experience. Parents reported functional improvements in their children, which they attributed to the trial. Families felt participation in the trial gave them hope for their child's future. Conclusions: The mode of medication administration has a significant impact on parental experience of participation. The early stages of the trial are particularly challenging and families can be supported by providing additional support including holistic care, peer support, coping strategies, and opportunity to provide feedback.

\section{Implementing Genomic Testing as Routine Care Within Pediatric Settings Using Implementation Science}

Hossai Gul ${ }^{1,2}$, Mike Field ${ }^{3}$, Ellenore M Martin ${ }^{3}$, Lucinda Murray ${ }^{3}$, Stephanie Best ${ }^{1,4}$, Janet Long ${ }^{1,4}$, Natalie Grainger ${ }^{5}$, Gayathri Parasivam ${ }^{5}$, Frances Rapport ${ }^{1}$ and Jeffrey Braithwaite ${ }^{1,4}$

${ }^{1}$ Australian Institute of Health Innovation, Macquarie University, Sydney, NSW, Australia, ${ }^{2}$ NSW Agency for Clinical Innovation, Sydney, NSW, Australia, ${ }^{3}$ Genetics of Learning Disability Service (GOLD service), Hunter Genetics, Newcastle, NSW, Australia, ${ }^{4}$ Australian Genomics, Murdoch Children's Research Institute, Melbourne, VIC, Australia and ${ }^{5}$ Centre for Genetics Education, NSW Health, Sydney, NSW, Australia

Background: Australian children with intellectual disability can access genomic testing from pediatricians via Medicare since May 2020. To support the implementation across the state of NSW, complexity science was combined with implementation science and applied through participatory action research. Aims: To (1) understand capacity within the system to perform genomic testing for intellectual disability, (2) identify barriers/facilitators to change and assess appropriateness and acceptability, (3) design implementation strategies, and 4) evaluate the impact of strategies on implementation outcomes within genetic and pediatric settings. Aims 1 and 2: Genetics: In-depth, semistructured interviews ( $n=8$ clinical geneticists/heads of department, $n=7$ genetic counselors (GC)). Pediatrics: Sequential survey $(n=102)$ followed by in-depth, semistructured interviews $(n=19)$. Aims 3 and 4: Genetics: survey of processes ( $n=8$ sites) and focus group ( $n=8$ heads of departments/ clinical geneticists, $n=6$ clinical genetics, $n=6$ GC). Pediatrics: Longitudinal in-depth, semistructured interviews $(n=12)$ with two GCs reporting on multiple sites across NSW. Results: Integrating genetic services with pediatric services to perform genomic testing requires significant behavioural changes at the individual level and process changes at the service and system levels. Genomic testing was perceived as appropriate and acceptable by genetics and pediatrics. Environmental restructuring, resources, professional roles, and boundaries were top barriers for genetics services.
Knowledge, skills, intervention complexity, and environmental context and resources were top barriers for pediatricians. Multiple strategies were applied across the system with positive impact on implementation outcomes. Conclusion: A complexity scienceinformed implementation science approach should be applied when introducing genomics into nongenetic settings.

\section{Parents' Experiences With Being Offered Additional Findings for Their Child With Hearing Loss}

D.F. Vears ${ }^{1,2}$, L Notini ${ }^{1,2}$, L Downie ${ }^{1,3}$, C Gaff ${ }^{1,3,4}$, D.J. Amor ${ }^{3}$ and J Savulescu ${ }^{5,6,7,8}$

${ }^{1}$ Murdoch Children's Research Institute, Melbourne, VIC, Australia, ${ }^{2}$ Melbourne Law School, University of Melbourne, Melbourne, VIC, Australia, ${ }^{3}$ Department of Paediatrics, University of Melbourne, Melbourne, VIC, Australia, ${ }^{4}$ Melbourne Genomics Health Alliance, Melbourne, VIC, Australia, ${ }^{5}$ Oxford Uehiro Centre for Practical Ethics, University of Oxford, Oxford, UK, ${ }^{6}$ Wellcome Centre for Ethics and Humanities, University of Oxford, Oxford, UK, ${ }^{7}$ Murdoch Children's Research Institute, Melbourne, VIC, Australia and ${ }^{8}$ Visiting Professor, Melbourne Law School, University of Melbourne, Melbourne, VIC, Australia

Background: From 2016 to 2017, 106 children diagnosed in Victoria with bilateral moderate, severe, or profound hearing loss following newborn hearing screening participated in the Melbourne Genomics Health Alliance Congenital Deafness Project. These children were offered exome sequencing to try to identify the basis of their hearing loss and were also offered additional findings. Parents could choose which types of genetic results they wished to receive: A) hearing loss, B) 'A' plus other conditions that usually develop in childhood that are preventable/treatable, C) 'A'+'B' plus some other conditions that usually develop in childhood that are not preventable/treatable. All parents received genetic counseling. Methods: We conducted semistructured interviews with parents whose children participated in the study to explore their views about being asked to choose which results to receive and experiences receiving the results. Data were analyzed using inductive content analysis. Results: Nineteen parents of 18 children were interviewed, which included parents who had opted for each of the three choices $(\mathrm{A}=4, \mathrm{~B}=7, \mathrm{C}=7)$. Two had received additional findings. Many parents found it difficult to decide which types of findings to receive, often feeling the weight of responsibility to make the 'right' decision for their child. Several parents raised concerns regarding their child's future ability to obtain insurance. Despite this, most were grateful they were offered the opportunity to choose with some feeling empowered. Conclusion: These findings will be used to refine informed consent and results in delivery processes if and when this testing is offered to children with hearing loss in the future.

\section{Perceived Heritability of Mental Health Disorders and Attitudes Towards Psychiatric Genetic Testing in Australia, the UK, and USA}

Jose J. Morosoli, ${ }^{1,2}$, Lucía Colodro-Conde ${ }^{1}$ and Sarah E. Medland ${ }^{1}$

${ }^{1}$ QIMR Berghofer Medical Research Institute, Brisbane, QLD, Australia and ${ }^{2}$ University of Queensland, Brisbane, QLD, Australia

Background: Accurate knowledge about heritability is a desired outcome of genetic education campaigns: overestimation of heritability is considered an indicator of genetic deterministic thinking and is associated with negative outcomes such as stigma and discrimination. Underestimation of heritability may indicate motivated rejection of genetics. The relationship between perceived heritability of 
mental health disorders and attitudes towards genetic testing for those conditions is unknown. Aim: To provide information about intuitive heritability and attitudes towards psychiatric genetic testing in alcohol dependence, schizophrenia, and depression, in a large sample of members of the general public from three different countries. Also, to evaluate the relationship between perceived heritability and attitudes. Methods: We surveyed 4,975 members of the general public from Australia, the UK, and the USA, and evaluated attitudes towards psychiatric genetic testing. Participants were asked about their interest in psychiatric genetic testing, perceived usefulness of genetic testing, and beliefs about malleability of behavior, among others. We also asked them to estimate the heritability of alcohol dependence, schizophrenia, and major depression. We used logistic regression to estimate the association between genetic literacy, intuitive estimates of heritability, and sociodemographic variables, on the one hand, and attitudes, on the other. Results: We found a high interest in psychiatric genetic testing. There was also a nontrivial percentage of participants with serious concerns related to learning about personal genetic predisposition. We found a significant association between most attitudes and perceived heritability. Conclusion: The association between attitudes and intuitive heritability highlights the complexity of learning about genetics.

\section{GENETIC COUNSELING}

\section{Expanded Carrier Testing at Eugene: Results of the First 1044 Tests}

Zoë Milgrom ${ }^{1}$, Emma Harrison ${ }^{1}$, Stephanie Groube ${ }^{1}$, Rachel Pope-Couston ${ }^{1}$, Ellie Greenberg ${ }^{1}$, Sarah Leighton ${ }^{1}$ and David J. Amor ${ }^{1,2,3}$

${ }^{1}$ Eugene Labs, Melbourne, VIC, Australia, ${ }^{2}$ Melbourne University, Melbourne, VIC, Australia and ${ }^{3}$ Murdoch Childrens Research Institute, Melbourne, VIC, Australia

Background: The use of expanded genetic carrier screening is increasing, but information about genetic test and counseling outcomes is lacking, particularly in the setting of couples' testing. Methods: Here, we describe our experience of offering expanded genetic carrier screening for 301 autosomal recessive and X-linked disorders, utilizing the Eugene digital genetic counseling platform and the Invitae genetic carrier screening panel. Results: Screening was performed in 1044 individuals. Carriers were identified for $224 / 301(74 \%)$ of the genes tested. Individuals tested were carriers of between 0 and 8 conditions (mean 1.8) with 787 (85\%) being carriers of 1 or more conditions. The highest carrier frequencies were for the genes HFE, CFTR, SerpinA, GALT, and BTD, although these included many low penetrance variants that were not actionable in a reproductive setting. $95 \%$ of individuals were tested as part of couples' testing, and 498 couples reports were issued, of which 24 (4.8\%) were reported as high risk of an autosomal recessive or Xlinked condition that was potentially actionable. An additional 71 couples (14\%) received increased chance reports for conditions that were assessed as not medically actionable during pregnancy, including 39 for hemochromatosis. 30 (2.9\%) of individuals received secondary findings of potential relevance to their own health, most commonly hemochromatosis. Conclusion: Expanded carrier testing identifies about 1 in every 20 couples at being at increased risk for a severe autosomal recessive or X-linked disorder. Careful genetic counseling and assessment of couples' results are required to ensure accurate communication of disorder severity and level of risk.

\section{Exploring Training Needs of New Genetic Counselors in Their Transition from Intake Assistant Roles}

Jo Martinussen ${ }^{1}$, Katrina Monohan ${ }^{1}$, Linda Cicciarelli ${ }^{1}$ and Maira Kentwell ${ }^{1,2,3}$

${ }^{1}$ Parkville Familial Cancer Centre and Genomic Medicine, Melbourne, VIC, Australia, ²Department of Oncology and Dysplasia, The Royal Women's Hospital, Melbourne, VIC, Australia and ${ }^{3}$ Department of Medicine, University of Melbourne, Melbourne, VIC, Australia

Intake assistants are an increasingly common role seen in many clinical genetic services. Students enrolled in a Master of Genetic Counseling course have routinely been employed as intake assistants for many years at the Parkville Familial Cancer Centre and Genomic Medicine Department (PFCCGM) undertaking tasks and responsibilities aligned with the Australasian Society of Genetic Counsellors Position Statement for Intake Assistants. This group of genetic counselors have a unique transition pathway from a student working within a genetics service to genetic counselor. To better understand the education, training, and support needs of graduate genetic counselors during their transition period from intake assistant to genetic counselor, an anonymous mixed methods survey will be administered to eligible participants. Participants will include genetic counselors currently employed at the PFCCGM who have previously been employed as an intake assistant. We anticipate results will identify the training and support needs of those transitioning from intake assistant to genetic counselor. We will present the benefits and challenges of transitioning from intake assistant to genetic counselor. This data will be utilized to structure a training and on-boarding programme that pre-emptively meets the needs identified. This survey may also prove a useful foundation for future research into the support needs of new genetic counselors.

\section{How Do Translocation Carriers and Their Partners Experience Noninvasive Prenatal Testing to Detect Unbalanced Translocations?}

Marta Cifuentes Ochoa, Alison D. Archibald, Nicola J. Flowers and Mark D. Pertile

Victorian Clinical Genetics Services, Melbourne, VIC, Australia

Background: Carriers for balanced chromosome translocations have an increased chance of passing an unbalanced translocation to their children. Little is known about how couples adapt to this information and make reproductive decisions. Victorian Clinical Genetic Services has offered noninvasive prenatal testing (NIPT) to carriers of balanced reciprocal translocations since 2015. Aim: To explore the experience of couples having NIPT to detect unbalanced translocations and to understand their motivations for choosing this option. Methods: Semistructured interviews were conducted with translocation carriers and their partners. Purposive sampling based on the participant's geographic location, requesting practitioner speciality, and NIPT result was used to capture a range of experiences. Interviews covered the impact of being identified as a translocation carrier, motivation for accepting testing, and perceived benefits and risks. Interview transcripts were analyzed using thematic analysis until consensus in coding was reached. Results: Thirteen people from 10 couples took part. Participants welcomed NIPT to screen their pregnancy for an unbalanced translocation, felt they had benefited from speaking to an expert about their translocation and recalled having sufficient confidence in the result to decline a diagnostic testing procedure. Participants had diverse experiences accessing cytogenetic testing for their children for carrier status of the reciprocal 
translocation (information not available from NIPT). Conclusions: The complex psychosocial impacts of carrying a balanced translocation highlight the importance of access to genetic counseling. Accurate assessment of reproductive risk and information about the benefits and limitations associated with NIPT should be provided to ensure couples are well supported.

\section{The Complexity Around Mosaic Embryo Decision Making - A Genetic Counseling Perspective}

Maddie Teed, Abbie Jedwab, Chloe Stutterd, Kelli Sorby and Alice Weeks

Number 1 Fertility, Melbourne, Australia

Background: Our genetic counseling team have reported mosaic results to $>250$ patients since the beginning of 2020, and there is an upward trend in patient interest, and willingness to consider transfer. As patients continue to request the transfer of mosaic embryos, and research advances regarding transfer outcomes, it is critical the genetic/medical professionals who counsel these patients are aware of the complex factors that feed into important decision making. Aim: To explore genetic counseling perspectives on counseling issues for patients considering transfer of a mosaic embryo, including barriers, commonalities, and themes that impact their decisions. Methods: Fundamental thematic analysis on genetic counseling consultation notes detailing questions, concerns, motivations, and decision outcomes was utilized for gaining a preliminary perspective. Specific case vignettes help highlight the complexity of these decisions. Results: Depending on the result, patients with PGT-A NGS reported mosaic embryo(s) have the option to consider a transfer, retaining, or discarding these embryos. Thematic analysis revealed themes regarding factors that influence decision-making. Patients less likely to proceed with embryo transfer: highly risk averse, poor management of uncertainty, perceived limited options, lived experience of unhealthy pregnancy or birth (i.e., family/friend), complex reproductive history, and high emotional burden of the decision. Patients more likely to proceed with transfer: extensive fertility journey, high level of information comprehension, encouraging community support, low emotional burden of the decision, and awareness of age-related limitations (ie. maternal age-related aneuploidy). Conclusion: Our work highlights the potential benefits of a genetic counseling guideline of factors to present/challenge to patients.

\section{The Role of the Genetic Counselor in Pediatrician Ordered Genomic Testing}

Ellenore M. Martin ${ }^{1}$, Lucinda Murray ${ }^{1}$, Mike Field ${ }^{1}$ and Hossai Gul ${ }^{2,3}$

${ }^{1}$ Genetics of Learning Disability Service (GOLD service), Hunter Genetics, Newcastle, NSW, Australia, ${ }^{2}$ Australian Institute of Health Innovation, Macquarie University, Sydney, NSW, Australia and ${ }^{3}$ NSW Agency for Clinical Innovation, Sydney, NSW, Australia

Background: In Australia, Medicare item numbers for genomic testing were introduced for childhood syndromes/intellectual disability in May 2020. These allow pediatricians to order genomic tests for eligible pediatric patients in consultation with a clinical geneticist. Aim: To illustrate how the unique skillset of the genetic counselor is essential in both supporting pediatricians to arrange genomic testing and ensuring patient care in a changing health landscape. Methods: Two genetic counselors from the Genetics of Learning Disability service used the express model reinforce change approach to facilitate pediatrician ordered genomic testing across multiple sites in NSW. This involved education provision to pediatricians through a series of webinars, demonstration of pre and post-test genetic counseling in joint clinics, and reinforcement of behavior change by providing ad hoc clinical support as pediatricians moved to independently order this testing. Both genetic counselors participated in fortnightly interviews to record their experiences with this process, including barriers and facilitators. Results: Genetic counselors can mitigate the barriers to the successful uptake of pediatrician ordered genomic testing, resulting in pediatricians independently ordering tests across multiple sites. Genetic counselors can increase pediatrician capability in genomic knowledge (e.g., consent/ counseling skills) via a variety of formats, assist in navigating the logistics of sample collection and testing, and aid in the provision of information to patients and families. Conclusion: As genomic technologies continue to evolve and Australian health policy promotes ordering of genomic tests by nongenetics health professionals, genetic counselors can play a key role in the safe, and equitable adoption and delivery of this testing.

\section{Exploring the Journey to Genetic Services: A Qualitative Study of Parental Perspectives of Children With Rare Disease}

Molly Krause ${ }^{1,2}$, Courtney K. Wallingford ${ }^{3,4, \star}$, Heather Renton ${ }^{5}$, Tatiane Yanes ${ }^{3}$, Chris Jacobs ${ }^{4}$, Gemma Brett ${ }^{6,7}$ and Aideen Mclnerney-Leo ${ }^{3}$

${ }^{1}$ Genetics Clinic, Monash Health, Monash Medical Centre, Clayton, VIC, Australia, ${ }^{2}$ Graduate School of Health, The University of Melbourne, ${ }^{3}$ The University of Queensland Diamantina Institute, University of Queensland, Dermatology Research Centre, Brisbane, QLD, Australia, ${ }^{4}$ Graduate School of Health, University of Technology Sydney, Sydney, NSW, Australia, ${ }^{5}$ Syndromes Without A Name (SWAN) Australia, ${ }^{6}$ Victorian Clinical Genetics Services, Murdoch Children's Research Institute, Royal Children's Hospital, Flemington Road, Parkville, VIC, 3052, Australia and ${ }^{7}$ Melbourne Genomics Health Alliance, Melbourne, Australia

Background: Approximately $80 \%$ of rare diseases are thought to be genetic in etiology. Individuals with these conditions often experience extended diagnostic delays, typically associated with heavy financial and psychological costs. Uptake of genetic services is limited, and little is known about the barriers and facilitators to service access in Australia. Understanding the experience of the diagnostic journey for rare disease is essential to improve and facilitate access to genetic services. Aims: Explore the current experience of the diagnostic journey for parents of children with rare disease in Queensland and Victoria. Methods: Parents of children with rare disease were recruited through the support group, Syndromes Without A Name. Semistructured interviews explored the experience of the diagnostic journey. Interviews were transcribed verbatim and thematically analyzed. Results: Twenty-four interviews were conducted, and four main themes were developed: (1) The value of diagnosis (2) Uncertainty permeates all aspects of the diagnostic journey, including post-diagnosis (3) Parents became advocates, experts, and care co-ordinators for their child out of necessity (4) Parental concerns were not taken seriously and parental expertise often unacknowledged. When asked about recommendations for improving the diagnostic experience, parents described referral guidelines, educational tools, and improved care coordination. Moreover, throughout interviews participants emphasized the importance of forming partnerships between parents and healthcare professionals (HCPs). Conclusions: Our findings highlight areas for improving the diagnostic journey for rare disease. We also emphasize the need for educational resources to support parent and HCP awareness of and access to genetic services for children with rare disease. 


\section{Atypical Results from Genomewide NIPT for a Carrier of a Balanced Translocation}

C Dao ${ }^{1}$, N Flowers ${ }^{2}$ and J Kelley ${ }^{1}$

${ }^{1}$ Mercy Hospital for Women, Melbourne, VIC, Australia and ${ }^{2}$ Victorian Clinical Genetics Service, Melbourne, VIC, Australia

Background: Noninvasive prenatal screening (NIPT) is considered a superior primary prenatal screen for common aneuploidy. For balanced translocation carriers, genomewide NIPT (gw-NIPT) is a clinically relevant and practical option. As gw-NIPT becomes integrated into routine practice and broadens in scope, it is important to remain aware of limitations possible complex results and the continued relevance of prenatal diagnosis. Aim: Our service offers gw-NIPT to balanced translocation carriers who meet certain criteria. We present a case study detailing unexpected results and subsequent counseling, investigations, and outcomes. Results: A gw-NIPT result for a carrier of a balanced translocation indicated trisomy 6 . This was suggested to have arisen from malsegregation of a maternal translocation. Viability at 11 weeks and normal obstetric ultrasounds at 12 and 16 weeks suggested confined placental mosaicism arising from a post-zygotic rescue. Microarray from amniocentesis indicated no chromosomal imbalance, although detected maternal UPD 6 and regions of isodisomy. Ongoing obstetric management was arranged in the context of intra-uterine growth restriction. Multiple genetic counseling interactions were required to facilitate communication and comprehension of these complex genetic concepts, and counseling issues adapted alongside changing concerns. Conclusion: For balanced translocation carriers, gw-NIPT has practical clinical utility. However, complex gw-NIPT results often require ongoing consultation between genetics services, laboratory, and high-risk obstetrics. Awareness of this, as well as the biological limitations which constrain gw-NIPT mean that ultrasound and prenatal diagnosis remain important investigative tools.

\section{'We're All Different. But We're All The Same' — Listening to Caregivers' Voices When Designing a Rare Genetic Epilepsy Family Conference}

Ella West ${ }^{1}$, Rebecca Macintosh ${ }^{2}$, Suzanne Nevin ${ }^{2,3}$, Erin Beavis ${ }^{2}$, Gemma McErlean $^{1}$ Alison McEwen ${ }^{1}$ and Elizabeth Emma Palmer ${ }^{2,3}$

${ }^{1}$ University of Technology Sydney, Graduate School of Health, Sydney, NSW, Australia, ${ }^{2}$ Sydney Children's Hospitals Network, Sydney, NSW, Australia and ${ }^{3}$ School of Women's and Children's Health, UNSW, Sydney, NSW, Australia.

This study aimed to explore the preferences of parents of children with rare genetic epilepsies (RGEs) for a genetic counselor organized family conference. RGEs are typically severe and complex conditions which create significant psychosocial challenges not only for the child, but the whole family. Parents of children with RGE have a wide range of support and information needs. It has been suggested that family conferences are one approach to meet these needs while potentially empowering parents and reducing psychological stress. However, little is known about parents' preferences with regards to family conference design and content. A qualitative approach using semistructured focus group interviews was used to explore the preferences and opinions of nine parents of children with RGE attending Sydney Children's Hospitals Network regarding the planned 2021 RGE family conference. Findings revealed that parents collectively shared a strong desire to network in-person with other parents of children with RGE, especially with those whose children were of similar ages. Thematic analysis highlighted a need to normalize the unique form of grief associated with caring for a child with a rare genetic epilepsy. Preferences for content included hearing other families' journeys, navigating government systems, and learning about RGE therapies and advancing clinical research. This study has informed the structure of our upcoming parent-centered family conference. It provides insight into the wide-ranging psychosocial needs of parents' of children with rare genetic epilepsies. Findings highlight that RGE parents value the importance of face-to-face networking with peers, even in the pandemic era.

\section{A Survey of Patient Satisfaction with a Clinical Genetics Service}

Sangavi Sivagnanasundram ${ }^{1,3}$, Rubi Ramzan ${ }^{1,3}$, Robert Sharrock ${ }^{1}$, Lisette Curnow ${ }^{1,2,3}$, Ivan Macciocca ${ }^{1,2}$, David Blake ${ }^{1}$ and Martin Delatycki ${ }^{1,2,3}$

${ }^{1}$ Victorian Clinical Genetics Service, Melbourne, VIC, Australia, ${ }^{2}$ Murdoch Children's Research Institute, Melbourne, VIC, Australia and ${ }^{3}$ University of Melbourne, Melbourne, VIC, Australia

Background: Although there is no consensus on exactly what or how to measure patient satisfaction, it has become an integral part of assessing health care delivery. National standards state that health services monitor patient satisfaction and use the outcomes to set realistic goals for improving partnerships between clinicians and patients. Aim: To develop and administer a purpose-designed survey to assess patient satisfaction of patients who attended a Victorian Clinical Genetics Services (VCGS) clinic and identify areas for service improvement. Method: The survey was developed following a review of existing patient satisfaction surveys in Australia. The final survey contained questions from the Australian Hospital Patient Experience Question Set which were adapted for outpatient use, the Genomics Outcome Scale and a series of purpose-designed service-specific questions. An online survey was developed using the RedCap survey tool which was piloted on 12 patients. The survey was sent by email to all patients seen at a VCGS Royal Children's Hospital or regional clinic during February and March 2021. Results: 606 surveys were sent, with 89 returned to date (an initial response rate of $14.7 \%$ ). $93 \%$ of respondents were parents of a patient, with the remainder being patients themselves. Preliminary analysis of the current data shows that approximately $50 \%$ of appointments were by telehealth, $93.4 \%$ of patients rate the overall quality of care as good or very good, and $89 \%$ would recommend the service to a friend. Data collection is ongoing, and results from the final dataset will be presented.

\section{The Experiences and Needs of Individuals With MNF And FTD, and Their Family Members, Towards Genetic Counseling and Genetic Testing}

Ashley Crook ${ }^{1,3}$, Chris Jacobs ${ }^{1}$, Toby Newton-John ${ }^{1}$ and Alison McEwen ${ }^{1}$

${ }^{1}$ University of Technology Sydney, Graduate School of Health, Sydney, NSW, Australia, ${ }^{2}$ Centre for MND Research, Department of Biomedical Science, Faculty of Medicine and Health Sciences, Macquarie University, Sydney, NSW, Australia and ${ }^{3}$ Department of Clinical Medicine, Faculty of Medicine and Health Sciences, Macquarie University, Sydney, NSW, Australia

Background: Genetic counseling and diagnostic genetic testing is becoming increasingly routine in motor neuron disease (MND) and/or frontotemporal dementia (FTD) care due to the emergence of genotype-driven therapy trials. There are currently no consistent, evidence-based genetic counseling approaches to diagnostic testing in MND/FTD. Aim: Explore patient and family members' diagnostic testing and counseling experiences and generate recommendations to inform care in future. Methods: Interpretive description 
methodology was used. Semistructured interviews were conducted with MND/FTD patients and family members either by audio or text, one-on-one or conjoint (depending on participant preference). Advertising occurred through Australian MND and FTD organizations. Themes were developed using inductive thematic analysis. Results: Eighteen individuals from 13 families participated. Diagnostic testing occurred alongside other MND/FTD disease burdens due to progressing disease. Testing pathways varied and were informed by local health system characteristics; some were barriers to a positive genetic counseling experience. Interactions with health professionals and MND/FTD association staff that included open communication, time to facilitate adjustment, and address questions led clients to feel supported and informed regarding testing and sharing information with other family members. Individual and family characteristics informed the level of information and support required. Conclusion: A supportive, informative genetic counseling process adapted to the client's unique needs must be facilitated, and testing pathways must be clarified and consistent. Guidelines to inform best practice are likely to be beneficial. These improvements could reduce some of the burdens of the disease lived experience by improving access to and quality of genetic counseling provided across Australia.

\section{Understanding the Introduction and Evolution of Percept Noninvasive Prenatal Testing Results in Victoria}

Emma Murdoch ${ }^{1,2}$, Ruth Leibowitz ${ }^{3}$, Anaita Kanga-Parabia ${ }^{1}$, Lisa Hui ${ }^{3,4,5}$ and Alison Archibald ${ }^{1}$

${ }^{1}$ Victorian Clinical Genetics Service, Melbourne, VIC, Australia, ${ }^{2}$ Department of Paediatrics, University of Melbourne, VIC, Australia, ${ }^{3}$ Murdoch Children's Research Institute, Melbourne, VIC, Australia, ${ }^{4}$ Mercy Hospital For Women, Melbourne, VIC, Australia and ${ }^{5}$ Department of Obstetrics and Gynaecology, University of Melbourne, VIC, Australia

Background: Since the introduction of noninvasive prenatal testing (NIPT), there has been rapid evolution in clinical practice and an increase in the range of conditions which can be detected beyond the common autosomal trisomies and sex chromosome aneuploidy. Aims: This study aimed to understand the changes in NIPT since its introduction through exploring healthcare provider (HCP) experiences and one NIPT laboratory's testing data. Methods: A mixedmethod approach was used. (1) Key-informant interviews were undertaken with HCPs with at least five years' general NIPT experience. Interviews were transcribed verbatim and analyzed using inductive content analysis. (2) A clinical audit of Percept (Victorian Clinical Genetics Services branded NIPT) with descriptive analysis of result types (2015-2020). Results: To date, five HCPs have been interviewed; genetic counselors (2), clinical geneticist (1), obstetrician (1), general practitioner (1). HCPs described workload and role changes since NIPT's introduction to clinical practice due to increasingly complex NIPT results. Counseling patients required longer appointments, more preparation, and involvement by the genetics HCP. Analysis of 74,550 records for Victorian women who had Percept NIPT supported reported HCP experiences of NIPT, with complex results (rare aneuploidy, unbalanced translocations, and segmental imbalances) forming an increasing portion of results: $0.4 \%$ (2015) to $1.1 \%$ (2019).

Discussion: Whilst NIPT has improved prenatal screening for chromosome conditions, leading to a greater number and range of severe conditions identified, HCPs require greater resourcing and support for changing caseloads due to complex NIPT results

\section{Scenario-Based Responses to Hypothetical Fetal Diagnosis By Noninvasive Genetic Testing}

Sarah Long ${ }^{1,2}$, Peter O'Leary ${ }^{2,3}$ and Jan E. Dickinson ${ }^{2}$

${ }^{1}$ Genetic Services of Western Australia, King Edward Memorial Hospital for Women, Perth, WA, Australia, ${ }^{2}$ Division of Obstetrics and Gynaecology, The University of Western Australia, Perth, WA, Australia and ${ }^{3}$ PathWest Laboratory Medicine, QE2 Medical Centre, Perth, WA, Australia

Background: Cell-free fetal DNA (cfDNA) is now a commercially available test which has been used in noninvasive prenatal testing (NIPT) to detect single gene disorders. What women would do with this information on a population screening level (in a scenario where a person had no lived experience of these conditions from a known family history) is yet to be explored. Aim: To discuss several genetic conditions that could be detected with single gene NIPT with women to explore attitudes toward termination of pregnancy for these conditions. Methods: Several genetic conditions were described without being named, including neurofibromatosis 1 (NF1), Duchenne muscular dystrophy (DMD), Hereditary breast and ovarian cancer syndrome (HBOC), 22q11 deletion syndrome, Turner (XO) syndrome, and Huntington's disease (HD). Scenarios such as a healthy carrier and an incidental finding were also included. An online survey was carried out with 219 female participants from Western Australia. The scenarios were not named to avoid any preconceived ideas about the conditions. Results: The highest response for whether to end a pregnancy at gestation up to 18 weeks was for the DMD scenario described, followed by HD, 22q11 and then NF1. A healthy carrier, Turner syndrome, incidental findings, and HBOC had relatively low rates of women agreeing they would end a pregnancy, but there was still a proportion of women who would end a pregnancy in these circumstances. Conclusion: Screening a population for rare genetic disorders would be expected to increase the termination rate and require widespread genetic counseling.

\section{GENETIC EDUCATION}

\section{Development of Decision Aids to Support Mainstreaming of Genomic Testing in Renal Medicine}

Lindsay Fowles1'2, Mia McLanders ${ }^{3}$, Raden Sucalit ${ }^{3}$, Andrew Mallett1'4 and Chirag Patel1'2

${ }^{1}$ The Genomic Institute, Metro North Hospital and Health Service, Brisbane, QLD, Australia, ${ }^{2}$ Genetic Health Queensland, Royal Brisbane \& Women's Hospital, Brisbane, QLD, Australia, ${ }^{3}$ Clinical Skills Development Service, Metro North HHS, Brisbane, QLD, Australia and ${ }^{4}$ Department of Renal Medicine, Townsville University Hospital, Townsville, QLD, Australia

Background: As technologies advance, knowledge grows, and costs decrease, genomic testing is increasing in value as a diagnostic tool for patients, including those with kidney disease. Mainstreaming of genomic testing brings value to the patient as they receive their testing from their primary clinician early in their diagnostic journey. However, there is evidence that nongenetic specialists have limited proficiency and confidence in selecting, ordering, and interpreting genomic tests. Clinical decision aids can support the nongenetic specialist in these processes. Aim: Develop clinical decision aids to 
support the adoption of genomic testing as a diagnostic tool in renal services across Queensland. Methods: A project team comprising a nephrologist (AM), clinical geneticist (CP), and a genetic counselor (LF) developed content for a series of decision aids. Content was adjusted based on potential user feedback. An iterative review process aligned the material with human factors and graphic design principles. Results: Decision aids to support mainstreaming of genomic testing for genetic kidney disease were developed. Topics included: identification of potential genetic kidney disease patients; key clinical diagnostic features for the different genetic kidney disease subtypes; family history queries; identification of appropriate genomic test panels; processes for requesting tests; and an overview of result interpretation. Contact details for an MDT where expert support can be accessed were provided. Conclusion: This series of decision aids were positively reviewed by renal specialists and are expected to support mainstreaming of diagnostic genomic testing in renal medicine. These aids could guide the development of similar aids for other clinical specialties.

\section{GENETIC EPIDEMIOLOGY}

\section{The Epidemiology of Hereditary Pancreatitis in South Australia}

Denghao $\mathrm{Wu}^{1}$, Tristan Bampton ${ }^{2}$, Lyle Palmer ${ }^{1}$, Hamish Scott ${ }^{3}$, Richard Couper ${ }^{4}$ and Toby Coates $^{2}$

${ }^{1}$ The University of Adelaide, SA, Australia, ${ }^{2}$ Royal Adelaide Hospital, SA, Australia, ${ }^{3}$ SA Pathology, SA, Australia and ${ }^{4}$ Women's and Children's Hospital Adelaide, SA, Australia

Background: Hereditary pancreatitis (HP) is a debilitating condition caused by the inheritance of a variety of genetic mutations. HP results in inflammation of the pancreas from a young age, chronic abdominal pain, and dependency upon pain management opioids. Severe cases of HP are candidates for total pancreatectomy and islet auto transplant (TP-IAT) surgical treatment. Aims: This project is the first to identify Australian families suffering from HP and assess the correlation between phenotypic disease outcome and genotypic variant. Methods: Patients with HP were identified from existing hospital records and interviewed for phenotype. Salivary biosamples were obtained from patients and family members to be whole-exomesequenced (WES) and analyzed in silico using bioinformatics toolkits (GATK). Results: A total of 5 pedigrees and 4 individual probands comprising 47 individuals were recruited for the project. Four families possess the mutation PRSS1(3 family with R122H, 1 family with A86T). In total, 23 PRSS1 and 9 SPINK1 variant carriers across multiple generations were identified, 13 of which self-identified as Indigenous Australian. Our estimated prevalence of HP in South Australia is much higher than the value of $0.1-0.3 / 100,000$ previously described in European populations. Bioinformatics analyses of WES genotypic data yielded three potentially pathogenic variants identified outside of known HP-associated gene: ECE1, GJA5, and SPTBN5. Conclusions: The study described the prevalence of HP in an Australian population for the first time, highlighted the importance of utilizing genetic studies to guide medical decision-making in HP, and successfully established a patient database for candidates of TP-IAT treatment.

\section{GENOMICS}

\section{What Do the General Public Think About Clinical Genomics?}

Isabella Sherburn ${ }^{1}$, Keri Finlay ${ }^{1}$ and Stephanie Best ${ }^{1,2}$

${ }^{1}$ Australian Genomics, Melbourne, VIC, Australia and ${ }^{2}$ Australian Institute of Health Innovation, Macquarie University, Sydney, NSW, Australia

Background: Genomic testing has typically been employed in the fields of rare disease and cancer. However, as genomic sequencing becomes more readily available in mainstream healthcare, the general population is more likely to be exposed to genomics. Therefore, how the genomic uninformed public perceives clinical genomic testing is paramount. This knowledge will be essential in responding to key concerns of the public and allowing for efficient implementation of clinical genomics into wider healthcare. However, most studies have focused on the perceptions of the genomic informed. Aim: To investigate the genomic naïve publics' perceptions of clinical genomics and clinical genomic testing. Methods: MEDLINE, PubMed, and Web of Science databases were searched generating 1612 articles in total. Narrative synthesis was used to extract thematic data along a person's decision-making pathway. Results: Twelve fulltext articles were included in the final analysis. Most of the studies used questionnaires to determine attitudes of the public towards clinical genomics $(n=9)$. The US conducted the most studies $(n=4)$, followed by Japan $(n=2)$. Themes identified included: pre-exposure perceptions of clinical genomics (e.g., sources of information, influences on decision to undertake genomic testing), personal values and beliefs and implications of undertaking genomic testing, and fear of discrimination. Conclusion: The current literature surrounding the perceptions of the genomic naïve public is scarce. The studies analyzed to demonstrate the uncertainty and inconsistencies in the area and the importance of considering cultural contexts among other factors when discerning attitudes towards clinical genomic testing.

\section{Illumina Clinical Services Laboratory (ICSL) Framework Designed to Improve the Evaluation and Reporting of Incidental Findings in Clinical Genetic Tests}

Anjana Chandrasekhar ${ }^{\star}$, Carolyn M. Brown ${ }^{\star}$, Alison J. Coffey*, Erin Thorpe, Ryan J. Taft, Denise L. Perry and R. Tanner Hagelstrom

Illumina Inc, San Diego, CA, USA

Incidental findings (IFs) present challenges for laboratories and clinicians, as accepted recommendations for evaluation or reporting are lacking. Additionally, protocols for handling IFs can be subjective, time consuming, and difficult to scale. ICSL offers clinical whole genome sequencing (cWGS) with a policy of reporting IFs which may impact medical management. We have developed and validated a framework to streamline the evaluation of IFs and improve consistency in reporting. Stopping points at which an IF may be deemed reportable or not were first defined. For single nucleotide variants (SNVs) impacting single genes, the following criteria must be met: (1) the strength of the gene-disease relationship is classified as 'Strong' or 'Definitive' according to the ClinGen protocol, (2) the variants are classified as likely pathogenic (LP) or pathogenic (P), and (3) the variants are in the correct zygosity. Next, a threshold 
was set for ClinGen actionability scores to qualify IFs for reporting. Finally, if actionability scores were insufficient or unavailable, a literature search was performed to identify interventions, screening guidelines, or circumstances to be avoided by carriers. Heterozygous LP or P copy number variants (CNVs) expected to produce a phenotype, or variants of unknown significance (VUS) gains $\geq 1 \mathrm{Mb}$ and losses $\geq 500 \mathrm{~kb}$ were also reported as IFs. Several questions remain unanswered, for example, reporting of risk factors and IFs in individuals who opt out of SFs. Nevertheless, for laboratories offering cWGS, this framework will streamline analysis and bring consistency and transparency to reporting IFs.

\section{Modern-Day Reproductive Carrier Screening: The Dynamic Nature of Variant Interpretation and the Impact on Risk Interpretation and Clinical Management}

Stephanie Liew, Nicole Schonrock, Julia Wilkinson, Robert Slotnick, Sarah Poll, Asia Mitchell, Nicole Faulkner and Swaroop Aradhya

Invitae, San Francisco, CA, USA

Background: Reproductive carrier screening raises unique questions related to variant reclassifications because variants of uncertain significance (VUS) are not routinely reported in this context, and there are time constraints around pregnancy planning and management. Here, we review the extent and timing of variant reclassification in a large clinical cohort and discuss potential clinical management implications of this reclassification. Methods: Carrier screening was performed by next-generation sequencing with deletion and duplication analysis of up to 301 genes, and only pathogenic or likely pathogenic $(\mathrm{P} / \mathrm{LP})$ variants were originally reported. Clinically significant reclassifications and their timing were identified. Results: Out of 126,424 patients included in this analysis, 4,179 ( 3.3\%) had at least one clinically significant reclassification with an average time to reclassify of 286 days. In this cohort, 418 patients had partners who also underwent testing. Only four patients with a reclassification became 'at-risk' after reclassification ( 0.1\%). Conclusions: Although reclassifications occur infrequently in carrier screening, they can have significant clinical and psychosocial implications for patients and clinicians. A clear consensus on how to perform this reanalysis and provide updates to patients and clinicians is not available yet. As this issue continues to impact carrier screening, both the reporting laboratories and the clinician will have roles in communicating and managing the impacts on patient care. Additional studies are needed to continue to understand the impact on both clinicians and patients of receiving reclassified variant information and also to inform laboratory standards for reclassifying variants in reproductive carrier screening.

\section{Ontoclick: A Web Browser Extension to Facilitate Biomedical Knowledge Curation}

Anthony $\mathrm{Xu}^{1}$, Aravind Venkateswaran ${ }^{1}$ and Andreas Zank ${ }^{2,3,4}$

${ }^{1}$ School of Computer Science and Engineering, UNSW, Sydney, NSW, Australia, ${ }^{2}$ Department of Clinical Genetics, The Children's Hospital at Westmead, Sydney, NSW, Australia, ${ }^{3}$ Faculty of Medicine and Health, The University of Sydney, Sydney, NSW, Australia and ${ }^{4}$ Kinghorn Centre for Clinical Genomics, Garvan Institute of Medical Research, Sydney, NSW, Australia

The world's biomedical literature has achieved a scale that the human brain cannot possibly process. Computers could assist with searching through this vast sea of knowledge, but this requires 'knowledge curation', converting unstructured text into well-defined units of information, such as a set of Human Phenotype Ontology terms, that a computer can process. Knowledge curation can be a repetitive and laborious process but without knowledge curation, vast amounts of knowledge in the biomedical literature will remain untapped. The paucity of user-friendly tools is one of the reasons for the lack of widespread adoption of good biomedical knowledge curation practices. Here, we present Ontoclick, a web browser extension that streamlines the process of annotating a text span with a relevant ontology term, a key component of knowledge curation. We hope this tool will make biocuration more accessible to a wider audience of biomedical researchers.

\section{Spinal Muscular Atrophy Diagnosis and Carrier Screening From Whole Genome Sequencing Data}

Xiao Chen, Andrew Warren and Michael A. Eberle

Illumina, San Diego, CA, USA

Spinal muscular atrophy (SMA), caused by loss of the SMN1 gene, is a leading cause of early childhood death. The American College of Medical Genetics and Genomics recommends SMA carrier screening be offered to all couples either before conception or early in pregnancy. Due to the near identical sequences of SMN1 and SMN2, analysis of this region is challenging. We developed a bioinformatics method, SMNCopyNumberCaller, that accurately identifies the copy number (CN) of SMN1 and SMN2 using whole genome sequencing (WGS) data. We characterized SMN1 and SMN2 in 12,747 genomes, identified 1568 samples with SMN1 gains or losses and 6615 samples with SMN2 gains or losses, and calculated a pan-ethnic carrier frequency of $2 \%$, consistent with previous studies. Additionally, $99.8 \%$ of our SMN1 and $99.7 \%$ of SMN2 CN calls agreed with orthogonal methods, with a recall of $100 \%$ for SMA and $97.8 \%$ for carriers, and a precision of $100 \%$ for both SMA and carriers. By developing SMNCopyNumberCaller using multi-ethnic samples, we were able to identify higher genetic variability in Africans and exclude variable sites that cannot reliably differentiate $S M N 1$ from $S M N 2$, thus optimizing accuracy across all populations. To make SMNCopy NumberCaller useful in clinical settings where WGS is the preferred testing modality, we created a visualization tool that produces static images that represent the data to QC the calls. SMNCopyNumberCaller enables SMA testing to be offered as part of a comprehensive test in neonatal care, carrier screening, and other clinical settings where WGS may be indicated.

\section{Efficacy of First-Line Gene Panel as Screening Prior to Whole Genome Sequencing in the Diagnosis of Inborn Errors Of Immunity}

Pei Dai ${ }^{1,2}$, Karen Enthoven ${ }^{1}$, Georgina Hollway ${ }^{1,3}$, Stuart Tangye ${ }^{1}$, Paul Gray ${ }^{4}$ Melanie Wong ${ }^{4}$, Warren Kaplan ${ }^{2,5}$, Tri Giang Phan ${ }^{1,2}$, Leslie Burnett ${ }^{2,5}$ and on behalf of CIRCA

${ }^{1}$ Garvan Institute of Medical Research, Sydney, NSW, Australia, ${ }^{2}$ St Vincent's Clinical School, Faculty of Medicine, University of New South Wales, Sydney, NSW, Australia, ${ }^{3}$ University of New South Wales, Sydney, NSW, Australia, ${ }^{4}$ Sydney Children's Hospital Network, NSW, Australia and ${ }^{5}$ Kinghorn Centre for Clinical Genomics, Garvan Institute of Medical Research, Sydney, NSW, Australia

Background: Inborn errors of immunity (IEI) are rare diseases resulting from pathogenic variants in genes regulating immune function, which are curated by the International Union of Immunological Societies (IUIS). While next generation sequencing (NGS) has significantly improved the diagnosis of IEIs, it is costly and slow. Currently, there are uncertainty regarding whether patients should 
undergo initial screening with an IUIS gene panel or proceed directly to clinical exome sequencing (CES) or whole genome sequencing (WGS). Aim: To determine the clinical utility of an IUIS gene panel-first approach compared to direct CES/WGS. Method: Between 2016 and 2017, 56 suspected IEI cases from the Clinical Immunogenomics Research Consortium Australasia (CIRCA) cohort underwent first-line WGS. A likely pathogenic/pathogenic (ACMG Class 4/5) variant was found in 12/56 (21\%) of cases and variants of uncertain significance (VUS) in 5/56 (9\%) of cases. We retrospectively reviewed these 17 variants in the setting of the current existing diagnostic methodology of two leading commercial vendors offering abridged IUIS gene panels to determine if they would have been identified by a panel-first approach. Result: We found a gene panel-first approach would have identified 10/12 likely pathogenic/pathogenic variants and $3 / 5$ VUSes in panels provided by both vendors. Conclusion: First-line screening with an abridged IUIS gene panel is a cost-effective method for detecting clinically relevant variants in suspected IEIs. CES/WGS should be considered for the remaining unsolved cases. This two-tiered approach to testing may have clinical utility by providing a cost-effective way to expedite the genetic diagnosis of IEIs.

\section{ATAD3 CNVs and Severe Lethal Mitochondrial Disease: Genotype-Phenotype Correlations and Functional Genomic Strategies for Diagnosis}

Ann E. Frazier ${ }^{1,2}$, Linden S. Muellner-Wong ${ }^{3}$, Luke E. Formosa ${ }^{4}$, Rocio Rius ${ }^{1,2}$, Daniella H. Hock ${ }^{3}$, Alison G. Compton ${ }^{1,2}$, Susan M. White ${ }^{1,2,5}$, Joy Yaplito-Lee ${ }^{6}$, Heidi Peters ${ }^{6}$, Min Wang ${ }^{1}$, André E. Minoche ${ }^{7}$, Mark J. Cowley ${ }^{7,8,9}$, Michael T. Ryan ${ }^{4}$, Cas Simons ${ }^{1}$, John Christodoulou ${ }^{1,2,5,10}$, David A. Stroud ${ }^{3}$ and David R. Thorburn ${ }^{1,2,5}$

${ }^{1}$ Murdoch Children's Research Institute, Royal Children's Hospital, Melbourne, VIC, Australia, ${ }^{2}$ Department of Paediatrics, University of Melbourne, Melbourne, VIC, Australia, ${ }^{3}$ Department of Biochemistry and Pharmacology and Bio21 Molecular Science and Biotechnology Institute, University of Melbourne, Melbourne, VIC, Australia, ${ }^{4}$ Department of Biochemistry and Molecular Biology, Monash Biomedicine Discovery Institute, Monash University, Melbourne, VIC, Australia, ${ }^{5}$ Victorian Clinical Genetics Services, Murdoch Children's Research Institute, Royal Children's Hospital, Melbourne, VIC, Australia, ${ }^{6}$ Department of Metabolic Medicine, Royal Children's Hospital, Melbourne, VIC, Australia, ${ }^{7}$ Kinghorn Centre for Clinical Genomics, Garvan Institute of Medical Research, Sydney, NSW, Australia, ${ }^{8}$ Children's Cancer Institute, Sydney, NSW, Australia, ${ }^{9}$ St. Vincent's Clinical School, University of New South Wales, Sydney, NSW, Australia and ${ }^{10}$ Disciplines of Genomic Medicine and Child and Adolescent Health, Sydney Medical School, University of Sydney, NSW, Australia

Background: The ATAD3 locus comprises three highly homologous genes ( $A T A D 3 C, A T A D 3 B, A T A D 3 A$ ) encoding mitochondrial proteins implicated in cholesterol metabolism, mitochondrial DNA homeostasis, and morphology. Recessive deletions or dominant duplications cause lethal mitochondrial disorders characterized by pontocerebellar hypoplasia or cardiomyopathy, respectively. In our experience, the ATAD3 locus has emerged as the most common cause of lethal perinatal mitochondrial disease, but the repetitive sequence at the locus means diagnoses may be missed or misinterpreted using current genomic strategies. Aims: To evaluate genotype-phenotype correlations and techniques for screening ATAD3 variants by characterizing molecular rearrangements in patients with novel ATAD3 CNVs. Methods: Whole genome and exome sequencing; long read sequencing (ONT); microarrays; RNA studies; quantitative proteomics; biochemical analyses. Results: We recently identified 17 individuals (16 families) with a lethal neonatal presentation including cardiomyopathy, lactic acidemia, and corneal clouding/cataracts due to recurrent de novo $68 \mathrm{~Kb} A T A D 3$ duplications (Frazier et al., $2021 \mathrm{Med}, 2: 49-73$ ). The duplications all generated identical chimeric ATAD3A/ATAD3C proteins, which appeared to act dominantly, altering ATAD3 complexes and mitochondrial complex I activity in heart. Our genomics investigations have since uncovered additional subjects with novel ATAD3 CNVs. These include benign biallelic deletions, along with recurrent $A T A D 3$ duplications plus a second $A T A D 3 \mathrm{CNV}$ that can ameliorate the severe lethal presentation. Functional screens to evaluate ATAD3 expression (quantitative proteomics) and ATAD3 complex assembly (blue native-PAGE) showed promising results in validating pathogenic variants. Conclusion: Novel ATAD3 CNVs require extensive functional studies to unravel the complex molecular changes and contribution to pathogenicity, with several promising strategies emerging.

\section{Pharmacogenomic Testing: Perception of Clinical Utility, Enablers and Barriers to Adoption in Australian Hospital Settings}

Angela Pearce ${ }^{1}$, Bronwyn Terrill ${ }^{1,2}$, Sid Patanwala ${ }^{3,4}$, Jan-Willem Alffenaar ${ }^{3,5}$, Sarah Kummerfeld ${ }^{1,2}$, Richard Day ${ }^{2,6}$, Mary-Anne Young ${ }^{1,2}$ and Sophie Stocker ${ }^{2,3,6}$

${ }^{1}$ Kinghorn Centre for Clinical Genomics, Garvan Institute of Medical Research, Darlinghurst, Sydney, Australia, ${ }^{2}$ St Vincent's Clinical School, Faculty of Medicine, The University of New South Wales, Sydney, Australia, ${ }^{3}$ Sydney Pharmacy School, Faculty of Medicine \& Health, The University of Sydney, Sydney, Australia, ${ }^{4}$ Royal Prince Alfred Hospital, Sydney, Australia, ${ }^{5}$ Westmead Hospital, Sydney, Australia and ${ }^{6}$ Department of Clinical Pharmacology \& Toxicology, St Vincent's Hospital, Sydney, Australia

Background: Pharmacogenomic tests can be used to detect variants that affect drug metabolism and predict patients' responses to medications. Despite anticipated benefits to patient care, implementation of pharmacogenomic testing in Australia is limited. Aim: This study aimed to assess health professionals' perceptions of pharmacogenomic testing, including attitudes to testing, and potential barriers or enablers, in three Australian hospital settings. Methods: An online survey (10-12 mins, 31 multiple choice and short answer questions) was adapted from published measures to assess health professionals' usage, knowledge, confidence, and experience with pharmacogenomic testing, and their perceptions of benefits, risks, and barriers to its implementation. Results: Health professionals involved in prescribing decisions across three NSW hospitals ( $N=103$; $70 \%$ physicians; $23 \%$ pharmacists) completed the survey between July 2020 and May 2021. Although most health professionals (79\%) thought pharmacogenomic testing had clinical utility and relevance to their practice, only $33 \%$ had used or intended to use pharmacogenomic testing to guide prescribing decisions. A primary barrier to use was a lack of knowledge about pharmacogenomic testing, including when and how to test and the interpretation of results. Absence of guidelines and a perceived scarcity of accessible evidence-based information were additional barriers. Few (47\%) respondents reported receiving education in pharmacogenomics. Guidance from institutional experts was perceived to increase the likelihood of using pharmacogenomic testing to inform prescribing decisions in future.

Conclusion: Further education, development of guidelines, and onsite expert advice could help improve the implementation and adoption of pharmacogenomic testing into routine clinical care to inform prescribing decisions. 


\section{The Utility of RNA-Sequencing in the Study of Muscle Biology and Neuromuscular Disease}

Andrei Smolnikov, Emily Oates and Marc Wilkins

School of Biotechnology and Biomolecular Sciences, UNSW Sydney, Australia

Background and Aims: RNA sequencing is a powerful massively parallel sequencing (MPS)-based technology used to interrogate the impacts of likely pathogenic mutations and identify potentially diagnostically useful abnormal transcripts in the rare disease setting. Our study aimed to assess the strengths and limitations of RNA-seq in the context of neuromuscular disease diagnostic work and research aimed at furthering our understanding of fundamental muscle biology. Methods and results: Mapping and coverage analyses of new and existing control short read foetal and mature muscle RNA-seq data sets and in silico modeling showed that a significant number of clinically relevant genes contained regions with far from optimal sequencing coverage due to poor mappability using current short-read methodologies, potentially resulting in missed diagnostic opportunities. We also found strong positional bias in short-read RNA-seq datasets and demonstrated that clinically relevant genes were disproportionately impacted by this bias, with a variety of effects on downstream calculations. In addition, a Nanopore (long read) sequencing study of mouse striated muscle showed no evidence of positional bias in reads mapping to mouse genes. Further optimization in mouse muscle and extension into direct (native) long read RNA-seq are ongoing. Conclusion: Overall, our study has provided novel insights into fundamental muscle biology and the clinical utility of muscle RNA-seq, affirming established and emerging RNA-seq technologies as powerful tools in the study of neuromuscular disease.

\section{Pathogenic Variants in Hereditary Cancer Syndrome Genes are Prevalent Among Breast Cancer Patients Not Meeting Various International Genetic Testing Guidelines}

Nicole Schonrock ${ }^{1}$, Sarah M. Nielsen ${ }^{1}$, Emily Decker $^{1}$, Natalie Rickers $^{1}$, Alekhya Narravula $^{1}$ Peter Beitsch ${ }^{2}$, Pat Whitworth ${ }^{3}$, Edward D. Esplin ${ }^{1}$ and Robert L. Nussbaum ${ }^{1}$

${ }^{1}$ Invitae, San Francisco, CA, USA, ${ }^{2}$ Dallas Surgical Group, Dallas, TX, USA. and

${ }^{3}$ Nashville Breast Center, Nashville, TN, USA

Background: Clinical management options have expanded for patients harboring pathogenic variants (PVs) in cancer predisposition genes. Historically, testing costs and clinical implementation challenges led to restrictive testing guidelines in many countries. Increasing evidence demonstrates that broader testing is a cost-effective way to identify patients with PVs. We assessed the efficacy of multiple international testing guidelines in identifying breast cancer (BC) patients with clinically actionable PVs. Methods: We reanalyzed a prospective cohort of U.S.-based, primarily Northern European, BC patients, referred for germline genetic testing (PMID: 30526229). We applied testing guidelines from Australia, UK, and two Canadian provinces (Ontario, British Columbia) to this cohort to determine their sensitivity for selecting patients with PVs in high risk ( $>4 \mathrm{x}$ risk compared to general population) breast/ovarian cancer genes. These populations were chosen because of similar healthcare systems and ancestral distribution. Results: Over $75 \%$ of patients in each country/province analyzed were OOC. Rates of PVs were similar between IC and OOC patients across countries. Existing Canadian, Australian, and UK criteria missed up to $30 \%$ of patients with high-risk PVs. The majority $(>80 \%)$ of PVs in OOC patients were in genes with published management guidelines. Conclusions: In our cohort, select international testing criteria identified $<30 \%$ of patients with PVs. These data suggest expanding certain international guidelines would allow better identification and improved management for $\mathrm{BC}$ patients across the globe.

\section{Clinical Utility of Genetic Testing in Children with Seizure Onset After 2 Years of Age}

Elvira Zilliacus ${ }^{1}$, Khalida Liaquat ${ }^{2}$, Kimberly Gall ${ }^{2}$, Emanuela Izzo ${ }^{3}$, Akashdeep Singh ${ }^{3}$, Kirsi Alakurtti ${ }^{1}$, Eija Seppala ${ }^{1}$, Lotta Koskinen ${ }^{1}$, Juha Koskenvuo ${ }^{1}$ and Tero-Pekka Alastalo ${ }^{1}$

${ }^{1}$ Blueprint Genetics, Espoo, Finland, ${ }^{2}$ Blueprint Genetics, Seattle, WA, USA and ${ }^{3}$ Biomarin Pharmaceutical Inc., Novato, CA, USA

Background: Seizure disorders secondary to neurologic and metabolic disorders are some of the most common genetic disorders presenting in childhood. A molecular diagnosis in these patients allows for etiologically based treatment and management; therefore, it is critical to maximize the diagnostic yield. 'The Paediatric Epilepsy? Look Beyond' is a sponsored program offering genetic testing to patients with epilepsy in Europe and the Middle East. Aim: To determine the overall molecular diagnostic yield in pediatric epilepsy patients between 2 and 5 years of age and assess the impact on diagnosing neuronal ceroid-lipofuscinosis type 2 (CLN2) given the opportunity to treat these patients with enzyme replacement therapy. Methods: 541 patients were tested using a comprehensive next-generation sequencing (NGS)-based epilepsy panel. Patients were age 24-48 months at the time of testing, had the first seizure at 24 months or over, and had at least one additional finding (EEG, MRI abnormalities, speech delay, or motor symptoms). Results: A genetic diagnosis was established in 115 patients (21.3\%). CNVs accounted for $16 \%$ of diagnosed patients, with $28 \%$ being intragenic. CLN2 cases received a molecular diagnosis at an average age of 3 years 11 months, 1-2 years earlier than natural history data. At least $72(62.6 \%)$ patients who received a molecular diagnosis had a disorder that has a targeted treatment, evidence for optimizing pharmaceutical treatment, or on-going clinical trials available. Conclusion: This genetic testing program demonstrates the clinical utility of a comprehensive epilepsy gene panel with high-level CNV detection for patients whose first seizure occurs at or after 2 years of age.

\section{Utility and Diagnostic Yield of Whole Exome Sequencing in Adult Patients with Intellectual Disability}

Jessica Taylor, Kirsty West, Adelaide Hopkins, Bryony Thompson, Paul James, Michael Fahey, Christine Wools, Alison Trainer, Ingrid Winship, Joshua Shultz, Adrienne Sexton, Rebecca Purvis, Elizabeth Donaldson, Emma Wright and Maie Walsh Genomic Medicine, Royal Melbourne Hospital, Melbourne, VIC, Australia

Background: Previous genetic testing for intellectual disability (ID) was often limited to conventional or molecular karyotype and testing for fragile X. Recent accessibility to whole exome sequencing (WES) has facilitated the re-evaluation of monogenic diagnoses for patients with ID who are now adults. A vast amount of literature examining WES in pediatric populations exists, however there is a paucity of literature regarding adult patients with ID. Aim: To understand diagnostic yield of WES in adults with ID and clinical and personal utility of testing outcomes. Methods: A clinical audit of adult patients who presented to our service between 2018 and 2020, and received WES testing for ID. Each case was reviewed to assess the outcome of genetic testing as well as the clinical and personal utility of results. Results: Eight (29\%) received a molecular diagnosis that matched their phenotype, 9 (32\%) had a variant of unknown significance (one with high clinical suspicion) and in the remaining 11 (39\%) cases, a molecular diagnosis was not identified. Incidental carrier status of recessive conditions was identified in several individuals. Conclusions: The clinical utility of a genetic diagnosis for adults with ID is often thought to be limited, although important for siblings. Personal utility for patients and their carer(s), following a long diagnostic odyssey is often important and 
beneficial. We present cases of interest including one case in which a genetic diagnosis resulted in change to medical management, and a second case in which a genetic diagnosis did not change medical management however facilitated efficacy of social support.

\section{Mitochondrial DNA Analysis Increases Diagnostic Yield in Patients with Inherited Retinal Disease}

Elvira Zilliacus, Pernilla von Nandelstadh, Sari Tuupanen, Marta Gandia,

Sanna Vattulainen-Collanus, Kati Kämpjärvi, Johanna Känsäkoski, Katja Merkkiniemi, Laura Sarantaus, Raquel Perez-Carro, Jonna Tallila, Ville Kytölä, Mikko Muona, Johanna Sistonen, Inka Saarinen, Juha Koskenvuo and Tero-Pekka Alastalo

Blueprint Genetics, Helsinki, Finland

Background: The mitochondrial genome ( $\mathrm{mtDNA}$ ) is a 37 -gene, circular, $16.5 \mathrm{~kb}$ genome. Mitochondrial dysfunction caused by pathogenic/likely pathogenic variants in the mtDNA has been associated with retinal disease and vision loss. Aim: To assess mtDNA variants in a cohort of patients affected by inherited retinal disease (IRD). Methods: A customized, highly sensitive, mtDNA assay was developed, based on hybridization-based capture of mtDNA and next-generation sequencing (NGS), to detect very low heteroplasmy levels of SNVs, INDELs, and deletions. The mtDNA assay was validated and included in the diagnostic sequencing of 2597 IRD patients. Results: The mean read depth for mtDNA was $18,224 \mathrm{x}$ and $100 \%$ of base pairs were covered with a sequencing depth of $>1000 x$. Sensitivity to detect SNVs and INDELs with over 10\% heteroplasmy was 100\%. For SNVs with $5-10 \%$ and $<5 \%$ heteroplasmy, the sensitivity was $93.3 \%$ and $88.9 \%$, respectively. A pathogenic/likely pathogenic mtDNA variant was identified in 22 patients $(0.85 \%)$, including one heteroplasmic large deletion (7542bp), 5 homoplasmic, and 16 heteroplasmic SNVs. Homoplasmic variants were associated mostly with Leber hereditary optic neuropathy (LHON). Diagnostic variants were identified in eight mtDNA genes; MT-ND1, MT-ND4, MT-ND6, MTATP6, MT-TN, MT-TH, MT-TL1, and MT-TV. The most common variant was MT-TL1 M.3243A>G, identified in 11 patients with retinal disease described as macular/code dystrophy. Additionally, mtDNA VUS was reported in seven cases. Conclusion: Adding mtDNA analysis to routine genetic diagnostic testing for IRD patients can increase diagnostic yield by $>0.85 \%$. Analysis of the full $\mathrm{mtDNA}$ allows the identification of several variants associated with both syndromic and nonsyndromic IRD.

\section{METABOLIC AND DIET DISEASE MANAGEMENT}

\section{The Relationship Between ß-Ureidopropionase Deficiency Due to UPB1 Variants and Human Phenotypes is Uncertain}

Sarah Righetti ${ }^{1}$, Richard Allcock ${ }^{2}$, Joy Lee ${ }^{3}$, Louisa Adams ${ }^{4}$, Carolyn Ellaway ${ }^{4}$, Kristi Jones ${ }^{4}$, Arthavan Selvanathan ${ }^{4}$, Janice Fletcher ${ }^{5}$, James Pitt ${ }^{6}$, André B P van Kuilenburg ${ }^{7}$, Martin Delatycki ${ }^{6}$, Nigel Laing ${ }^{8}$ and Edwin P Kirk

${ }^{1}$ University of New South Wales, Sydney, NSW, Australia, ${ }^{2}$ PathWest Laboratory Medicine, Perth, WA, Australia, ${ }^{3}$ Royal Children's Hospital, Melbourne, VIC, Australia, ${ }^{4}$ Sydney Children's Hospital Network, Sydney, NSW, Australia, ${ }^{5} \mathrm{New}$ South Wales Health Pathology, Sydney, NSW, Australia, ${ }^{6}$ Victorian Clinical Genetics Service, Melbourne, VIC, Australia, ${ }^{7}$ Amsterdam University Medical Center, Amsterdam, Netherlands and ${ }^{8}$ Harry Perkins Institute of Medical Research, Perth, WA, Australia

Background: $ß$-ureidopropionase deficiency, caused by variants in UPB1, has been reported in association with varied neurodevelopmental phenotypes including intellectual disability, seizures, and autism. Aim: We aimed to reassess the relationship between variants in UPB1 and a clinical phenotype, in the light of available evidence including population databases. Method: Literature review, calculation of carrier frequencies from population databases, long-term follow-up of previously published case and report of additional cases. Results: Several of the variants previously reported as pathogenic are present at higher than expected frequencies for a rare condition. In particular, the most frequently reported variant, p.Arg326Gln, is very common among East Asians, with a carrier frequency of 1 in 20 and 1 in 907 being homozygous for the variant. Moreover, published reports include multiple individuals with mild or no phenotypes, as well as nonneurological presentations. Conclusion: Pending the availability of further evidence, UPB1 should be considered a 'gene of uncertain clinical significance'. Caution should be used in ascribing diagnostic significance to the identification of biochemical features of $ß$ ureidopropionase deficiency or UPB1 variants in patients with neurodevelopmental phenotypes. UPB1 is not currently suitable for inclusion in gene panels for reproductive genetic carrier screening.

\section{MOLECULAR GENETICS}

\section{Increased Evidence Implicates Notch3Cysteine-Sparing Variants as a Cause of CADASIL}

Neven Maksemous, Paul Dunn, Robert A. Smith, Heidi G. Sutherland, Rod A. Lea and Lyn R Griffiths

Queensland University of Technology (QUT), Centre for Genomics and Personalised Health, Genomics Research Centre, School of Biomedical Sciences, Brisbane, QLD, Australia

Introduction: Cerebral autosomal dominant arteriopathy with subcortical infarcts and leukoencephalopathy (CADASIL) is the leading heritable cause of stroke and vascular dementia in adults. CADASIL is a monogenic small vessel disease (SVD) caused by missense mutations in the NOTCH3 gene. Typical CADASIL-causing mutations result in odd number of cysteine residue within the EGF-like repeats. Recurrent strokes, migraine or migraine-like headaches, progressive dementia, and pseudobulbar paralysis are the common clinical presentations of CADASIL. Atypical CADASIL variants (Cysteine-sparing) have been described in some CADASIL cases. Aim: A comprehensive genetic analysis of the cysteine sparing $\mathrm{NOTCH} 3$ variants in patients with clinical suspicion of CADASIL. Method: Genetic analysis of NOTCH3 gene, using the targeted next-generation sequencing gene panel, was performed in a large cohort $(n=$ 674) referred for molecular CADASIL testing from Australia and New Zealand. Results: One nonsense and 41 different cysteine-sparing missense variants in 115 cases (17\%) have been identified. Of these variants, 10 have not previously been reported in any available databases. Twenty-one missense variants predicted to be disease causing by at least 3 of 4 in silico prediction tools used in this study. In addition, $32 / 115(28 \%)$ cases have $\geq 2$ compound heterozygous variants in $\mathrm{NOTCH}$. Conclusion: Our findings support the view that cysteine-sparing variants might cause CADASIL with a phenotype overlapping with/similar to that from typical cysteine altering mutations. 


\section{The Clingen Kidney Disease Clinical Domain Working Group: Defining Solutions to the Challenge of Nephropathy Ontology and Nomenclature}

Alicia B. Byrne ${ }^{1,2}$, Tam P. Sneddon ${ }^{3}$, Hannah Dziadzio ${ }^{1}$, Matthew G. Sampson ${ }^{4,5,6}$, Andrew J. Mallett ${ }^{7,8,9}$ and on behalf of the ClinGen Kidney Disease Clinical Domain Working Group

${ }^{1}$ Clinical Genome Resource (ClinGen), Broad Institute of MIT and Harvard, Boston, MA, USA, ${ }^{2}$ Centre for Population Genomics, Garvan Institute of Medical Research, Sydney, NSW, Australia, ${ }^{3}$ Department of Pathology and Laboratory Medicine, University of North Carolina, Chapel Hill, NC, USA, ${ }^{4}$ Division of Nephrology, Boston Children's Hospital, Boston, MA, USA, ${ }^{5}$ Medical and Population Genetics, Broad Institute of MIT and Harvard, Cambridge, MA, USA, ${ }^{6} \mathrm{Harvard}$ Medical School, Boston, MA, USA, ${ }^{7}$ Townsville Hospital and Health Service, Townsville, QLD, Australia, ${ }^{8}$ College of Medicine and Dentistry, James Cook University, Townsville, QLD, Australia and ${ }^{9}$ Institute for Molecular Bioscience and Faculty of Medicine, The University of Queensland, Brisbane, QLD, Australia

Kidney disease affects 1 in 10 people with $10 \%$ of affected individuals having a monogenic form. The ClinGen Kidney Disease Clinical Domain Working Group (CDWG) was established in 2019 with the goal of creating a comprehensive, standardized knowledge base defining the clinical validity of gene-disease relationships and expert classifications of variant pathogenicity across the full spectrum of nephropathies. The most significant challenge however, to curating the clinical genome associated with kidney disease lies in defining the disease entity. For many nephropathies, current disease nomenclature (e.g., OMIM) and ontology (e.g., MonDO) systems do not accurately reflect the underlying biology of a given disorder and are not aligned with contemporary knowledge of disease pathogenesis. Inaccuracies in disease naming have been found to mainly arise from multiple, similar disorders being considered separate disease entities rather than a phenotypic spectrum of a single disorder, and can also result from historical misidentifications and naming based on phenotypic rather than etiologic factors. The ClinGen Kidney Disease CDWG, in coordination with the ClinGen Disease Naming Working Group, are currently defining consensus recommendations for kidney disease naming, with input from representatives across the global renal community, including clinicians, scientists, and patients. Naming will follow the general, stepwise system of 'core disease name, distinguishing subtype, gene name' allowing delineation of disease entities in an informative and biologically valid manner. Implementing these changes across the multiple related, but not interdependent, ontology, and nomenclature systems will likely be a challenging undertaking, but one that will have immense benefits in clinical practice.

\section{A $127 \mathrm{~kb}$ Truncating Deletion of PGRMC1 is a Novel Cause of X-Linked Isolated Pediatric Cataracts}

Johanna L. Jones ${ }^{1}$, Mark A. Corbett ${ }^{2}$, Elise Yeaman ${ }^{1}$, Duran Zhao ${ }^{1}$, Jozef Gecz ${ }^{2}$, Robert J. Gasperini ${ }^{3}$, Jac C. Charlesworth ${ }^{1}$, David A. Mackey ${ }^{4}$, James E. Elder ${ }^{5}$, Jamie E. Craig ${ }^{6}$ and Kathryn P. Burdon ${ }^{1,6}$

${ }^{1}$ Menzies Institute for Medical Research, University of Tasmania, Hobart, TAS, Australia, ${ }^{2}$ Robinson Research Institute, University of Adelaide, Adelaide, SA, Australia, ${ }^{3}$ School of Medicine, University of Tasmania, Hobart, TAS, Australia, ${ }^{4}$ Lions Eye Institute, University of Western Australia, Perth, WA, Australia, ${ }^{5}$ Department of Paediatrics, University of Melbourne, Melbourne, VIC, Australia and ${ }^{6}$ Department of Ophthalmology, Flinders University, Adelaide, SA, Australia

Background: Pediatric cataracts (PC) are a rare heterogenous disease causing visual impairment in children. Aim: To identify the disease-causing variant in a family with apparent X-linked PC. Methods: Parametric linkage analysis, using Illumina OmniExpress SNP array data, was performed using Merlin. Complete Genomics ${ }^{\text {ma }}$ whole-genome sequence was used to identify variants within the identified linkage regions. Rare, segregating variants were further investigated in vivo. Results: The phenotype showed linkage to a $6.8 \mathrm{Mb}$ region at Xq24 $(\mathrm{LOD}=2.53)$ and two additional regions at $1 \mathrm{q} 42.2-1 \mathrm{q} 43$ and $3 \mathrm{q} 26.31-3 \mathrm{q} 26.32(\mathrm{LOD}=2.44)$. A $127 \mathrm{~kb}$ deletion at Xq24 was identified, which truncates the PGRMC1 gene following exon 1. An ERO1B c.662C >T p.(Ala221Val) variant on chrl segregates, but was not determined to be disease-causing with an unlikely pattern of inheritance and poor in silico predictions. Additional reports were identified of two unrelated males with PGRMC1-disrupting deletions and nonsyndromic intellectual disability with congenital cataracts. Knockdown of $P G R M C 1$ in zebrafish (D. rerio) lead to cataract in early development $(p \leq .001$ compared to control morpholino), with the use of this translation blocking morpholino antisense oligo replicating full gene loss. PGRMC1 loss is hypothesized to be causing cataract through disrupted cholesterol biosynthesis. Replication and mechanistic investigation with CRISPR knockout fish are currently underway. Conclusion: A truncating deletion of PGRMC1 is the cause of the X-linked PC in this family and is a novel cause of cataracts. The identification of novel genes will expand clinical gene panels and improve diagnostic rates for this disease.

\section{A Novel ATP1A2 Mutation in a Patient with FHM Type2}

Zizi Molaee, Neven Maksemous, Robert Smith and Lyn Griffiths

Queensland University of Technology (QUT), Centre for Genomics and Personalised Health, Genomics Research Centre, School of Biomedical Sciences, Brisbane, QLD, Australia.

Background: Familial hemiplegic migraine (FHM) is a severe subtype of migraine with aura with an autosomal dominant pattern of inheritance. Mutations in the ATP1A2, CACNa2A, SCN1A genes have been implicated in the FHM patients, causing FHM1, FHM2, and FHM, respectively. The ATP1A2 gene plays a crucial role in maintaining the electrochemical gradients of sodium and potassium ions across the plasma membrane. Aim: We report a novel ATP1A2 mutation in a patient with the phenotypes resembling that of previously reported cases of FHM type 2. A 30-year-old Caucasian female presented with episodes of typical hemiplegic migraine since the age of 10. Symptoms of attacks included right hand side weakness and numbness spreading from toes to the top of the head, dysphasia, photophobia, and mild phonophobia. She also developed severe throbbing headache (1-2 hours), nausea, and vomiting. Coma was also reported which lasted from 4 hours to 3 days. Methods: All the three known FHM genes were screened using the Targeted Next Generation sequencing panel (TGP). Results: A novel heterozygous missense variant was identified in the ATP1A2 gene in exon 15, NM_000702.3, c.2066G >T, p.Arg689Leu, and confirmed via Sanger method. The p. Arg689Leu was classified as likely pathogenic according to the American College of Medical Genetics (ACMG) guidelines. Conclusion: The novel mutation (p.Arg689Leu) expands the spectrum of mutations related to FHM2 and supports the high variability in clinical manifestation within the same family.

\section{Using Long-Read Sequencing to Link DNA Methylation with a Large Repeat Region In cis in ATR-X syndrome}

Olivia Burling, Binoy Appukuttan and Karen Lower

Flinders University, Adelaide, SA, Australia

Background: ATR-X syndrome is a rare X-linked inherited disorder caused by a mutated $A T R X$ gene and is characterized by intellectual 
disability, facial abnormalities, and alpha thalassemia. Mutations in ATRX cause global changes in methylation patterns at $\mathrm{CpG}$ islands throughout the genome, including the ATRX target NME4. Understanding this phenomenon, using NME4 as a model locus, may provide valuable information about methylation establishment during development and identify potential therapeutic targets for ATR-X syndrome. Aim: Develop a novel workflow to interrogate the methylation pattern of NME4 using long-read sequencing. Method: The novel workflow will combine four methods. ATR-X patient DNA will be deaminated to allow 5-methylcytosines to be detected. The target region of NME4 will be enriched using CRISPR/Cas9 targeted enrichment. The enriched regions will be sequenced using PacBio SMRT sequencing (circular consensus sequencing). The methylation pattern will be determined from sequencing data. Results: Preliminary results have been focussed on optimising the deamination and enrichment methodologies on control and patient DNA. The optimal conditions and ratio of magnetic beads for DNA retrieval were found and consistently achieved $\sim 70 \%$ retrieval of input DNA in the deamination process. The primers sites for the CRISPR/Cas9 enrichment are currently being optimized. Conclusion: Early results are promising that the workflow can determine the methylation pattern at NME4. This novel method will create a tool to identify methylation patterns in regions of the genome that are inaccessible with traditional methods.

\section{MitoReport: Accessible Interpretation of Mitochondrial Variants}

Simon Sadedin ${ }^{1,2,3}$, Tommy $\mathrm{Li}^{1}$, David $\mathrm{Ma}^{1}$ and Katrina Bell ${ }^{1}$

${ }^{1}$ Murdoch Children's Research Institute, Melbourne, VIC, Australia,, ${ }^{2}$ Victorian Clinical Genetics Services, Melbourne, VIC, Australia and ${ }^{3}$ The University of Melbourne, Melbourne, VIC, Australia

Background: Testing for defects in mitochondrial DNA (mtDNA) is complicated by many factors ranging from technical to biological, making it significantly more challenging than identifying similarly pathogenic variants in nuclear DNA. In the past, this has inhibited many researchers from examining mitochondrial variants. Recently, however, available methods and resources have improved in three important respects, making mtDNA analysis significantly more tractable than previously. Results: We present an overview of three new important resources that significantly increase the accessibility of interpretation of mitochondrial variants. These include: the release of mitochondrial variant data in gnom $A D$, the publication of ACMG guidelines for interpretation of mitochondrial variants, and the availability of new tools including a standard mtDNA analysis pipeline from the Broad Institute, and our own variant curation tool we have developed called MitoReport. MitoReport integrates essential public data including population frequency, heteroplasmy levels, disease association, and specific haplogroup information into an easy to use interface, and accompanies it with predefined filters to aid in highlighting significant variants. Conclusion: New resources and tools significantly enhance the accessibility of mitochondrial variant interpretation, and may offer a valuable pathway to resolving causative variants in previously unsolved patients.

\section{A Novel Variant in Surfactant Protein A2 is the Likely Cause of Idiopathic Pulmonary Fibrosis in an Australian Family}

Sionne E. M. Lucas ${ }^{1}$, Kelsie Raspin ${ }^{1}$, Ian Glaspole ${ }^{2}$, Haydn Walters ${ }^{1}$, David Schwartz ${ }^{3}$, Richard Wood Baker ${ }^{4}$, Daniel Chambers ${ }^{5}$, Yuben Moodley ${ }^{6}$, Paul Reynolds ${ }^{7}$, Lauren Troy ${ }^{8}$, Simon Walsh ${ }^{9}$, Tamera Corte ${ }^{8}$ and Joanne L. Dickinson ${ }^{1}$

${ }^{1}$ Menzies Institute for Medical Research, University of Tasmania, Hobart, TAS, Australia, ${ }^{2}$ Alfred Hospital, Melbourne, VIC, Australia, ${ }^{3}$ University of Colorado, Denver, CO, US, ${ }^{4}$ Calvary Hospital, Hobart, TAS, Australia, ${ }^{5}$ The Prince Charles Hospital, Brisbane, QLD, Australia, ${ }^{6}$ Fiona Stanley Hospital, Perth, WA,

Australia, ${ }^{7}$ Royal Adelaide Hospital, Adelaide, Australia,, ${ }^{8}$ Royal Prince Alfred Hospital, Sydney, NSW, Australia and ${ }^{9}$ Kings College Hospital, London, UK

Background: Idiopathic pulmonary fibrosis (IPF) is a rare and devastating disease characterized by progressive lung scaring and functional decline, with a median survival of 2.3-6.7 years. Genetic studies demonstrate that a genetic predisposition is important in familial and sporadic IPF, though many families do not yet have a molecular diagnosis. To support further research, Australian IPF families have been recruited to our 'Genetic Research in IPF (GRIPF)' study, using the Australian IPF Registry and our network of respiratory physicians. Aim: To identify putative disease-causing variants in Australian IPF families. Methods: Genome sequencing data were generated and interrogated to identify variants that segregate with disease, are rare, and are predicted to be deleterious using in silico tools. Results: Preliminary analyses have identified a novel, putative disease-causing variant in a known IPF-gene (SFTPA2) in one of the families. The variant, c.160C $>\mathrm{T}$ (p.P54S), is shared by two affected brothers but absent in their unaffected mother, has a population frequency of 1/251,054 (gnomAD), and a CADD score of 23.3. The wild-type amino acid, proline, is located in the collagen-like domain of SFTPA2, is highly conserved, and predicted to be post-translationally modified to stabilize the protein structure. Functional assays to determine the impact of this variant are in progress. Conclusion: We have identified a novel, putative diseasecausing variant in SFTPA2 in an Australian IPF family. This is important for the family themselves, as variants in SFTPA2 are linked to both IPF and lung cancer, and broadens our understanding of the molecular basis of IPF.

\section{AMPLIDEX ${ }^{\circledR}$ SMN1 PCR: A SCALABLE SOLUTION FOR SMA TESTING}

Bianca Rodrigues, Natalia Smietanka, Corrina Cliffe and Edwin Kirk

NSW Health Pathology Genetics Randwick, Sydney, NSW, Australia

Background: Current Australasian guidelines recommend that women who are planning or in the early stages of pregnancy are offered genetic carrier screening for Fragile X syndrome, cystic fibrosis, and spinal muscular atrophy (SMA). This has led to increased demand for SMN1 exon 7 copy number testing. Aim: Currently, the NSW Health Pathology (NSWHP) Genomics Laboratory at Randwick provides SMA carrier screening using Multiplex Ligation-Dependent Probe Amplification (MLPA) for SMN1 copy number determination. This assay is costly, time-consuming, and labor intensive. By comparison, the AmplideX ${ }^{\circledast}$ PCR/CE SMN1 Kit method is cost effective and can be automated to reduce the amount of time scientific staff spends performing benchwork. Method: The AmplideX ${ }^{\circledast}$ PCR/CE SMN1 Kit is a PCR-based assay 
that relies on relative quantitation of SMN1 exon 7 compared with an endogenous control product using capillary electrophoresis and custom AmplideX ${ }^{\circledast}$ PCR/CE Reporter software for copy number determination. The AmplideX ${ }^{\circledast}$ PCR/CE SMN1 Kit method was compared to MLPA using 79 samples with known SMN1 copy number. Results: Verification of the AmplideX ${ }^{\circledR}$ SMN1 PCR kit in our laboratory demonstrated $100 \%$ sensitivity and specificity at the clinically significant thresholds of SMN1 copy numbers 0,1 , and 2 . Conclusion: Implementation of the AmplideX ${ }^{\circledR}$ SMN1 PCR kit will allow for the upscaling of carrier screening for SMA to meet increasing demand.

\section{Menstrual Migraine and its Association with HFE SNPs (rs1799945 and rs1800562)}

Thais Zielke, Heidi Sutherland and Lyn Griffiths

Queensland University of Technology, Brisbane, QLD, Australia

Background: There is evidence suggesting iron homeostasis involvement in the pathophysiology of migraine. Studies have shown correlations between duration of headache and iron deposition. In addition, high migraine prevalence was recorded in women with hereditary hemochromatosis $(\mathrm{HH})$. Homeostatic iron regulator (HFE) is the main genetic cause of $\mathrm{HH}$ and encodes the HFE protein, which is responsible for hepcidin activation. The two main polymorphisms known to cause $\mathrm{HH}$ are rs1799945 (H63D) and rs1800562 (C282Y). We hypothesized that due to HFE polymorphisms causing iron overload, it could also lead to oxidative stress in the brain, therefore generating inflammatory reactions that could result in menstrual migraine (MM). Aim: To identify whether HFE SNPs (rs1799945 and rs1800562) are associated with MM. Methods: Using a MM cohort consisting of 268 menstrual migraine cases and 142 unaffected female control, rs1799945 and rs1800562 were genotyped using Polymerase Chain Reaction - Restriction Fragment Length Polymorphism (PCR-RFLP) assays and chi-square analysis to test for significant differences between cases and controls. Results: We found a significant difference between the cases and controls for the rs1799945 ( $\mathrm{p}=0.01$ ), with the Minor Allele Frequency (MAF) higher in MM (19.5\%) compared to controls (13\%). The MAF in gnomAD is $14 \%$ for Non-Finnish Europeans, similar to our controls (13\%).Conclusion: We find that HFE rs1799945 is significantly associated with MM, suggesting that H63D may contribute to an increased susceptibility to MM.

\section{NEUROGENETICS}

\section{Research Themes in KAT6A and KAT6B Syndromes: A Scoping Review}

Tanya Tripathi ${ }^{1}$, Miya St John ${ }^{1}$, Meg Salisbury ${ }^{2}$ and David J. Amor ${ }^{1,3,4}$

${ }^{1}$ Murdoch Children's Research Institute, Melbourne, VIC, Australia, ${ }^{2}$ KAT6A Foundation, Melbourne, VIC, Australia, ${ }^{3}$ University of Melbourne, VIC, Australia and ${ }^{4}$ Royal Children's Hospital, Melbourne, VIC, Australia

Background: Pathogenic variants in KAT6A and KAT6B genes cause syndromic neurodevelopmental disorders. KAT6A/KAT6B syndromes are extremely rare genetic disorders with a wide variety of signs and symptoms that vary according to the location of gene variants. Common features include intellectual disability, motor delay, speech and language deficits, feeding difficulties, and skeletal abnormalities. As of 2020, 300 individuals have been diagnosed with KAT6A syndrome and $\sim 100$ with KAT6B syndrome. Aim: A comprehensive up-to-date synthesis of the research status would aid the identification of existing research themes and assist support groups such as the KAT6A Foundation to achieve their mission. Our objective was to review the published evidence regarding KAT6A and KAT6B and create a comprehensive report. Methods: Five databases (Ovid MEDLINE, Ovid EMBASE, PubMed, Web of Science, and Scopus) were systematically searched up to $29^{\text {th }}$ March 2021. To extract relevant literature, the following subject headings and keywords were combined using appropriate Boolean operators: KAT6A, KAT6B, Genitopatellar syndrome, Say-Barber-Biesecker-YoungSimpson syndrome, Lysine acetyltransferase, Histone acetyltransferase, KAT, MOZ, and MYST3. Our review included original, peerreviewed articles, and conference abstracts. The selection process comprised two stages: an initial stage involving title and abstract review and second stage involving full-text review. Results: Our search strategy yielded 638 citations that were screened further by title and abstract. Of these, 410 articles were excluded, leaving 228 full-text articles for more detailed review. Our synthesis of these articles provides a thorough overview of current knowledge and the status of research regarding KAT6A/KAT6B syndromes.

\section{Vitamin D Receptor Mutations Influence on Course of Parkinson's Disease in Patients Treated With Levodopa}

Jan Koper ${ }^{1}$, Barbara Zapała ${ }^{1}$, Agnieszka Spychałowicz ${ }^{2}$, Monika Piwowar ${ }^{3}$, Urszula Ciałowicz ${ }^{1}$, Adrianna Wąsińska ${ }^{1}$ and Olaf Chmura $^{1}$

${ }^{1}$ Department of Clinical Biochemistry, Jagiellonian University Medical College, Cracow, Poland, '2Department of Rehabilitation, The Center of Movement Organ Rehabilitation 'Krzeszowice' SP ZOZ, Cracow, Poland and ${ }^{3}$ Department of Bioinformatics and Telemedicine, Jagiellonian University Medical College, Cracow, Poland

Background: Parkinson's disease is the second most often occuring neurodegenerative disease after Alzheimer's disease. Vitamin Dis steroid hormone crucial for calcium homeostasis and bone metabolism. Several animal studies showed potential protective attributes of VD in dopamine cells. Aim: The aim of the study was to search for the connections between VDR gene mutations and course of PD development. Methods: Sequential analysis of VDR gene was performed on genomic DNA isolated from peripheral blood leukocytes of 100 patients with diagnosed Parkinson's Disease treated with Levodopa. Sanger sequencing was performed, we have also performed tandem mass spectrometry (LC-MS/MS) of Vitamin D metabolites. Results: From analyzed VDR gene fragments splicing region of exon 1 turned out to be the most interesting one. Mutation of 'start' (ATG) codon was detected in most cases. In examined patients, C/C genotype was present 32 times, C/T 53 times, and T/T 23 times. Patients in research group had a statistically significant prevalence of SNP. We found that dominant $\mathrm{C} / \mathrm{C}$ alleles showed statistically earlier average age of diagnosis. In addition, the presence of each subsequent $\mathrm{T}$ allele significantly delayed the onset of the disease $(p=.014)$. We have also connected $\mathrm{C} / \mathrm{T}$ genotype of rs2228570 variant with a higher chance of levodopa-induced dyskinesias. Detected metabolites concentrations also further underline this connection. Conclusion: We conclude that VDR gene mutations may influence the course of Parkinson's disease. Widely advised Vitamin D supplementation may not have an expected impact on the course of Patients with such changes. 


\section{An Integrated Neurogenomics Clinic - 28 Months Experience and Outcome of a Tertiary Referral Centre}

Alison McLean ${ }^{4,8}$, Michel Tchan ${ }^{4,9}$, Sophie Devery ${ }^{4}$, Renee Smyth ${ }^{4}$, Kishore Kumar ${ }^{4,6,7}$, Susan Tomlinson ${ }^{2,3,5}$, Stephen Tisch ${ }^{1,2,5}$ and Kathy H. C. Wu $\mathbf{W}^{1,2,3,4}$

${ }^{1}$ University of New South Wales, Sydney, NSW, Australia, ${ }^{2}$ University of Notre Dame Sydney, NSW, Australia, ${ }^{3}$ University of Sydney; , ${ }^{4}$ Clinical Genomics Unit, St Vincent's Hospital, Sydney, NSW, Australia, ${ }^{5}$ Department of Neurology, St Vincent's Hospital, Sydney, NSW, Australia, ${ }^{6}$ Molecular Medicine in Neurology, Concord Repatriation General Hospital; , ${ }^{7}$ Garvan Institute of Medical Research Sydney, NSW, Australia, ${ }^{8}$ University of New South Wales, Sydney, NSW, Australia and ${ }^{9}$ Department of Genetic Medicine, Westmead Hospital, Sydney, NSW, Australia; *These authors contributed equally

Aim: To retrospectively review attendance and outcomes of a single center, integrated multi-disciplinary neurogenomics clinic at $\mathrm{St}$ Vincent's Hospital, Sydney, Australia. Methods: An audit of patients who attended the neurogenomics clinic was conducted over a 28month period from 2017 to 2020 . The clinic comprises neurologists, clinical geneticists, and genetic counselors assessing each of the patients concurrently during the consultation. Results: In the audit period, 99 new patients were referred spanning 45 different clinical diagnoses. Following clinical assessment, $23 \%(n=23)$ of referring diagnoses were revised. 79 patients $(80 \%)$ undergoing genetic testing. The types of genetic testing ordered include 41 exome-based panels, 14 whole genome sequencing, 13 single gene tests, 30 repeat disorders, and 2 chromosomal microarrays. A diagnosis was achieved in 29 patients (29\%), of which the majority $(n=22,79 \%)$ were confirmed with molecular testing. The remainder had a clinical diagnosis where genetic testing was not undertaken. Of 79 patients who had genetic testing, 57 patients (58\%) did not get a molecular confirmation of the clinical diagnosis. Following testing, $2 \%$ of patients $(n=2)$ had their diagnosis revised. From referral to the results of genetic testing 25\% $(n=25)$ patients had their diagnosis revised. Conclusions: Provision of an integrated multidisciplinary neurogenomics clinic in a tertiary setting provides an invaluable service with a diagnostic yield of $30 \%$. Such a model is a gold standard for diagnostic evaluation of patients with suspected neurogenetic diagnosis. Psychosocial benefits for patients, such as convenience/satisfaction of an MDT clinic, psychological closure for patients/families, and reproductive options enabled by achieving a genetic diagnosis, will be audited via a patient survey.

\section{Deciphering the Role of Altered THOC2/TREX mRNA Export in Neurodevelopmental Disorders Using a Patient-Inspired Thoc2 Mouse Model}

Rudrarup Bhattacharjee ${ }^{1}$, Melissa White ${ }^{2}$, Paul Thomas ${ }^{2}$, Lachlan Jolly ${ }^{3}$, Jozef Gecz ${ }^{3}$ and Raman Kumar ${ }^{3}$

${ }^{1}$ Adelaide Medical School, The University of Adelaide, Adelaide, SA, Australia, ${ }^{2}$ South Australian Genome Editing Facility, SAHMRI and The Robinson Research Institute, The University of Adelaide, Adelaide, SA, Australia and ${ }^{3}$ Adelaide Medical School and The Robinson Research Institute, The University of Adelaide, Adelaide, SA, Australia

Background: We have implicated TREX-mediated mRNA export from the cell nucleus to the cytoplasm in neurodevelopmental disorders (NDDs) through the identification of pathogenic variants in the X-linked THOC2 gene. We have identified and studied $>25$ variants in patients with variable neurocognitive phenotypes. The mechanism of how altered THOC2/TREX function causes NDDs is not well understood. Aim: To generate a Thoc2 Exon 37-38 deletion mouse model, similar to a patient variant, to study the molecular, cellular, and behavioral consequences of compromised THOC2/TREX function. Methods: Extensive investigations on the Thoc2 Ex37-38 del mouse model generated by CRISPR/
Cas genome editing, after several attempts to introduce SNVs failed. Results: Thoc2 Ex 37-38 del is the first mouse model of the TREX complex. We have: (1) Confirmed absence of off-target effects due to CRISPR/Cas editing by WGS. (2) Thoc2 Ex37-38 del male mice are smaller in size and weight ( $\square 15 \%)$ compared to their wild-type littermates; a phenotype similar to the patient. (3) Thoc2 protein in Ex3738 del male mice cortices is smaller than the wild-type protein. 4. Ex37-38 sequences are deleted in male Thoc 2 mRNAs. We are currently undertaking behavioral, in vivo and in vitro neuronal assays along with transcriptomic and proteomic investigations to determine consequences of Ex37-38 del on neural cell development, differentiation, and function. Conclusion: We have generated a mouse model of THOC2/TREX complex taking advantage of a specific patient variant and phenotype, which we show is genuinely recapitulated in this mouse model paving way for detailed cellular and molecular investigations.

\section{Review of Literature on Genes Reported with Spinal Muscular Atrophy}

Prashant K Verma, Radhapyari Lourembam, Vinod Kumar and Swathi Chacham

Department of Paediatrics, All India Institute of Medical Sciences, Rishikesh, Uttarakhand, India

Background: Spinal muscular atrophy (SMA) is a heterogeneous group of disorders categorized by degeneration of anterior horn cells (AHC) in the spinal cord. SMA is the second most common autosomal recessive disorder and most commonly reported with SMN gene mutations. SMA type 1 is the most commonly reported lethal genetic cause of infant mortality. Genes reported with SMA could be classified according to clinical phenotype and molecular basis. There are various other non-SMN genes in online medical databases that lead to AHC degeneration and can be classified as non-SMN SMA. There is a paucity of literature for reviewing these genes, discussing the associated genotype and phenotype, and investigational strategy for SMN mutation-negative SMA cases. Aim: To review all SMN and non-SMN genes reported with SMA regarding clinical phenotype and investigational strategy. Materials and Methods: Single gene disorders by search with a phrase within a field (('SPINAL MUSCULAR ATROPHY')) from OMIM and PubMed are downloaded and analyzed. The authors have tried to classify all these entries on the basis of genotype and phenotype. Results: All genes can be clinically classified in four categories, but the functional classification of genes was not in order. Conclusion: Genes related to SMA are most commonly delineated with $S M N$ genes, but non-SMN genes are also not a rare cause for SMA. Initially, cases may be asymptomatic with non-SMA genes, so those cases need to be followed up. Proper diagnosis is helpful for management and genetic counseling. The molecular approach may help to define pleiotropy.

\section{Influence of Genetic Variability on the Clinical Course of Parkinsons Disease and Efficacy of Levodopa Treatment}

Olaf Chmura ${ }^{1}$, Barbara Zapała ${ }^{1}$, Agnieszka Spychałowicz ${ }^{2}$, Monika Piwowar ${ }^{3}$, Sylwia Czekalska ${ }^{1}$, Magdalena Zawada ${ }^{1}$, Adrianna Wąsińska ${ }^{1}$ and Jan Koper ${ }^{1}$

${ }^{1}$ Department of Clinical Biochemistry, Jagiellonian University Medical College, Cracow, Poland, ${ }^{2}$ Department of Rehabilitation, The Center of Movement Organ Rehabilitation 'Krzeszowice' SP ZOZ, Cracow, Poland, ${ }^{3}$ Department of Bioinformatics and Telemedicine, Jagiellonian University Medical College, Cracow, Poland and ${ }^{4}$ Department of Hematology Diagnostics, The University Hospital, Cracow, Poland

Background: Parkinson's disease is the second most common neurodegenerative disorder in the world with Levodopa being the gold 
therapeutic standard. The response to levodopa treatment may be varied, possible from the fact that the genetic variability may determine the response to the treatment. Aim: The aim of this work was to investigate the impact of genetic variants in the genes coding monoamine oxidase $\mathrm{B}$ (MAOB), dopamine receptor D2 (DRD2), and DOPA decarboxylase (DDC) on the observed differences in the clinical course of Parkinson's disease and the effects of levodopa treatment in the diagnosed patients. Methods: 126 patients: women and men aged 39-95 were included in the study. Peripheral blood samples were drawn, the genetic material in the form of DNA was extracted and the genotyping of single nucleotide polymorphisms (SNP) was performed using the TaqMan probes. Results: We detected rs2283265 and rs1076560 genetic variants of the DRD2 gene determining more frequent presence of dementia and higher patients' scores in the II and III part of the UPDRS $(p<.05)$ with results regarding the levodopa side effects did not reach statistical significance. We also demonstrate that the rs1799836 genetic variant of the MAOB gene and rs921451 of the DDC gene does not affect the clinical course of Parkinson's disease $(p>.05)$.

Conclusion: We believe that genetic sequencing may in the future serve as an tool to assess the future course of PD.

\section{Rett Syndrome and KAT6A-Related Disorders: Chromatinopathies With Overlapping Clinical Trajectories}

Simranpreet Kaur ${ }^{1,2}$, Nicole J. Van Bergen ${ }^{1,2}$, Bruria Ben-Zeev ${ }^{3,4,5}$, Emanuela Leonardi ${ }^{6,7}$, Tiong Y. Tan ${ }^{2,8}$, David Coman ${ }^{9,10,11}$, Benjamin Kamien ${ }^{12,13}$, Susan M. White ${ }^{2,8}$, Miya St John ${ }^{1,14}$, Dean Phelan ${ }^{8}$, Kristin Rigbye ${ }^{8}$, Sze Chern Lim ${ }^{8}$, Michelle C. Torres $^{8}$, Melanie Marty ${ }^{8}$, Elena Savva ${ }^{8}$, Teresa Zhao ${ }^{8}$, Sean Massey ${ }^{1}$, Alessandra Murgia ${ }^{6,7}$, Wendy A. Gold ${ }^{15,16,17}$ and John Christodoulou ${ }^{1,2,8,15}$

${ }^{1}$ Murdoch Children's Research Institute, Royal Children's Hospital, Melbourne, VIC, Australia, ${ }^{2}$ Department of Paediatrics, University of Melbourne, Melbourne, VIC, Australia, ${ }^{3}$ Paediatric Neurology Institute, The Edmond and Lily Safra Children's Hospital, Israel, ${ }^{4}$ Chaim Sheba Medical Center, Tel HaShomer, Israel, ${ }^{5}$ Sackler School of Medicine, Tel Aviv University, Tel Aviv, Israel, ${ }^{6}$ Department of Woman and Child Health, University of Padova, Italy, ${ }^{7}$ Fondazione Istituto di Ricerca Pediatrica, Città della Speranza, Padova, Italy, ${ }^{8}$ Victorian Clinical Genetics Services, Melbourne, VIC, Australia, ${ }^{9}$ Department of Paediatrics, The Wesley Hospital, Brisbane, Brisbane, QLD, Australia, ${ }^{10}$ Queensland Children's Hospital, Brisbane, QLD, Australia, ${ }^{11}$ School of Medicine, University of Queensland, Brisbane, QLD, Australia, ${ }^{12}$ Genetic Services of Western Australia, WA, Australia, ${ }^{13}$ Faculty of Health and Medical Sciences, University of Western Australia, WA, Australia, ${ }^{14}$ Department of Audiology and Speech Pathology, University of Melbourne, VIC, Australia, ${ }^{15}$ The University of Sydney, Faculty of Medicine and Health, Sydney, NSW, Australia, ${ }^{16}$ Kids Research, Westmead Children's Hospital, Westmead, Sydney, NSW, Australia and ${ }^{17}$ Kids Neuroscience Centre, Children's Hospital at Westmead, Westmead, Sydney, NSW, Australia

Background: Chromatin regulation is one of the most notable pathways affected in neurodevelopmental disorders (NDDs) including Rett syndrome (RTT), a pediatric-onset progressive neurological condition. Individuals with some but not enough diagnostic criteria are often referred to as RTT-like. Although RTT is mainly caused by pathogenic variants in the $\mathrm{X}$-linked transcriptional regulator Methyl-CpG-binding protein 2 (MECP2) gene, up to $13 \%$ of RTT individuals are genetically undiagnosed. Aim: We aimed to use the combination of next-generation sequencing (NGS) in a cohort of genetically undiagnosed RTT/RTT-like individuals to better understand the genetic and clinical spectrum of RTT and related NDDs. Methods: Using NGS, we have identified five RTT/RTT-like individuals with pathogenic variants in KAT6A. We then undertook a thorough systematic re-assessment of 76 previously published individuals with KAT6A-related disorder (Kennedy et al., 2019), which led to the re-classification of an additional two patients as RTT/RTTlike according to the Neul diagnostic criteria for RTT. Results: We report seven individuals with pathogenic variants in KAT6A who have overlapping clinical features with RTT and KAT6A-related disorders. All de novo heterozygous, late truncating variants were clustered in exon 17 of KAT6A and resulted in loss of the well-conserved C-terminal domain critical for binding of transcriptional co-regulators for regulation of target genes expression. Conclusion: Although variants in KAT6A have been previously associated with KAT6Arelated disorders, the association with RTT appears to have been previously unrecognized. This is the first report linking RTT/RTT-like clinical phenotypes with pathogenic variants in KAT6A, expanding the overlapping landscape of NDDs.

\section{Clinical and Genetic Review of 263 CTNNB1 Germline Variants in Neurodevelopmental Disorders}

Sayaka Kayumi $^{1}$, Luis A. Pérez-Jurado ${ }^{2}$, María Palomares ${ }^{3}$, Sarah E. Sheppard ${ }^{4}$, Wendy K. Chung ${ }^{5}$, Michael Kruer ${ }^{6}$, David Amor ${ }^{7}$, George McGillivray ${ }^{8}$, Clare van Eyk ${ }^{1}$, Kelly Harper ${ }^{1}$, Dani Webber ${ }^{1}$, Jesia Berry ${ }^{1}$, Lachlan Jolly ${ }^{1}$, Sixto García-Miñaúr ${ }^{3}$, Fernando Santos-Simarro ${ }^{3}$, Somayeh Bakhtiari ${ }^{6}$, Matt Deardorff ${ }^{4,9,12}$, Holly A Dubbs ${ }^{10}$ Kosuke Izumi ${ }^{4,9,13}$, Katheryn Grand ${ }^{4,11}$, Christopher Gray ${ }^{4,9}$, Xilma R. Ortiz-Gonalez ${ }^{10,13}$, Sneha Rnagu ${ }^{4}$, Beth Keena ${ }^{4}$, Elaine Zackai ${ }^{4,13}$, Guiomar Pérez de Nanclares ${ }^{14}$, Isabel Llano ${ }^{15}$, Ignacio Arroyo ${ }^{16}$, María Ángeles Fernández-Cuesta ${ }^{17}$, Christel Thauvin ${ }^{18}$, Laurence Faivre $^{18}$, Eva Brilstra ${ }^{19}$, Gaetan Lesca ${ }^{20}$, Laurence Lion-François ${ }^{21}$, Amelie Piton ${ }^{22}$, Mathilde Nizon ${ }^{23}$, Benjamin Cogne ${ }^{23}$, Siddharth Srivastava ${ }^{24}$, Jennifer Bassetti ${ }^{24}$, Candace Muss ${ }^{25}$, Karen W. Gripp ${ }^{25}$, Rebecca A. Procopio ${ }^{25}$, Francisca Millan Zamora ${ }^{26}$, Michelle Morrow ${ }^{26}$, Melissa Assaf ${ }^{27}$, Alastair MacLennan ${ }^{1}$, Jozef Gecz ${ }^{1,28}$ and Mark Corbett ${ }^{1}$

${ }^{1}$ Adelaide Medical School, The University of Adelaide, Adelaide, SA, Australia, ${ }^{2}$ Hospital del Mar Research Institute (IMIM), Network Research Centre for Rare Diseases (CIBERER) and Universitat Pompeu Fabra, Barcelona, Spain, ${ }^{3}$ Instituto de Genética Médica y Molecular (INGEMM), Hospital Universitario La Paz, Madrid, Spain, ${ }^{4}$ Division of Human Genetics, Children's Hospital of Philadelphia, Philadelphia, PA, USA, ${ }^{5}$ Columbia University, New York, NY, USA, ${ }^{6}$ Pediatric Movement Disorders Program, Division of Pediatric Neurology, Barrow Neurological Institute, Phoenix Children's Hospital, Phoenix, Arizona, USA, ${ }^{7}$ Department of Paediatrics, The University of Melbourne, Melbourne, VIC, Australia, ${ }^{8}$ Murdoch Children's Research Institute, Melbourne, VIC, Australia, ${ }^{9}$ Robert's Individualized Medical Genetics Center, Children's Hospital of Philadelphia, Philadelphia, PA, USA, ${ }^{10}$ Division of Neurology, Children's Hospital of Philadelphia, Philadelphia, PA, USA, ${ }^{11}$ Current affiliation Department of Pediatrics, Medical Genetics, Cedars-Sinai Medical Center, Los Angeles, CA, USA, ${ }^{12}$ Current affiliation Departments of Pathology and Laboratory Medicine and Pediatrics, Children's Hospital Los Angeles, Keck School of Medicine of the University of Southern California, Los Angeles, CA USA, ${ }^{13}$ Perelman School of Medicine, University of Pennsylvania, Philadelphia, PA, USA, ${ }^{14}$ Hospital Txagorritxu, Vitoria, Spain, ${ }^{15} \mathrm{Hospital}$ de Cruces, Barakaldo, Spain, ${ }^{16} \mathrm{Hospital}$ de Cáceres, Cáceres, Spain, ${ }^{17} \mathrm{Hospital}$ de Basurto, Bilbao, Spain, ${ }^{18} \mathrm{CHU}$ Dijon Bourgogne, Dijon, France, ${ }^{19} \mathrm{UMC}$ Utrecht, Utrecht, the Netherlands, ${ }^{20}$ Department of Medical Genetics, Hospices Civils de Lyon, Lyon, France, ${ }^{21}$ Department of Pediatric Neuology, Hospices Civils de Lyon, Lyon, France, ${ }^{22}$ Department of Medical Genetics, Hopitaux Universitaires de Strasbourg, France, ${ }^{23} \mathrm{CHU}$ Nantes, Nantes, France, ${ }^{24}$ Children's Harvard, MA, USA, ${ }^{25}$ Nemours/A.I duPont Hospital for Children, Wilmington, DE, USA, ${ }^{26}$ GeneDX, Gaithersburg, MD, USA, ${ }^{27}$ Banner Childrens Specialists Neurology Clinic, Glendale, AZ, USA and ${ }^{28}$ South Australian Health and Medical Research Institute, Adelaide, SA, Australia

The CTNNB1 gene encodes the $\beta$-catenin phosphoprotein that is best known for its dual roles in regulation of Wnt signaling pathway and cell-cell adhesion. Heterozygous, loss-of-function, or dominant-negative germline variants of CTNNB1 cause neurodevelopmental disorders, while somatic gain-of-function variants are enriched in cancers. We aimed to characterize the clinical spectrum of CTNNB1 syndrome in 41 novel and 222 additional individuals from 
the literature and public clinical-genetic databases. Aggregation of clinical information revealed a relatively homogeneous syndrome encompassing motor delay (90.7\%), developmental delay $(90.7 \%)$, craniofacial dysmorphism $(88.1 \%)$, delayed speech and language (87.1\%), truncal hypotonia $(84.1 \%)$, eye defects $(83.5 \%)$, microcephaly $(80.4 \%)$, peripheral spasticity/hypertonia (75.9\%), intellectual disability (74.6\%), and behavioral abnormalities (70.7\%). Using Phenomizer, the combination of these 10 phenotypes differentially diagnosed Angelman syndrome (MIM: 105830), with absence of seizures a distinguishing feature of CTNNB1 syndrome. Ninety percent $(237 / 263)$ of CTNNB1 variants we identified were loss-of-function and expression of $\beta$-catenin mRNA was reduced due to nonsense mediated mRNA decay in two available patient lymphoblastoid cell lines. To aid clinical interpretation of missense variants of uncertain significance (accounting for 20/263; $7.6 \%$ of the cohort), we implemented a functional genomic test based on the role of $\beta$-catenin in Wnt signaling (TOPFlash luciferase reporter assay). Of three missense CTNNB1 variants selected based on patient phenotypes, two were dominant negative and significantly repressed reporter gene expression. The TOPFlash luciferase reporter assay is therefore effective for resolving the pathogenicity of some missense variants for individuals fitting our defined clinical picture of CTNNB1 syndrome.

\section{Massimo's Mission: Developing Better Outcomes for Australian Patients with Leukodystrophies}

Eloise Uebergang ${ }^{1,2,3,4}$, Chloe Stutterd ${ }^{1,3,4,5}$, Chris Love ${ }^{1}$, Nicholas Smith ${ }^{6}$, Mohammed R. Shaker ${ }^{7}$, Dominik Froehlich ${ }^{8}$, Stephen Damiani ${ }^{9}$, Matthias Klugmann ${ }^{8}$, Ernst J. Wolvetang ${ }^{7}$, Cas Simons ${ }^{1}$ and Richard J Leventer ${ }^{1,2,3,5}$

${ }^{1}$ Murdoch Children's Research Institute, Melbourne, VIC, Australia, ${ }^{2}$ Australian Genomics Health Alliance, Melbourne, VIC, Australia, ${ }^{3}$ Royal Children's Hospital, Melbourne, VIC, Australia, ${ }^{4}$ Victorian Clinical Genetics Services, Melbourne, VIC, Australia, ${ }^{5}$ The University of Melbourne, VIC, Australia, 6 Womens and Children's Hospital and University of Adelaide, SA, Australia, ${ }^{5}$ The University of Melbourne, VIC, Australia, 6 Womens and Children's Hospital and University of Adelaide, SA, Australia, ${ }^{7}$ Australian Institute for Bioengineering and Nanotechnology, The University of Queensland, Brisbane, QLD, Australia, ${ }^{8}$ Translational Neuroscience Facility and Department of Physiology, University of New South Wales, NSW, Australia and ${ }^{9}$ Mission Massimo Foundation, VIC, Australia

Background: Leukodystrophies are genetic white matter disorders that are estimated to have an incidence of 1 in 7000 live births. These conditions are often associated with an early onset, lack of treatment options, severe symptoms, and premature death. Aim: Massimo's Mission builds on the Australian Genomics Leukodystrophy Flagship and aims to (1) provide increased genetic diagnoses for Australian patients with leukodystrophies and (2) pilot the rapid development of cell and animal models for pre-clinical testing of candidate-targeted therapies. Methods: Patients are recruited through clinical services, patient groups, and online advertisements. Registry data is collected and managed using REDCap. Undiagnosed patients are provided clinical trio whole genome sequencing with a turnaround time of 15 working days. Those that remain without a diagnosis are triaged into our research program. Human-induced pluripotent stem cell (hIPSC) and rodent models of leukodystrophies are being established and comprehensively characterized. These models comprise hIPSCs and transgenic mice carrying disease-causing mutations as well as conditional knockout mice. Results: We have established the first patient registry for Australians with white matter disorders $(N=131)$ and a monthly clinic at the Royal Children's Hospital in Melbourne. Analysis of unsolved cases is underway
$(N=25)$. The stem cell and mouse disease models have significantly advanced the understanding of the pathophysiology underlying leukodystrophies and have enabled therapeutic proof-of-concept studies. Conclusion: This research is important for improved diagnosis and treatment of leukodystrophies and will contribute natural history data to assess the efficacy of future clinical trials.

\section{NEWBORN SCREENING}

\section{Principles of Genomic Newborn Screening Programs: A Systematic Review}

Lilian Downie $^{1,2}$, Jane Halliday ${ }^{1,2}$, Sharon Lewis ${ }^{1,2}$ and David J. Amor ${ }^{1,2,3,4}$

${ }^{1}$ Murdoch Children's Research Institute, Melbourne, VIC, Australia, ${ }^{2}$ Department of Paediatrics, University of Melbourne, Melbourne, VIC, Australia, ${ }^{3}$ Royal Children's Hospital, Melbourne, VIC, Australia and ${ }^{4}$ Victorian Clinical Genetics Services, Melbourne, VIC, Australia

Background: Genomic newborn screening (gNBS) may optimize health and wellbeing. Screening programs are required to be evidence-based. Aim: We aimed to provide a literature review to identify what is known and provide key points for the design of gNBS. Methods: A systematic literature review identified 36 articles that addressed: What is the interest and what would be the uptake of gNBS? What diseases and genes should be included? What is the validity and utility of gNBS? What are the ethical, legal, and social implications? Articles were only included if they generated new evidence, all opinion pieces were excluded. Results: There is consistently high interest and support for expanding to gNBS from surveyed populations. Key findings were the need for equitable access, education, and flexible consent. Gene-disease inclusion is contentious and varies according to the process used to prioritise conditions. The process for gene selection should be transparent and reflect that parents value certainty of a result in predicting disease over actionability. Analysis should minimise uncertainty and incidental findings. The expansion of screening needs to be balanced against the complexity of consenting parents for genomic testing and the risk that overall uptake of screening may decline. The literature reflects that the right of a child to self-determination is valued above the possibility of the whole family benefiting from gNBS. Conclusion: Overall, the literature supports the use of gNBS. Its inevitability therefore requires that a rigorous, evidence-based and nuanced approach is taken when designing and evaluating programs.

\section{Prenatal Genomic Testing: Towards Early Pregnancy Counseling}

Lesley Rawlings ${ }^{1}$, Wendy Waters ${ }^{1}$, Keryn Simons ${ }^{1}$, Melissa Ellul ${ }^{1}$, Cassandra Vakulin ${ }^{1}$, Amanda Wells ${ }^{1}$, Song $\mathrm{Gao}^{1}$, Julien Soubrier ${ }^{1,2}$, Rosalie Kenyon ${ }^{3}$, Ming Lin ${ }^{3}$, Rob King ${ }^{3}$, David Lawrence ${ }^{3}$, Sarah L King-Smith ${ }^{1,4}$, Abhi Kulkarni ${ }^{1}$, Chris Barnett ${ }^{5}$, Lesley McGregor ${ }^{5}$, Jan Liebelt ${ }^{5}$, Tristan SE Hardy ${ }^{1,6}$, Hamish S Scott ${ }^{1,4}$, Janice Fletcher ${ }^{1,7}$ and Karin S Kassahn $n^{1,4}$

${ }^{1}$ Genetics and Molecular Pathology, Adelaide, SA, Australia, ${ }^{2}$ School of Biological Sciences, University of Adelaide, Adelaide, SA, Australia, ${ }^{3}$ ACRF Cancer Genomics Facility, SA Pathology, Adelaide, SA, Australia, ${ }^{4}$ Centre for Cancer Biology, An alliance between SA Pathology and the University of South Australia, Adelaide, SA, Australia, ${ }^{5}$ Paediatric and Reproductive Genetics Unit, Women's and Children's Hospital, Adelaide, SA, Australia, ${ }^{6}$ Repromed, Adelaide, SA, Australia and ${ }^{7}$ NSW Health Pathology, Sydney, NSW, Australia

Background: Since 2018, we have performed prenatal and postmortem fetal hydrops testing as virtual panels on an exome backbone. 
In 2020, we introduced prenatal exome trios for real-time clinical decision making. Aim: We reviewed the outcomes of testing in 2019 and 2020. Methods: An audit of the number of referrals, diagnostic outcomes, and evaluation of rapid trio versus panel testing was performed. Results: 13 trio and 51 singleton prenatal tests were reviewed. Turn-around times were significantly faster for trios than for singletons (14 days vs. 44 days), reflecting the desired change in practise for genomic results to be available as early as possible. Diagnostic rate for fetal hydrops was $28 \%$ and $33 \%$ for trio vs virtual panels, respectively, and $14 \%$ to $27 \%$ for other fetal abnormalities. Noonan syndrome was the predominant cause for prenatal hydrops ( $4 \mathrm{x} P T N 11,3 \mathrm{x}$ LZTR $1,1 \mathrm{x}$ RIT1, 1x SOS1), while Costello syndrome (HRAS), MPS-VII (GUSB), and Escobar syndrome (CHRNG) were other causes. Lymphatic genes were identified in 4 other pregnancies ( $3 \mathrm{x}$ PIEZO1, 1x FOXC2). Compound heterozygous, pathogenic $N E B$ variants were detected in a fetus with bilateral talipes, possible short limbs and raised NT. The report was issued at $21+3$ weeks gestation. In a pregnancy with fetal cardiac anomalies and AVSD no genetic cause was identified by trio exome analysis. After birth, the baby deteriorated and cardiac surgery was considered. Rapid review of the existing exome data allowed assessment for Kabuki syndrome which was also negative. Conclusion: We have successfully expanded our prenatal genomic testing to offer early trio testing to assist in real-time pregnancy counseling.

\section{Psychosocial Benefits and Harms of Noninvasive Prenatal Screening: Clinician and Patient Perspectives}

\footnotetext{
Shannon McKinn ${ }^{1}$, Nasrin Javid2'3, Ainsley Newson ${ }^{1}$, Carissa Bonner ${ }^{1}$, Natasha Nassar ${ }^{4}$, Antonia Shand4'5, Lucinda Freeman ${ }^{6}$, Sally Wortley ${ }^{1}$, Meredith Wilson ${ }^{7}$, Kirsten McCaffery ${ }^{1}$ and Katy Bell ${ }^{1}$

${ }^{1}$ The University of Sydney, Faculty of Medicine and Health, School of Public Health, Sydney, NSW, Australia, ${ }^{2}$ Royal Prince Alfred Hospital, Sydney, NSW, Australia, ${ }^{3}$ Sydney Institute for Women, Children and their Families, Sydney, NSW, Australia, ${ }^{4}$ The University of Sydney, Faculty of Medicine and Health, Children's Hospital at Westmead Clinical School, Sydney, NSW, Australia, ${ }^{5}$ Royal Hospital for Women, Department of Maternal Fetal Medicine, Sydney, NSW, Australia, ${ }^{6}$ University of New South Wales, School of Women and Children's Health, Sydney, NSW, Australia and ${ }^{7}$ Department of Clinical Genetics, Children's Hospital at Westmead, Sydney, NSW, Australia
}

Background: Noninvasive prenatal screening (NIPS) is being increasingly used by expectant parents. Much provision of this test in Australia is occurring in clinical settings where specialized genetic counseling is unavailable, such as general practice. Potential psychosocial consequences from this kind of prenatal genetic screening remain largely unexplored. Aim: This study aimed to investigate potential psychosocial consequences from prenatal genetic screening tests. Methods: We conducted a thematic analysis of semistructured qualitative interviews with 17 Australian clinicians who order, interpret, and/or discuss results of prenatal genetic tests with expectant parents, and with 13 women who received an increased-chance prenatal screening result. Results: While clinicians perceive NIPS to have important benefits, most identified unintended negative consequences arising from how NIPS was offered, and how results were delivered; potentially resulting in false reassurance or increased patient anxiety, distress, and uncertainty. Most women interviewed had positive opinions of NIPS. However, some received inadequate pretest counseling which potentially contributed to negative psychosocial consequences, including increased anxiety and distress. Several women were unaware of the genetic conditions that NIPS screened for and were subsequently surprised when they received increased chance results for these conditions. Increased-chance results, both true and false positives, caused some women to feel that bonding with their fetus had been significantly disrupted while they awaited diagnostic test results. Conclusion: Our results indicate that there is a need for clinician training and guidelines around the interpretation of prenatal genetic tests and pre- and posttest counseling, particularly as NIPS continues to be ordered in general practice.

\section{Australian Application of Novel Prenatal Rasopathy Testing Guidelines}

Gulvir Gill ${ }^{1}$, Melissa Graetz ${ }^{1}$, George McGillivray ${ }^{1,2}$ and Joanne Kelley

${ }^{1}$ Mercy Hospital for Women, Melbourne, VIC, Australia and ${ }^{2}$ Royal Women's Hospital, Melbourne, VIC, Australia, ${ }^{3}$ Royal Children's Hospital, Melbourne, VIC, Australia

Background: Testing for RASopathy during pregnancy has been ordered relatively sporadically and protocols/laboratory accreditation for this testing have been lacking. Recent literature has provided new guidelines around when prenatal RASopathy testing is most appropriate: Stuurman et al. (2019) recommend RASopathy testing in fetuses with nuchal translucency $(\mathrm{NT}) \geq 3.5 \mathrm{~mm}$ plus another RASopathy-related ultrasound finding, and in fetuses with an isolated NT $\geq 5.0 \mathrm{~mm}$. Implementation of these guidelines in Australasian services has not been reported. Aim: To apply the guidelines outlined by Stuurman et al. (2019) to an appropriate patient cohort and report on the use of these guidelines at our service. Methods: Eligible patients were offered prenatal RASopathy testing from June 2019 to May 2021. Prenatal RASopathy testing consisted of next generation sequencing (NGS) of 18 genes through Mater Pathology in Queensland. Indications and outcomes are reported here. Results: Five fetuses had RASopathy testing. The most common indication was isolated NT $\geq 5.0 \mathrm{~mm}$. Of five tested, two fetuses had pathogenic variants (in RIT1 and PTPN11), one of which also had a variant of unknown significance (in PTPN11). Three fetuses had no variants detected. Conclusion: With the growing use of NGS in pregnancy, balancing the cost, scope, and timing of investigations has led to increasingly complex testing decisions for prenatal services. Clear testing guidelines help services offer appropriate and timely testing and enable discussions around all relevant genetic investigations early in the patient's journey. Women will benefit by having a pathway of investigations to come, should they wish to proceed. 